\title{
Identification of conductivity imperfections of small diameter by boundary measurements. Continuous dependence and computational reconstruction.
}

\author{
D.J. Cedio-Fengya*, S. Moskow ${ }^{\dagger}$ and M.S. Vogelius
}

\begin{abstract}
We derive an asymptotic formula for the electrostatic voltage potential in the presence of a finite number of diametrically small inhomogeneities with conductivity different from the background conductivity. We use this formula to establish continuous dependence estimates and to design an effective computational identification procedure.
\end{abstract}

1. Introduction

2. The electrostatic problem

3. An energy estimate

4. Some additional preliminary estimates

5. An asymptotic formula for the voltage potential

6. Properties of the polarization tensor

7. The continuous dependence of the inhomogeneities

8. Computational results.

9. References

\section{Introduction}

The non-destructive inspection technique known as electrical impedance imaging has recently received considerable attention in the mathematical- as well as in the engineering literature $[2,4,10,14,17]$. Using this technique one seeks to determine information about the internal conductivity- (or impedance) profile of an object based on boundary information about the applied steady-state currents and corresponding voltage potentials. The goal could well be to image an entirely unknown internal conductivity profile, but frequently it may be somewhat more limited in scope: a priori one has some knowledge of the overall form of the conductivity profile and one then seeks to determine very specific

\footnotetext{
*Department of Mathematics, Rutgers University, New Brunswick, NJ 08903, USA. Current address: Mathematics Department, James Madison University, Harrisonburg, VA 22807, USA

'Department of Mathematics, University of Minnesota, Minneapolis, MN 55455

${ }^{\ddagger}$ Department of Mathematics, Rutgers University, New Brunswick, NJ 08903, USA
} 
features. Examples of the latter type are found in connection with the identification of cracks $[12,15]$ and the identification of one or more inhomogeneities $[1,6,7,8]$. Following this line of investigation the purpose of the present paper is to design an efficient method to determine the location and size of diametrically small conductivity imperfections inside a conductor of known background conductivity. The imperfections (inhomogeneities) have constant conductivities. These conductivies may be known or unknown, depending on the application. Unlike the inhomogeneities treated in $[1,6,8]$ the ones considered here are of small size, and this shall allow for the design of a very effective identification procedure. The identification of small inhomogeneities has previously been analysed in [7], but whereas those inhomogeneities were either perfectly insulating (voids) or perfectly conducting (merely a dual) the ones considered here are just required to have a (finite) conductivity different from the background conductivity.

The fundamental step in the design of our identification procedure is the derivation of an asymptotic formula for the steady-state voltage potential for a conductor with a finite number of well separated, small inhomogeneities. In one particular version this asymptotic formula states that the voltage potential has the form

$$
\begin{gathered}
u(y)=\epsilon^{n} \Sigma_{i=1}^{m} \frac{\gamma\left(z_{i}\right)\left(\gamma\left(z_{i}\right)-k_{i}\right)}{k_{i}} \nabla_{x} N\left(z_{i}, y\right) \cdot M_{i} \nabla_{x} U\left(z_{i}\right) \\
+\int_{\partial \Omega} \psi(x) N(x, y) d \sigma_{x}+O\left(\epsilon^{n+\frac{1}{2}}\right) .
\end{gathered}
$$

Here $\Omega$ is the bounded $n$-dimensional domain occupied by the conductor, $m$ is the number of inhomogeneities, $z_{i}, 1 \leq i \leq m$, represents the "centers" of the inhomogeneities (which are not necessarily balls) and $\epsilon$ is the common order of magnitude of their "diameters". The function $\gamma$ is the background conductivity and $k_{i}$ is the (constant) conductivity of the $i^{t h}$ inhomogeneity. $N(x, y)$ is the Neumann function corresponding to the domain $\Omega, \psi$ is the applied boundary current, and $U$ is the voltage potential corresponding to the background conductivity $\gamma$. Indeed with this notation

$$
U(y)=\int_{\partial \Omega} \psi(x) N(x, y) d \sigma_{x}
$$

$M_{i}$ (a symmetric, positive definite $n \times n$ matrix) is a "polarization" tensor associated with the $i^{t h}$ inhomogeneity. Finally, the notation $O\left(\epsilon^{n+\frac{1}{2}}\right)$ signifies a term which goes to zero like $\epsilon^{n+\frac{1}{2}}$, uniformly in $y$ when $y$ is bounded away from the inhomogeneities. For our identification procedure we shall only make use of the asymptotic representation formula (1) for $y \in \partial \Omega$. Two special cases of the formula (1) corresponding to inhomogeneities of conductivity 0 and of conductivity $\infty$ were previously derived in [7]. The analysis leading to the general formula is considerably different from that used in those two special cases. The two special formulas may be recovered by letting $k_{i}$ tend to 0 and $\infty$ respectively in the general formula derived here. At this point it should be noted that the 
the "polarization" tensor $M_{i}$ in addition to the shape of the $i^{\text {th }}$ inhomogeneity also depends on the conductivity ratio $\gamma\left(z_{i}\right) / k_{i}$. The formula (1) asserts that the expression

$$
\begin{aligned}
& \epsilon^{n} \Sigma_{i=1}^{m} \frac{\gamma\left(z_{i}\right)\left(\gamma\left(z_{i}\right)-k_{i}\right)}{k_{i}} \nabla_{x} N\left(z_{i}, y\right) \cdot M_{i} \nabla_{x} U\left(z_{i}\right) \\
& \quad+\int_{\partial \Omega} \psi(x) N(x, y) d \sigma_{x}
\end{aligned}
$$

is a good approximation to the voltage potential for inhomogeneities that are well separated and for $\epsilon$ that are sufficiently small; however, numerical evidence strongly indicate that it is also a quite reasonable approximation even for inhomogeneities that are relatively close and for $\epsilon$ that are fairly large.

The asymptotic formula (1) and a slight variation thereof are used for two different purposes. (a): Given two different sets of inhomogeneities such a formula allows us estimate the difference in the location and relative size of the inhomogeneities in terms of the difference of the two corresponding boundary voltage potentials (the two prescribed currents are the same). This estimate is similar to one derived earlier for the case of inhomogeneities of extreme conductivity. (b): Such a formula suggests a very effective procedure to identify, for instance, the location and the size of a finite number of inhomogeneities based on knowledge of the boundary voltage potential. Namely, to select the parameters of an approximate expression like (2) so that it best fits the the (measured) boundary voltage potential. We construct a procedure to obtain a (nearly) best fit by employing a fairly straightforward least-squares approach, and we demonstrate by numerical examples the viability of this procedure. If the size and the location of the inhomogeities were known, then it is natural to try to use an approximate expression, such as $(2)$, to reconstruct the conductivities $k_{i}$. We are currently undertaking work to develop an effective procedure for this purpose.

\section{The electrostatic problem}

In the introduction we have briefly made reference to some of the ingredients of the electrostatic conductivity problem. In this section we shall provide more concise definitions. We suppose the conducting component occupies a bounded, smooth subdomain of $\Re^{n}, n=2$ or 3 . For simplicity we take $\partial \Omega$ to be $C^{\infty}$, but this assumption could be considerably weakened. Let $\gamma(\cdot)$ denote the smooth background conductivity, that is the conductivity in the absence of any inhomogeneities. We suppose that

$$
0<c_{0} \leq \gamma(x) \leq C_{0}<\infty, \quad x \in \Omega
$$

for some fixed constants $c_{0}$ and $C_{0}$. For simplicity, we assume that $\gamma$ is $C^{\infty}(\bar{\Omega})$, but this latter assumption could be considerably weakened. The function $\psi$ denotes the imposed boundary current. It suffices that $\psi \in H^{1 / 2}(\partial \Omega)$, with 
$\int_{\partial \Omega} \psi d \sigma=0$. The background voltage potential, $U$, is the solution to the boundary value problem

$$
\begin{aligned}
\nabla \cdot(\gamma(x) \nabla U) & =0 \text { in } \Omega, \\
\gamma \frac{\partial U}{\partial \nu} & =\psi \text { on } \partial \Omega .
\end{aligned}
$$

Here $\nu$ denotes the unit outward normal to the domain $\Omega$. Let $m$ denote the number of inhomogeneities and suppose that each inhomogeneity has the form $z_{i}+\epsilon B_{i}$, where $B_{i} \subset \Re^{n}$ is a bounded, smooth domain containing the origin. We assume that each $B_{i}$ is strictly star-shaped (meaning there exists $y_{0} \in B_{i}$ such that $\left(y-y_{0}\right) \cdot \nu>0$ for $y \in \partial B_{i}, \nu$ denoting the outer normal to $\left.B_{i}\right)$. For simplicity we assume that $B_{i}$ is a $C^{\infty}$ domain, but this assumption could be considerably weakened. The points $z_{i} \in \Omega, i=1, \ldots m$, determine the location of the inhomogeneities; we shall assume they satisfy

$$
\begin{gathered}
0<d_{0} \leq\left|z_{i}-z_{j}\right|, \quad \forall i \neq j \\
0<d_{0} \leq \operatorname{dist}\left(z_{i}, \partial \Omega\right), \quad \forall i
\end{gathered}
$$

We also assume that $\epsilon$, the common order of magnitude of the diameters of the inhomogeneities, is sufficiently small so that the inhomogeneities are disjoint and their distance to $\Re^{n} \backslash \Omega$ is larger than $d_{0} / 2$.

Let $\hat{\gamma}_{\epsilon}$ denote the conductivity profile in the presence of the small inhomogeneities. The function $\hat{\gamma}_{\epsilon}$ is equal to $\gamma$ except on the inhomogeneities; on the $i^{t h}$ inhomogeneity, $z_{i}+\epsilon B_{i}$, we have $\hat{\gamma}_{\epsilon}=k_{i}$, where $k_{i}, i=1 \ldots m$, is a set of positive constants. Let

$$
\omega_{\epsilon}=\bigcup_{i=1}^{m}\left(z_{i}+\epsilon B_{i}\right)
$$

denote the total collection of inhomogeneities. With this notation we have

$$
\hat{\gamma}_{\epsilon}(x)= \begin{cases}\gamma(x), & x \in \Omega \backslash \bar{\omega}_{\epsilon} \\ k_{i}, & x \in z_{i}+\epsilon B_{i}, i=1 \ldots m\end{cases}
$$

The voltage potential in the presence of the inhomogeneities is denoted $u_{\epsilon}(x)$. It is the solution to

$$
\begin{aligned}
\nabla \cdot\left(\hat{\gamma}_{\epsilon}(x) \nabla u_{\epsilon}\right) & =0 \quad \text { in } \Omega, \\
\hat{\gamma}_{\epsilon} \frac{\partial u_{\epsilon}}{\partial \nu} & =\psi \text { on } \partial \Omega .
\end{aligned}
$$

We normalize both $\mathrm{U}$ and $u_{\epsilon}$ by requiring that

$$
\int_{\partial \Omega} U d \sigma=0, \text { and } \int_{\partial \Omega} u_{\epsilon} d \sigma=0
$$


To ensure the validity of our continuous dependence results and to guarantee the success of our identification procedure it becomes necessary to assume that the following non-degeneracy conditions hold:

$$
\nabla_{x} U(x) \neq 0 \quad \forall x \in \Omega, \text { and } \gamma\left(z_{i}\right) \neq k_{i} \quad i=1, \ldots, m .
$$

These conditions are necessary and sufficient to guarantee that each of the terms

$$
\frac{\gamma\left(z_{i}\right)\left(\gamma\left(z_{i}\right)-k_{i}\right)}{k_{i}} \nabla_{x} N\left(z_{i}, y\right) \cdot M_{i} \nabla_{x} U\left(z_{i}\right)
$$

from the expression (2) is nontrivial. If this were not true then any continuous dependence result for the $i^{t h}$ inhomogeneity would depend on a higher order term in the asymptotic expansion of $u_{\epsilon}$, and it would not be as strong. Similarly the ability to effectively identify the $i^{t h}$ inhomogeneity would depend on finding the explicit form of this (higher order, nontrivial) term. As a result the identification would be considerably more complicated and not nearly as accurate.

In the computational identification experiments we present at the end of this paper, the inhomogeneities are assumed to be of the form $z_{i}+\epsilon \rho_{i} B$ or $z_{i}+\epsilon Q_{i} B$ (or even $z_{i}+\epsilon \rho_{i} Q_{i} B$ ) for a common, known domain $B$, but for unknown locations, $z_{i}$, dilatation parameters, $\epsilon \rho_{i}>0$, and rotations $Q_{i}$. The conductivities $k_{i}$ are also assumed to be known, and we seek to identify specific values of the unknown parameters associated with the inhomogeneities.

\section{$3 \quad$ An energy estimate}

We start the derivation of the asymptotic formula for $u_{\epsilon}$ with the following estimate of the $H^{1}(\Omega)$ norm of $U-u_{\epsilon}$.

Lemma 1 . There exists a constant $C$, independent of $\epsilon$, such that

$$
\int_{\Omega}\left(\left|\nabla\left(U-u_{\epsilon}\right)\right|^{2}+\left|U-u_{\epsilon}\right|^{2}\right) d x \leq C \epsilon^{n} .
$$

Proof . Since $\int_{\Omega}\left|U-u_{\epsilon}\right|^{2} d x \leq C\left(\int_{\Omega}\left|\nabla\left(U-u_{\epsilon}\right)\right|^{2} d x+\left|\int_{\partial \Omega}\left(U-u_{\epsilon}\right) d \sigma\right|^{2}\right)=$ $C \int_{\Omega}\left|\nabla\left(U-u_{\epsilon}\right)\right|^{2} d x$, it suffices to show that

$$
\int_{\Omega}\left|\nabla\left(U-u_{\epsilon}\right)\right|^{2} d x \leq C \epsilon^{n} .
$$

For $v \in H^{1}(\Omega)$, let $E_{\epsilon}(v)$ be the energy defined by

$$
E_{\epsilon}(v)=\frac{1}{2} \int_{\Omega} \hat{\gamma}_{\epsilon}|\nabla v|^{2} d x-\int_{\partial \Omega} \psi v d \sigma
$$

Then, since $\int_{\Omega} \gamma|\nabla U|^{2} d x=\int_{\partial \Omega} \psi U d \sigma$ and $\hat{\gamma}_{\epsilon}=\gamma$ on $\Omega \backslash \omega_{\epsilon}$,

$$
\begin{aligned}
E_{\epsilon}(U) & =\frac{1}{2} \int_{\Omega} \hat{\gamma}_{\epsilon}|\nabla U|^{2} d x-\int_{\partial \Omega} \psi U d \sigma \\
& =-\frac{1}{2} \int_{\Omega} \gamma|\nabla U|^{2} d x+\frac{1}{2} \int_{\omega_{\epsilon}}\left(\hat{\gamma}_{\epsilon}-\gamma\right)|\nabla U|^{2} d x .
\end{aligned}
$$


Expanding, integrating by parts, and using the formula for $E_{\epsilon}(U)$ given above, we see that

$$
\begin{aligned}
\int_{\Omega} \hat{\gamma}_{\epsilon}\left|\nabla\left(U-u_{\epsilon}\right)\right|^{2} d x & =\int_{\Omega} \hat{\gamma}_{\epsilon}|\nabla U|^{2} d x-2 \int_{\Omega} \hat{\gamma}_{\epsilon} \nabla u_{\epsilon} \nabla U d x+\int_{\Omega} \hat{\gamma}_{\epsilon}\left|\nabla u_{\epsilon}\right|^{2} d x \\
& =\int_{\Omega} \hat{\gamma}_{\epsilon}|\nabla U|^{2} d x-2 \int_{\partial \Omega} \psi U d \sigma+\int_{\Omega} \hat{\gamma}_{\epsilon}\left|\nabla u_{\epsilon}\right|^{2} d x \\
& =2 E_{\epsilon}(U)+\int_{\Omega} \hat{\gamma}_{\epsilon}\left|\nabla u_{\epsilon}\right|^{2} d x \\
& =-\int_{\Omega} \gamma|\nabla U|^{2} d x+\int_{\omega_{\epsilon}}\left(\hat{\gamma}_{\epsilon}-\gamma\right)|\nabla U|^{2} d x \\
& \quad+\int_{\Omega} \hat{\gamma}_{\epsilon}\left|\nabla u_{\epsilon}\right|^{2} d x
\end{aligned}
$$

We note that $\int_{\Omega} \hat{\gamma}_{\epsilon}\left|\nabla u_{\epsilon}\right|^{2} d x=\int_{\Omega} \gamma \nabla U \nabla u_{\epsilon} d x$ and $\int_{\Omega} \gamma|\nabla U|^{2} d x=\int_{\Omega} \hat{\gamma}_{\epsilon} \nabla u_{\epsilon} \nabla U d x$, which together yield

$$
\int_{\Omega} \hat{\gamma}_{\epsilon}\left|\nabla u_{\epsilon}\right|^{2} d x-\int_{\Omega} \gamma|\nabla U|^{2} d x=\int_{\omega_{\epsilon}}\left(\gamma-\hat{\gamma}_{\epsilon}\right) \nabla u_{\epsilon} \nabla U d x
$$

Substituting (9) into (8) and simplifying we obtain

$$
\begin{aligned}
\int_{\Omega} \hat{\gamma}_{\epsilon}\left|\nabla\left(U-u_{\epsilon}\right)\right|^{2} d x & =\int_{\omega_{\epsilon}}\left(\hat{\gamma}_{\epsilon}-\gamma\right)\left(\nabla U-\nabla u_{\epsilon}\right) \nabla U d x \\
& \leq\left(\int_{\omega_{\epsilon}}\left|\nabla\left(U-u_{\epsilon}\right)\right|^{2} d x\right)^{\frac{1}{2}}\left(\int_{\omega_{\epsilon}}\left(\hat{\gamma}_{\epsilon}-\gamma\right)^{2}|\nabla U|^{2} d x\right)^{\frac{1}{2}} .
\end{aligned}
$$

Thus, since $\hat{\gamma}_{\epsilon}$ is positive and uniformly bounded away from zero, and since $\gamma$ and $\hat{\gamma}_{\epsilon}$ are both uniformly bounded, it follows that

$$
\begin{aligned}
\int_{\Omega}\left|\nabla\left(U-u_{\epsilon}\right)\right|^{2} d x & \leq C \int_{\Omega} \hat{\gamma}_{\epsilon}\left|\nabla\left(U-u_{\epsilon}\right)\right|^{2} d x \\
& \leq C\left(\int_{\Omega}\left|\nabla\left(U-u_{\epsilon}\right)\right|^{2} d x\right)^{\frac{1}{2}}\left(\int_{\omega_{\epsilon}}|\nabla U|^{2} d x\right)^{\frac{1}{2}} .
\end{aligned}
$$

Combining this last estimate with the fact that $|\nabla U|$ is uniformly bounded on $\omega_{\epsilon}$ (due to elliptic regularity) we finally obtain

$$
\left(\int_{\Omega}\left|\nabla\left(U-u_{\epsilon}\right)\right|^{2} d x\right)^{\frac{1}{2}} \leq C\left(\int_{\omega_{\epsilon}}|\nabla U|^{2} d x\right)^{\frac{1}{2}} \leq C \epsilon^{\frac{n}{2}},
$$

from which the lemma immediately follows.

\section{Some additional preliminary estimates}

We begin by making the change of variables,

$$
y=x / \epsilon
$$


and restating the energy estimate from the last section in terms of $y$. Let

$$
\tilde{\Omega}=\left\{\frac{x}{\epsilon}: x \in \Omega\right\}
$$

be the corresponding rescaled domain. From Lemma 1 it follows that,

$$
\begin{gathered}
\left\|u_{\epsilon}(\epsilon y)-U(\epsilon y)\right\|_{L^{2}(\tilde{\Omega})} \leq C, \\
\left\|\nabla_{y}\left(u_{\epsilon}(\epsilon y)-U(\epsilon y)\right)\right\|_{L^{2}(\tilde{\Omega})} \leq C \epsilon,
\end{gathered}
$$

for some constant $C$, independent of $\epsilon$. To obtain a higher order estimate, we need to subtract another function from $u_{\epsilon}-U$.

For the remainder of this section we consider the case of a single inhomogeneity $\epsilon B$, which for simplicity we have assumed to be "centered" at the origin, with conductivity $k$. Let $v_{\epsilon}(y)$ denote the unique solution to

$$
\begin{gathered}
\Delta_{y} v_{\epsilon}=0 \quad \text { in } B, \quad \nabla_{y} \cdot \gamma(\epsilon y) \nabla_{y} v_{\epsilon}=0 \quad \text { in } \quad \tilde{\Omega} \backslash \bar{B} \\
v_{\epsilon}(y) \text { is continuous across } \partial B \\
\gamma(\epsilon y) \frac{\partial v_{\epsilon}^{+}}{\partial \nu_{y}}-k \frac{\partial v_{\epsilon}{ }^{-}}{\partial \nu_{y}}=-(\gamma(0)-k) \nabla_{x} U(0) \cdot \nu \text { on } \partial B \\
\gamma(\epsilon y) \frac{\partial v_{\epsilon}}{\partial \nu_{y}}=0 \quad \text { on } \partial \tilde{\Omega},
\end{gathered}
$$

with $\int_{\partial \tilde{\Omega}} v_{\epsilon} d \sigma=0$. The notation $\nu$ (or frequently $\nu_{y}$ ) is used for the outward unit normal to both $\tilde{\Omega}$ and $B$. The existence and uniqueness of $v_{\epsilon}$ is most easily established by variational means. Elliptic regularity estimates then guarantee that it is indeed a classical solution. Let $v(y)$ denote a solution to

$$
\begin{gathered}
\Delta_{y} v=0 \quad \text { in } B \quad, \quad \Delta_{y} v=0 \quad \text { in } \quad \Re^{n} \backslash \bar{B} \\
v \text { is continuous across } \partial B \\
\gamma(0) \frac{\partial v^{+}}{\partial \nu_{y}}-\underset{\lim ^{+} \nu_{y}}{ }{ }^{-}=-(\gamma(0)-k) \nabla_{x} U(0) \cdot \nu \text { on } \partial B \\
\lim _{|y| \rightarrow \infty} v(y)=0 .
\end{gathered}
$$

The existence of $v$ is most easily established by representing it as a single layer potential on $\partial B$ for a suitably chosen smooth density (that solves an associated boundary integral equation, cf. [5] or [11]). A single layer potential always vanishes at infinity for $n \geq 3$, for $n=2$ the vanishing at infinity of this particular representation relies on the fact that $\int_{\partial B} \nabla_{x} U(0) \cdot \nu_{y} d \sigma_{y}=0$. A maximum principle argument immediately gives that the solution to (13) is unique. We shall use the fact (cf. [5]) that the function $v$ exhibits the following behavior (decay) at infinity

$$
v(y)=\left\{\begin{array}{l}
O\left(|y|^{-1}\right), \quad n=2 \\
O\left(|y|^{2-n}\right), \quad n \geq 3
\end{array}\right.
$$


and

$$
\nabla_{y} v(y)= \begin{cases}O\left(|y|^{-2}\right), & n=2 \\ O\left(|y|^{1-n}\right), & n \geq 3\end{cases}
$$

This decay ensures that for any $n$,

$$
\|v\|_{L^{2}(\tilde{\Omega})} \leq C / \epsilon^{1 / 2}
$$

We now proceed with the analysis which eventually leads to an estimate for $\nabla_{y}\left(u_{\epsilon}(\epsilon y)-U(\epsilon y)-\epsilon v(y)\right)$ (Theorem 1) an estimate that plays a crucial role in the derivation of our representation formula. The proof of Theorem 1 we provide holds for two and three dimensions, but with minimal changes it could be extended to higher dimensions as well. We shall later point out the single place where the fact that the dimension is either two or three is used. We first prove the following lemma concerning $\nabla_{y}\left(u_{\epsilon}(\epsilon y)-U(\epsilon y)-\epsilon v_{\epsilon}(y)\right)$

Lemma 2. There exists a constant $C$, independent of $\epsilon$, such that

$$
\left\|\nabla_{y}\left(u_{\epsilon}(\epsilon y)-U(\epsilon y)-\epsilon v_{\epsilon}(y)\right)\right\|_{L^{2}(\tilde{\Omega})} \leq C \epsilon^{2} .
$$

Proof. Define

$$
z_{\epsilon}(y)=u_{\epsilon}(\epsilon y)-U(\epsilon y)-\epsilon v_{\epsilon}(y)-c_{\epsilon}
$$

where the constant $c_{\epsilon}$ is chosen so that

$$
\int_{\partial B} z_{\epsilon} d \sigma_{y}=0
$$

Using the equations (3), (6) and (12) we compute that $z_{\epsilon}$ solves

$$
\begin{gathered}
\Delta_{y} z_{\epsilon}=-\Delta_{y} U(\epsilon y) \quad \text { in } B, \quad \nabla_{y} \cdot \gamma(\epsilon y) \nabla_{y} z_{\epsilon}=0 \quad \text { in } \tilde{\Omega} \backslash \bar{B} \\
z_{\epsilon} \text { is continuous across } \partial B, \text { and } \gamma(\epsilon y) \frac{\partial z_{\epsilon}}{\partial \nu_{y}}=0 \quad \text { on } \partial \tilde{\Omega},
\end{gathered}
$$

along with the jump condition

$$
\gamma(\epsilon y) \frac{\partial z_{\epsilon}{ }^{+}}{\partial \nu_{y}}-k{\frac{\partial z_{\epsilon}}{\partial \nu_{y}}}^{-}=\epsilon(\gamma(0)-k) \nabla_{x} U(0) \cdot \nu_{y}-(\gamma(\epsilon y)-k) \nabla_{y} U(\epsilon y) \cdot \nu_{y}
$$

on $\partial B$. Now consider the quantity

$$
\int_{\tilde{\Omega}} \hat{\gamma}_{\epsilon}(\epsilon y) \nabla_{y} z_{\epsilon} \cdot \nabla_{y} z_{\epsilon} d y=\int_{\tilde{\Omega} \backslash \bar{B}} \gamma(\epsilon y) \nabla_{y} z_{\epsilon} \cdot \nabla_{y} z_{\epsilon} d y+\int_{B} k \nabla_{y} z_{\epsilon} \cdot \nabla_{y} z_{\epsilon} d y
$$

Integrating each term on the right by parts

$$
\begin{aligned}
\int_{\tilde{\Omega}} \hat{\gamma}_{\epsilon}(\epsilon y) \nabla_{y} z_{\epsilon} \cdot \nabla_{y} z_{\epsilon} d y \\
=\int_{\partial B}\left[k \frac{\partial z_{\epsilon}-}{\partial \nu_{y}}-\gamma(\epsilon y) \frac{\partial z_{\epsilon}}{\partial \nu_{y}}\right] z_{\epsilon} d \sigma_{y}+\int_{B} k \Delta_{y} U(\epsilon y) z_{\epsilon} d y \\
=\int_{\partial B} \epsilon\left[(\gamma(\epsilon y)-k) \nabla_{x} U(\epsilon y)-(\gamma(0)-k) \nabla_{x} U(0)\right] \cdot \nu_{y} z_{\epsilon} d \sigma_{y} \\
\quad+\epsilon^{2} \int_{B} k \Delta_{x} U(\epsilon y) z_{\epsilon} d y
\end{aligned}
$$


Using Taylor's theorem, the smoothness of $\gamma$, and the fact that $U$ and all of its first and second derivatives are uniformly bounded on $\epsilon B$, we get that

$$
(\gamma(\epsilon y)-k) \nabla_{x} U(\epsilon y)-(\gamma(0)-k) \nabla_{x} U(0)
$$

is $O(\epsilon)$ in $L^{\infty}(\partial B)$ (hence $O(\epsilon)$ in $H^{-1 / 2}(\partial B)$ ) and that $\triangle_{x} U(\epsilon y)$ is bounded in $L^{2}(B)$. Therefore, by the trace theorem

$$
\left\|\nabla_{y} z_{\epsilon}\right\|_{L^{2}(\tilde{\Omega})}^{2} \leq C \epsilon^{2}\left\|z_{\epsilon}\right\|_{H^{1 / 2}(\partial B)}+C \epsilon^{2}\left\|z_{\epsilon}\right\|_{L^{2}(B)} \leq C \epsilon^{2}\left\|z_{\epsilon}\right\|_{H^{1}(B)} .
$$

Note that in defining $z_{\epsilon}$ we have subtracted the constant $c_{\epsilon}$ so that we can apply Poincaire's inequality on $B$. In doing so we obtain

$$
\left\|\nabla_{y} z_{\epsilon}\right\|_{L^{2}(\tilde{\Omega})}^{2} \leq C \epsilon^{2}\left\|\nabla_{y} z_{\epsilon}\right\|_{L^{2}(B)} \leq C \epsilon^{2}\left\|\nabla_{y} z_{\epsilon}\right\|_{L^{2}(\tilde{\Omega})}
$$

which, upon division, implies the lemma.

The following corollaries now follow from Poincare's inequality and a change of variables.

Corollary 1. There exists a constant $C$, independent of $\epsilon$, such that

$$
\left\|u_{\epsilon}(\epsilon y)-U(\epsilon y)-\epsilon v_{\epsilon}(y)\right\|_{L^{2}(\tilde{\Omega})} \leq C \epsilon .
$$

Corollary 2. There exists a constant $C$, independent of $\epsilon$, such that

$$
\left\|u_{\epsilon}(x)-U(x)-\epsilon v_{\epsilon}(x / \epsilon)\right\|_{H^{1}(\Omega)} \leq C \epsilon^{n / 2+1} .
$$

We shall need a somewhat special Poincare-type inequality:

Lemma 3 . Let $f \in L^{\infty}(\partial B)$ with $0<\alpha \leq f \leq \beta$. Then there exists a constant C such that

$$
\left(\int_{B}|u|^{2} d y\right)^{\frac{1}{2}} \leq C\left(\left(\int_{B}\left|\nabla_{y} u\right|^{2} d y\right)^{\frac{1}{2}}+\left|\int_{\partial B} f u d \sigma_{y}\right|\right) \quad \forall u \in H^{1}(B) .
$$

The constant $C$ depends on the constants $\alpha$ and $\beta$, but is otherwise independent of $f$.

Proof. We shall prove the above by contradiction. Suppose (17) is not true, then for all $m, \exists u_{m}$ in $H^{1}(B)$ and $0<\alpha \leq f_{m} \leq \beta$ such that

$$
\left\|u_{m}\right\|_{L^{2}(B)}>m\left(\left\|\nabla u_{m}\right\|_{L^{2}(B)}+\left|\int_{\partial B} u_{m} f_{m} d \sigma_{y}\right|\right) .
$$

We may normalize by taking $\left\|u_{m}\right\|_{L^{2}(B)}=1 \quad \forall m$. Then $\left\|\nabla u_{m}\right\|_{L^{2}(B)}<\frac{1}{m}$. By compactness, there exists a subsequences, $u_{m}, f_{m}$ (with common index, $m \rightarrow \infty$ ) 
such that $u_{m} \rightarrow u$ in $H^{1}(B), u_{m} \rightarrow u$ in $L^{2}(B),\left.\left.u_{m}\right|_{\partial B} \rightarrow u\right|_{\partial B}$ in $L^{2}(\partial B)$ and $f_{m} \rightarrow f$ in $L^{2}(\partial B), 0<\alpha \leq f \leq \beta$. Passing to the limit we have

$$
0 \leq\|\nabla u\|_{L^{2}(B)} \leq \liminf \left\|\nabla u_{m}\right\|_{L^{2}(B)} \leq \lim _{m \rightarrow \infty} \frac{1}{m}=0 .
$$

This implies $u \equiv$ constant in $B$. In addition,

$$
\left|\int_{\partial B} u f d \sigma_{y}\right|=\lim \left|\int_{\partial B} u_{m} f_{m} d \sigma_{y}\right| \leq \lim _{m \rightarrow \infty} \frac{1}{m}=0 .
$$

Now, since $\left|\int_{\partial B} u f d \sigma_{y}\right|=0$ and $\int_{\partial B} f d \sigma_{y} \neq 0$, it follows that $u=0$. However, if $u=0$ then $\|u\|_{L^{2}(B)}=0$ which contradicts the fact that $u_{m} \rightarrow u$ in $L^{2}(B)$ and $\left\|u_{m}\right\|_{L^{2}(B)}=1$.

We now come to the only place in our analysis where we shall use the fact that $n$ is either 2 or 3 .

Lemma 4. Suppose that $n=2$ or 3 and suppose $B$ is strictly star-shaped with respect to the origin. Then there exist constants $0<\epsilon_{0}$ and $C$ such that

$$
\left\|v_{\epsilon}\right\|_{H^{1}(B)} \leq C \quad, \quad 0<\epsilon<\epsilon_{0}
$$

Proof . A combination of Lemma 2 and the estimate (11) immediately yields $\left\|\nabla_{y} v_{\epsilon}\right\|_{L^{2}(B)} \leq\left\|\nabla_{y} v_{\epsilon}\right\|_{L^{2}(\tilde{\Omega})} \leq C$. From Lemma 3 we know that

$$
\left(\int_{B}\left|v_{\epsilon}\right|^{2} d y\right)^{\frac{1}{2}} \leq C\left(\left(\int_{B}\left|\nabla_{y} v_{\epsilon}\right|^{2} d y\right)^{\frac{1}{2}}+\left|\int_{\partial B} f_{\epsilon} v_{\epsilon} d \sigma_{y}\right|\right)
$$

provided $0<\alpha \leq f_{\epsilon} \leq \beta$. In order to prove this lemma it thus suffices to show that

$$
\left|\int_{\partial B} f_{\epsilon} v_{\epsilon} d \sigma_{y}\right|<C
$$

for some appropriate choice of $0<\alpha \leq f_{\epsilon} \leq \beta$.

Let $N(x)$ be the Neumann function in $\Omega$ corresponding to a Dirac mass at the origin and to coefficient $\gamma$. That is, $N$ is the solution to

$$
\begin{aligned}
-\nabla_{x} \gamma(x) \nabla_{x} N & =\delta_{0} \quad \text { in } \quad \Omega \\
\gamma(x) \frac{\partial N}{\partial \nu_{x}} & =-\frac{1}{|\partial \Omega|} \quad \text { on } \quad \partial \Omega .
\end{aligned}
$$

For $n=2$ we can express $N$ as the sum of a logarithm and a smoother function [7]. More precisely

$$
N(x)=\Phi_{2}(x)+R_{2}(x)=-\frac{1}{2 \pi \gamma(0)} \log |x|+R_{2}(x) \quad(n=2),
$$


where $R_{2}(x) \in W^{2, p}(\Omega)$ for any $1 \leq p<2$ and solves

$$
\begin{aligned}
\nabla_{x} \cdot \gamma(x) \nabla_{x} R_{2}(x) & =\frac{1}{2 \pi \gamma(0)} \frac{\nabla_{x} \gamma(x) \cdot x}{|x|^{2}} \quad x \in \Omega, \\
\gamma(x) \frac{\partial R_{2}}{\partial \nu_{x}}(x) & =-\frac{1}{|\partial \Omega|}+\frac{\gamma(x)}{2 \pi \gamma(0)} \frac{x \cdot \nu_{x}}{|x|^{2}} \quad x \in \partial \Omega .
\end{aligned}
$$

For $n=3$ we can similarly express $N$ as the sum of $\frac{1}{4 \pi \gamma(0)}|x|^{-1}$ and a remainder in $W^{2, p}(\Omega), 1 \leq p<3 / 2$, but we shall need a more refined expansion, namely

$$
N(x)=\Phi_{3}(x)+R_{3}(x)=\frac{1}{4 \pi \gamma(0)}|x|^{-1}-\frac{\nabla \gamma(0) \cdot x}{8 \pi[\gamma(0)]^{2}}|x|^{-1}+R_{3}(x) \quad(n=3),
$$

where the remainder, $R_{3}$, is in $W^{2, p}(\Omega)$ for any $1 \leq p<3$ and solves

$$
\begin{gathered}
\nabla_{x} \cdot \gamma(x) \nabla_{x} R_{3}(x)=A \cdot\left[|x|^{2} \nabla \gamma(x)-(\nabla \gamma(x) \cdot x) x+2(\gamma(0)-\gamma(x)) x\right]|x|^{-3} \\
\quad+\frac{1}{4 \pi \gamma(0)}[\nabla \gamma(x)-\nabla \gamma(0)] \cdot x|x|^{-3} \quad x \in \Omega, \quad(22) \quad x \in \partial \Omega \\
\gamma(x) \frac{\partial R_{3}}{\partial \nu_{x}}(x)=-\frac{1}{|\partial \Omega|}-\gamma(x) \frac{\partial \Phi_{3}}{\partial \nu_{x}} \quad x \in \partial \Omega
\end{gathered}
$$

Here the constant vector $A$ is given by $A=\frac{1}{8 \pi[\gamma(0)]^{2}} \nabla \gamma(0)$. From the way we have written the right hand side of (22) it follows immediately that it is of order $|x|^{-1}$ at the origin, and therefore in $L^{p}(\Omega)$, for any $1 \leq p<3$. It is based on this fact and elliptic regularity that we conclude $R_{3}$ is in $W^{2, p}, 1 \leq p<3$. Since in particular $R_{n} \in H^{1}(\Omega)$ (and $n=2,3$ ) we get

$$
\epsilon^{2(n-2)} \int_{B}\left|\nabla_{y} R_{n}(\epsilon y)\right|^{2} d y=\epsilon^{n-2} \int_{\epsilon B}\left|\nabla_{x} R_{n}(x)\right|^{2} d x \leq C .
$$

The fact that $R_{n}$ is in $W^{2, p}(\Omega)$ for any $p<n(n=2,3)$ implies that $R_{n}$ is continuous (and thus uniformly bounded) on $\bar{\Omega}$. Thus

$$
\epsilon^{2(n-2)} \int_{B}\left|R_{n}(\epsilon y)\right|^{2} d y \leq C \epsilon^{2(n-2)} \leq C
$$

From a combination of these two inequality we conclude that $\epsilon^{n-2} R_{n}(\epsilon y)$ is bounded in $H^{1}(B)$ and therefore by the trace theorem

$$
\left\|\epsilon^{n-2} R_{n}(\epsilon y)\right\|_{H^{1 / 2}(\partial B)} \leq C \quad(n=2,3) .
$$

We introduce the function

$$
N_{\epsilon}(y)=\left\{\begin{array}{ll}
N(\epsilon y)+\frac{\log \epsilon}{2 \pi \gamma(0)} & (n=2) \\
\epsilon N(\epsilon y) & (n=3)
\end{array} .\right.
$$


Based on the formulas given above for $N$ and the estimate (24) for $\epsilon^{n-2} R_{n}(\epsilon y)$ it follows immediately that

$$
\left\|N_{\epsilon}\right\|_{H^{1 / 2}(\partial B)} \leq C \quad(n=2,3) .
$$

In establishing (19) we shall need the normal derivative of $N_{\epsilon}$ on $\partial B$. A simple calculation yields

$$
\nabla_{y} N_{\epsilon}=\frac{-1}{2 \pi \gamma(0)} \frac{y}{|y|^{2}}+\nabla_{y} R_{2}(\epsilon y) \quad(n=2)
$$

and

$$
\begin{gathered}
\nabla_{y} N_{\epsilon}=-\frac{1}{4 \pi \gamma(0)}|y|^{-3}\left(y+\frac{\epsilon}{2 \gamma(0)} \nabla \gamma(0) \cdot y^{\perp} y^{\perp}\right) \\
+\epsilon \nabla_{y} R_{3}(\epsilon y) \quad(n=3) .
\end{gathered}
$$

Thus, on $\partial B$

$$
\gamma(\epsilon y) \frac{\partial N_{\epsilon}}{\partial \nu_{y}}=-f_{n, \epsilon}+\epsilon^{n-2} \gamma(\epsilon y) \frac{\partial R_{n}(\epsilon y)}{\partial \nu_{y}},
$$

where $f_{n, \epsilon}, n=2,3$, is given by

$$
f_{2, \epsilon}(y)=\frac{\gamma(\epsilon y)}{2 \pi \gamma(0)}|y|^{-2} y \cdot \nu_{y}
$$

and

$$
f_{3, \epsilon}(y)=\frac{\gamma(\epsilon y)}{4 \pi \gamma(0)}|y|^{-3}\left(y+\frac{\epsilon}{2 \gamma(0)} \nabla \gamma(0) \cdot y^{\perp} y^{\perp}\right) \cdot \nu_{y} .
$$

The positivity of $\gamma$ and the condition that $B$ is strictly star shaped with respect to the origin guarantee that there exist constants $0<\epsilon_{0}, \alpha$ and $\beta$ such that $0<\alpha \leq f_{n, \epsilon}(y) \leq \beta$ for $0<\epsilon<\epsilon_{0}$. Rearranging (27) we obtain

$$
\left|\int_{\partial B} f_{n, \epsilon} v_{\epsilon} d \sigma_{y}\right| \leq\left|\int_{\partial B} \gamma(\epsilon y) \frac{\partial N_{\epsilon}}{\partial \nu_{y}} v_{\epsilon} d \sigma_{y}\right|+\epsilon^{n-2}\left|\int_{\partial B} \gamma(\epsilon y) \nabla_{y} R_{n}(\epsilon y) \cdot \nu_{y} v_{\epsilon} d \sigma_{y}\right| \text {. }
$$

Integrating by parts, and using equations (12) and (20) we see that

$$
\begin{aligned}
\int_{\partial B} \gamma(\epsilon y) \frac{\partial N_{\epsilon}}{\partial \nu_{y}} v_{\epsilon} d \sigma_{y} & =-\int_{\tilde{\Omega} \backslash \bar{B}} \gamma(\epsilon y) \nabla_{y} N_{\epsilon} \nabla_{y} v_{\epsilon} d y \\
& =\int_{\partial B} \gamma(\epsilon y) \frac{\partial v_{\epsilon}}{\partial \nu_{y}} N_{\epsilon} d \sigma_{y}
\end{aligned}
$$

and therefore

$$
\begin{aligned}
& \left|\int_{\partial B} f_{n, \epsilon} v_{\epsilon} d \sigma_{y}\right| \\
& \quad \leq\left|\int_{\partial B} \gamma(\epsilon y) \frac{\partial v_{\epsilon}}{\partial \nu_{y}}{ }^{+} N_{\epsilon} d \sigma_{y}\right|+\epsilon^{n-2}\left|\int_{\partial B} \gamma(\epsilon y) \nabla_{y} R_{n}(\epsilon y) \cdot \nu_{y} v_{\epsilon} d \sigma_{y}\right| .
\end{aligned}
$$


The first term on the right-hand side is bounded by

$$
\begin{aligned}
\left|\int_{\partial B} \gamma(\epsilon y) \frac{\partial v_{\epsilon}}{\partial \nu_{y}} N_{\epsilon} d \sigma_{y}\right| & \leq C\left\|\gamma(\epsilon y) \frac{\partial v_{\epsilon}}{\partial \nu_{y}}\right\|_{H^{-1 / 2}(\partial B)}\left\|N_{\epsilon}\right\|_{H^{1 / 2}(\partial B)} \\
& \leq C\left\|\gamma(\epsilon y) \frac{\partial v_{\epsilon}}{\partial \nu_{y}}\right\|_{H^{-1 / 2}(\partial B)},
\end{aligned}
$$

since $N_{\epsilon}$ is bounded in $H^{1 / 2}(\partial B)$, according to (26). Furthermore, we have that

$$
\gamma(\epsilon y) \frac{\partial v_{\epsilon}}{\partial \nu_{y}}=-(\gamma(0)-k) \nabla_{x} U(0) \cdot \nu_{y}+k{\frac{\partial v_{\epsilon}}{\partial \nu_{y}}}^{-}
$$

is bounded in $H^{-1 / 2}(\partial B)$ independently of $\epsilon$. To see the latter, let $\chi$ be any function in $H^{1 / 2}(\partial B)$ and extend $\chi$ into $B$ so that

$$
\|\chi\|_{H^{1}(B)} \leq C\|\chi\|_{H^{1 / 2}(\partial B)} \text {. }
$$

Then,

$$
\begin{aligned}
\int_{\partial B} k \frac{\partial v_{\epsilon}}{\partial \nu_{y}} \chi d \sigma_{y} & =\int_{B} k \nabla_{y} v_{\epsilon} \nabla_{y} \chi d y \\
& \leq C\left\|\nabla_{y} v_{\epsilon}\right\|_{L^{2}(B)}\|\chi\|_{H^{1 / 2}(\partial B)}
\end{aligned}
$$

Hence

$$
\left\|k \frac{\partial v_{\epsilon}}{\partial \nu_{y}}\right\|_{H^{-1 / 2}(\partial B)} \leq C\left\|\nabla_{y} v_{\epsilon}\right\|_{L^{2}(B)}
$$

the right hand side of which, as pointed out at the beginning of this proof, is bounded independently of $\epsilon$. It now immediately follows that

$$
\left\|\gamma(\epsilon y) \frac{\partial v_{\epsilon}}{\partial \nu_{y}}\right\|_{H^{-1 / 2}(\partial B)} \leq C
$$

as stated previously. The Estimates (29) and (31) in combination give that the first term on the right hand side of (28) is bounded independently of $\epsilon$, i.e.,

$$
\left|\int_{\partial B} \gamma(\epsilon y){\frac{\partial v_{\epsilon}}{\partial \nu_{y}}}^{+} N_{\epsilon} d \sigma_{y}\right| \leq C .
$$

We now seek a bound for the second term on the right-hand side of (28). Integrating twice by parts, and using the fact that $\triangle_{y} v_{\epsilon}=0$ in $B$ we get

$$
\begin{aligned}
\int_{\partial B} & \gamma(\epsilon y) \nabla_{y} R_{n}(\epsilon y) \cdot \nu_{y} v_{\epsilon} d \sigma_{y} \\
= & \int_{B} \gamma(\epsilon y) \nabla_{y} R_{n}(\epsilon y) \cdot \nabla_{y} v_{\epsilon} d y+\int_{B} \nabla_{y} \cdot \gamma(\epsilon y) \nabla_{y} R(\epsilon y) v_{\epsilon} d y \\
= & -\epsilon \int_{B} R_{n}(\epsilon y) \nabla_{x} \gamma(\epsilon y) \cdot \nabla_{y} v_{\epsilon} d y+\int_{\partial B} R_{n}(\epsilon y) \gamma(\epsilon y) \frac{\partial v_{\epsilon}}{\partial \nu_{y}} d \sigma_{y} \\
& \quad+\int_{B} \nabla_{y} \cdot \gamma(\epsilon y) \nabla_{y} R_{n}(\epsilon y) v_{\epsilon} d y
\end{aligned}
$$


From the equations (21) and (22) for $R_{n}$ it follows that

$$
\nabla_{y} \cdot \gamma(\epsilon y) \nabla_{y} R_{n}(\epsilon y)=\epsilon F_{n, \epsilon}(y)
$$

where

$$
\left\|F_{n, \epsilon}\right\|_{L^{p}(B)} \leq C_{p} \quad \text { for any } p<n,
$$

(independently of $\epsilon$ ). Based on (33) we may thus estimate

$$
\begin{gathered}
\left|\int_{\partial B} \gamma(\epsilon y) \nabla_{y} R_{n}(\epsilon y) \cdot \nu_{y} v_{\epsilon} d \sigma_{y}\right| \leq \epsilon\left\|R_{n} \nabla_{x} \gamma(\epsilon y)\right\|_{L^{2}(B)}\left\|\nabla_{y} v_{\epsilon}\right\|_{L^{2}(B)} \\
+\left\|R_{n} \gamma(\epsilon y)\right\|_{H^{1 / 2}(\partial B)}\left\|\frac{\partial v_{\epsilon}}{\partial \nu_{y}}\right\|_{H^{-1 / 2}(\partial B)} \\
+C_{q} \epsilon\left\|v_{\epsilon}\right\|_{L^{q}(B)},
\end{gathered}
$$

for any $q>\frac{n}{n-1}$ (the dual of $\left.p<n\right)$. Since we have already verified that $R_{n}(\epsilon y)$ is uniformly bounded on $B$ (independently of $\epsilon$ ) and that the three quantities $\left\|\nabla_{y} v_{\epsilon}\right\|_{L^{2}(B)},\left\|\epsilon^{n-2} R_{n}(\epsilon y)\right\|_{H^{1 / 2}(\partial B)}$ and $\left\|\frac{\partial v_{\epsilon}}{\partial \nu_{y}}{ }^{-}\right\|_{H^{-1 / 2}(\partial B)}$ are bounded it now follows that

$$
\epsilon^{n-2}\left|\int_{\partial B} \gamma(\epsilon y) \nabla_{y} R(\epsilon y) \cdot \nu_{y} v_{\epsilon} d \sigma_{y}\right| \leq C_{q}\left(1+\epsilon^{n-1}\left\|v_{\epsilon}\right\|_{L^{q}(B)}\right),
$$

for any $q>\frac{n}{n-1}$. Recall that $v_{\epsilon}$ integrates to zero on $\partial \tilde{\Omega}$. Since the gradient of $v_{\epsilon}$ is bounded in $L^{2}(\tilde{\Omega})$, a rescaling of Poincaire's inequality gives $\left\|v_{\epsilon}\right\|_{H^{1}(\tilde{\Omega})} \leq C / \epsilon$, which then by Sobolev's imbedding theorem implies that

$$
\left\|v_{\epsilon}\right\|_{L^{q}(B)} \leq C_{q}\left\|v_{\epsilon}\right\|_{H^{1}(B)} \leq C_{q} / \epsilon
$$

for any $q<\frac{2 n}{n-2}(q<\infty$ for $n=2)$. Hence, by selecting any $\frac{n}{n-1}<q<\frac{2 n}{n-2}$

$$
\epsilon^{n-2}\left|\int_{\partial B} \gamma(\epsilon y) \nabla_{y} R_{n}(\epsilon y) \cdot \nu_{y} v_{\epsilon} d \sigma_{y}\right| \leq C\left(1+\epsilon^{n-2}\right) \leq C
$$

This gives an $\epsilon$-independent bound for the second term on the right hand side of (28), and thus completes the proof of Lemma 4.

We are now in a position to establish the estimate which is crucial for the derivation of our representation formula. This estimate involves the function $v$, the solution to the problem (13). Since the previous lemma, which was only verified for dimensions 2 and 3 , is used in the proof, the same dimensional restriction will be maintained.

Theorem 1. Suppose $n=2$ or 3 and suppose $B$ is strictly star-shaped with respect to the origin. Then there exists a constant $C$, independent of $\epsilon$, such that

$$
\left\|\nabla_{y}\left(u_{\epsilon}(\epsilon y)-U(\epsilon y)-\epsilon v(y)\right)\right\|_{L^{2}(\tilde{\Omega})} \leq C \epsilon^{3 / 2} .
$$


Proof. It is clearly sufficient to prove the desired estimate for $\epsilon$ small, e.g., for $0<\epsilon<\epsilon_{0}$, with $\epsilon_{0}$ being the constant from Lemma 4. From Lemma 2 it follows that it suffices to show that

$$
\left\|\nabla_{y}\left(v_{\epsilon}-v\right)\right\|_{L^{2}(\tilde{\Omega})} \leq C \epsilon^{1 / 2} \quad 0<\epsilon<\epsilon_{0} .
$$

Now define

$$
w_{\epsilon}=v_{\epsilon}-v-c_{\epsilon}
$$

where $c_{\epsilon}$ is the constant chosen so that $\int_{\partial \tilde{\Omega}} w_{\epsilon} d \sigma_{y}=0$. Then $w_{\epsilon}$ solves

$$
\begin{gathered}
\Delta_{y} w_{\epsilon}=0 \quad \text { in } B, \quad \nabla_{y} \cdot \gamma(\epsilon y) \nabla_{y} w_{\epsilon}=-\epsilon \nabla_{x} \gamma(\epsilon y) \cdot \nabla_{y} v \quad \text { in } \quad \tilde{\Omega} \backslash \bar{B} \\
\gamma(\epsilon y) \frac{\partial w_{\epsilon}{ }^{+}}{\partial \nu_{y}}-k \frac{\partial w_{\epsilon}{ }^{-}}{\partial \nu_{y}}=(\gamma(0)-\gamma(\epsilon y)) \frac{\partial v^{+}}{\partial \nu_{y}} \quad \text { on continuous across } \partial B \\
\gamma(\epsilon y) \frac{\partial w_{\epsilon}}{\partial \nu_{y}}=-\gamma(\epsilon y) \frac{\partial v}{\partial \nu_{y}} \quad \text { on } \partial \tilde{\Omega} .
\end{gathered}
$$

For future reference we note that the condition $\int_{\partial \tilde{\Omega}} v_{\epsilon}=0$ combined with the decay of $v$ guarantee that the constants, $c_{\epsilon}$, are uniformly bounded (indeed they are bounded by $C \epsilon$ ). Consider the following integral identity

$$
\int_{\tilde{\Omega}} \hat{\gamma}_{\epsilon}(\epsilon y) \nabla_{y} w_{\epsilon} \cdot \nabla_{y} w_{\epsilon} d y=\int_{\tilde{\Omega} \backslash B} \gamma(\epsilon y) \nabla_{y} w_{\epsilon} \cdot \nabla_{y} w_{\epsilon} d y+\int_{B} k \nabla_{y} w_{\epsilon} \cdot \nabla_{y} w_{\epsilon} d y
$$

where $\hat{\gamma}$ is as defined previously. Integrating each term on the right hand side by parts, and using the equations for $w_{\epsilon}$, we obtain

$$
\begin{aligned}
\int_{\tilde{\Omega}} \hat{\gamma}_{\epsilon}(\epsilon y) \nabla_{y} w_{\epsilon} \cdot \nabla_{y} w_{\epsilon} d y= & \int_{\tilde{\Omega} \backslash \bar{B}} \epsilon \nabla_{x} \gamma(\epsilon y) \cdot \nabla_{y} v w_{\epsilon} d y-\int_{\partial \tilde{\Omega}} \gamma(\epsilon y) \frac{\partial v}{\partial \nu_{y}} w_{\epsilon} d \sigma_{y} \\
& +\int_{\partial B}(\gamma(\epsilon y)-\gamma(0)) \frac{\partial v^{+}}{\partial \nu_{y}} w_{\epsilon} d \sigma_{y}
\end{aligned}
$$

Continued integration by parts of the first term on the right hand side yields

$$
\begin{aligned}
& \int_{\tilde{\Omega}} \hat{\gamma}(\epsilon y) \nabla_{y} w_{\epsilon} \cdot \nabla_{y} w_{\epsilon} d y \\
&=-\epsilon^{2} \int_{\tilde{\Omega} \backslash \bar{B}}\left(\Delta_{x} \gamma(\epsilon y)\right) v w_{\epsilon} d y-\epsilon \int_{\tilde{\Omega} \backslash \bar{B}}\left(\nabla_{x} \gamma(\epsilon y)\right) v \cdot \nabla_{y} w_{\epsilon} d y \\
&+\int_{\partial \tilde{\Omega}} \epsilon\left(\nabla_{x} \gamma(\epsilon y) \cdot \nu_{y}\right) v w_{\epsilon} d \sigma_{y}-\int_{\partial B} \epsilon\left(\nabla_{x} \gamma(\epsilon y) \cdot \nu_{y}\right) v w_{\epsilon} d \sigma_{y} \\
&-\int_{\partial \tilde{\Omega}} \gamma(\epsilon y) \frac{\partial v}{\partial \nu_{y}} w_{\epsilon} d \sigma_{y}+\int_{\partial B}(\gamma(\epsilon y)-\gamma(0)) \frac{\partial v}{\partial \nu_{y}} w_{\epsilon} d \sigma_{y}
\end{aligned}
$$

We shall examine each term on the right hand side of (35) separately. The first term is bounded by

$$
\epsilon^{2}\left|\int_{\tilde{\Omega} \backslash \bar{B}}\left(\Delta_{x} \gamma(\epsilon y)\right) v w_{\epsilon} d y\right| \leq C \epsilon^{2}\|v\|_{L^{2}(\tilde{\Omega} \backslash \bar{B})}\left\|w_{\epsilon}\right\|_{L^{2}(\tilde{\Omega} \backslash \bar{B})}
$$


Application of a rescaled Poincaire's inequality and (16) now yields

$$
\begin{aligned}
\epsilon^{2}\left|\int_{\tilde{\Omega} \backslash \bar{B}}\left(\Delta_{x} \gamma(\epsilon y)\right) v w_{\epsilon} d y\right| & \leq C \epsilon\|v\|_{L^{2}(\tilde{\Omega} \backslash \bar{B})}\left\|\nabla_{y} w_{\epsilon}\right\|_{L^{2}(\tilde{\Omega})} \\
& \leq C \epsilon^{1 / 2}\left\|\nabla_{y} w_{\epsilon}\right\|_{L^{2}(\tilde{\Omega})}
\end{aligned}
$$

The second term is bounded by

$$
\begin{aligned}
\epsilon\left|\int_{\tilde{\Omega} \backslash \bar{B}}\left(\nabla_{x} \gamma(\epsilon y)\right) v \cdot \nabla_{y} w_{\epsilon} d y\right| & \leq C \epsilon\|v\|_{L^{2}(\tilde{\Omega} \backslash \bar{B})}|| \nabla_{y} w_{\epsilon} \|_{L^{2}(\tilde{\Omega} \backslash \bar{B})} \\
& \leq C \epsilon^{1 / 2}\left\|\nabla_{y} w_{\epsilon}\right\|_{L^{2}(\tilde{\Omega})} .
\end{aligned}
$$

To obtain a bound on the third integral, we change variables back to the original domain. This yields

$$
\begin{aligned}
\epsilon\left|\int_{\partial \tilde{\Omega}}\left(\nabla_{x} \gamma(\epsilon y) \cdot \nu_{y}\right) v w_{\epsilon} d \sigma_{y}\right| & =\epsilon^{2-n}\left|\int_{\partial \Omega}\left(\nabla_{x} \gamma(x) \cdot \nu_{x}\right) v(x / \epsilon) w_{\epsilon}(x / \epsilon) d \sigma_{x}\right| \\
& \leq C \epsilon^{2-n}|| v(x / \epsilon)\left\|_{L^{2}(\partial \Omega)}\right\| w_{\epsilon}(x / \epsilon) \|_{L^{2}(\partial \Omega)} \\
& \leq C \epsilon^{2-n}\|v(x / \epsilon)\|_{L^{2}(\partial \Omega)}\left\|\nabla_{x} w_{\epsilon}(x / \epsilon)\right\|_{L^{2}(\Omega)}
\end{aligned}
$$

For the last inequality we have used the fact that $\int_{\partial \Omega} w_{\epsilon}(x / \epsilon) d \sigma_{x}=0$. We have

$$
\left\|\nabla_{x} w_{\epsilon}(x / \epsilon)\right\|_{L^{2}(\Omega)}=\epsilon^{n / 2-1}\left\|\nabla_{y} w_{\epsilon}(y)\right\|_{L^{2}(\tilde{\Omega})},
$$

and due to the decay of $v$ we also have

$$
\begin{gathered}
\|v(x / \epsilon)\|_{L^{\infty}(\partial \Omega)} \leq C \epsilon \quad n=2, \\
\|v(x / \epsilon)\|_{L^{\infty}(\partial \Omega)} \leq C \epsilon^{n-2} \quad n \geq 3 .
\end{gathered}
$$

These estimates, along with (38) and (39), give

$$
\epsilon\left|\int_{\partial \tilde{\Omega}}\left(\nabla_{x} \gamma(\epsilon y) \cdot \nu_{y}\right) v w_{\epsilon} d \sigma_{y}\right| \leq C \epsilon^{1 / 2}|| \nabla_{y} w_{\epsilon} \|_{L^{2}(\tilde{\Omega})}
$$

for any $n \geq 2$. For the fourth term, we obtain the estimate

$$
\begin{aligned}
\left|\int_{\partial B} \epsilon\left(\nabla_{x} \gamma(\epsilon y) \cdot \nu_{y}\right) v w_{\epsilon} d \sigma_{y}\right| & \leq C \epsilon\left\|w_{\epsilon}\right\|_{L^{2}(\partial B)} \\
& \leq C \epsilon\left\|w_{\epsilon}\right\|_{H^{1}(B)} .
\end{aligned}
$$

To estimate the fifth term, we again change variables and obtain

$$
\begin{aligned}
\left|\int_{\partial \tilde{\Omega}} \gamma(\epsilon y) \frac{\partial v}{\partial \nu_{y}} w_{\epsilon} d \sigma_{y}\right| & =\epsilon^{1-n}\left|\int_{\partial \Omega} \gamma(x) \frac{\partial v}{\partial \nu_{y}} w_{\epsilon}(x / \epsilon) d \sigma_{x}\right| \\
& \leq C \epsilon^{1-n}\left\|\frac{\partial v}{\partial \nu_{y}}(x / \epsilon)\right\|_{L^{2}(\partial \Omega)}\left\|w_{\epsilon}(x / \epsilon)\right\|_{L^{2}(\partial \Omega)} \\
& \leq C \epsilon^{1-n}\left\|\frac{\partial v}{\partial \nu_{y}}(x / \epsilon)\right\|_{L^{2}(\partial \Omega)}\left\|\nabla_{x} w_{\epsilon}(x / \epsilon)\right\|_{L^{2}(\Omega)}
\end{aligned}
$$


Now note that from (15) it follows that

$$
\begin{gathered}
\left\|\frac{\partial v}{\partial \nu_{y}}(x / \epsilon)\right\|_{L^{\infty}(\partial \Omega)} \leq C \epsilon^{2} \quad n=2, \\
\left\|\frac{\partial v}{\partial \nu_{y}}(x / \epsilon)\right\|_{L^{\infty}(\partial \Omega)} \leq C \epsilon^{n-1} \quad n \geq 3 .
\end{gathered}
$$

In combination with (39) and (42) these estimates imply

$$
\left|\int_{\partial \tilde{\Omega}} \gamma(\epsilon y) \frac{\partial v}{\partial \nu_{y}} w_{\epsilon} d \sigma_{y}\right| \leq C \epsilon^{1 / 2}\left\|\nabla_{y} w_{\epsilon}\right\|_{L^{2}(\tilde{\Omega})},
$$

for any $n \geq 2$. Finally, applying Taylor's theorem to estimate the sixth integral, we obtain

$$
\begin{aligned}
\left|\int_{\partial B}(\gamma(\epsilon y)-\gamma(0)) \frac{\partial v^{+}}{\partial \nu_{y}} w_{\epsilon} d \sigma_{y}\right| & =\epsilon\left|\int_{\partial B} y \cdot \nabla_{x} \gamma\left(\zeta_{x}\right) \frac{\partial v}{\partial \nu_{y}} w_{\epsilon} d \sigma_{y}\right| \\
& \leq C \epsilon\left\|w_{\epsilon}\right\|_{H^{1}(B)}
\end{aligned}
$$

where we have used the fact that (by elliptic regularity) $\frac{\partial v^{+}}{\partial \nu_{y}}$ is in $L^{2}(\partial B)$. A Combination of the identity (35) with the estimates (36)- (37), (40)-(41) and (43) $-(44)$ yields

$$
\left|\int_{\tilde{\Omega}} \hat{\gamma}_{\epsilon} \nabla_{y} w_{\epsilon} \cdot \nabla_{y} w_{\epsilon} d y\right| \leq C \epsilon^{1 / 2}|| \nabla_{y} w_{\epsilon}\left\|_{L^{2}(\tilde{\Omega})}+C \epsilon\right\| w_{\epsilon} \|_{H^{1}(B)} .
$$

From the previous lemma and that fact that the constants $c_{\epsilon}$ are uniformly bounded it follows that $w_{\epsilon}$ is bounded in $H^{1}(B), 0<\epsilon<\epsilon_{0}$. Based on the above estimate we now conclude that

$$
\left\|\nabla_{y} w_{\epsilon}\right\|_{L^{2}(\tilde{\Omega})} \leq C \epsilon^{1 / 2} \quad 0<\epsilon<\epsilon_{0}
$$

which implies the theorem.

\section{An asymptotic formula for the voltage potential}

We are now ready to derive an asymptotic formula for $u_{\epsilon}(z)$ for those points, $z$, a fixed distance, $d$, away from the inhomogeneities. We shall initially consider the case in which $u_{\epsilon}$ is the potential corresponding to a conductor having a single inhomogeneity, $\epsilon B$, that is "centered" at the origin.

Let $N(\cdot, z)$ denote the Neumann function for $\Omega$ corresponding to a Dirac mass at the point $z$ and cofficient $\gamma$. That is, $N(x, z)$ is the solution to

$$
\begin{aligned}
-\nabla_{x} \cdot \gamma(x) \nabla_{x} N(x, z) & =\delta_{z} \text { in } \Omega, \\
\gamma(x) \frac{\partial N(x, z)}{\partial \nu_{x}} & =-\frac{1}{|\partial \Omega|} \text { on } \partial \Omega .
\end{aligned}
$$


We normalize $N(x, z)$ by requiring that $\int_{\partial \Omega} N(x, z) d \sigma_{x}=0$. Whenever no confusion is possible we shall use the simpler notation $10 N(x)=N(x, z)$. Integrating by parts and using the fact that $\int_{\partial \Omega} u_{\epsilon} d \sigma_{x}=0$, we get

$$
\begin{aligned}
u_{\epsilon}(z) & =-\int_{\Omega} u_{\epsilon} \nabla_{x} \cdot\left(\gamma \nabla_{x} N\right) d x \\
& =\int_{\Omega} \gamma \nabla_{x} u_{\epsilon} \cdot \nabla_{x} N d x-\int_{\partial \Omega} u_{\epsilon} \gamma \frac{\partial N}{\partial \nu_{x}} d \sigma_{x} \\
& =\int_{\Omega \backslash \epsilon B} \gamma \nabla_{x} u_{\epsilon} \cdot \nabla_{x} N d x+\int_{\epsilon \bar{B}} \gamma \nabla_{x} u_{\epsilon} \cdot \nabla_{x} N d x
\end{aligned}
$$

We note that the third identity above is the only point in the derivation of the asymptotic formula for $u_{\epsilon}$ where we use the specific form of the boundary condition for $N$. If one is content with the presence of the term $-\int_{\partial \Omega} u_{\epsilon} \gamma \frac{\partial N}{\partial \nu_{x}} d \sigma_{x}$ then it suffices that $N(x, z)$ be a solution to $-\nabla_{x} \cdot \gamma(x) \nabla_{x} N(x, z)=\delta_{z}$ which is smooth in $\bar{\Omega} \backslash\{z\}$. We shall make use of this observation later. Repeated integration by parts in (45) yields

$$
\begin{aligned}
u_{\epsilon}(z)= & \int_{\partial \Omega} \psi N d \sigma_{x}-\int_{\partial(\epsilon B)} \gamma \frac{\partial u_{\epsilon}}{\partial \nu_{x}} N d \sigma_{x}+\int_{\epsilon B} \gamma \nabla_{x} u_{\epsilon} \cdot \nabla_{x} N d x \\
= & \int_{\partial \Omega} \psi N d \sigma_{x}-\int_{\partial(\epsilon B)} \gamma \frac{\partial u_{\epsilon}}{\partial \nu_{x}} N d \sigma_{x} \\
& \quad+\int_{\epsilon B}(\gamma(x)-\gamma(0)) \nabla_{x} u_{\epsilon} \cdot \nabla_{x} N d x+\int_{\epsilon B} \gamma(0) \nabla_{x} u_{\epsilon} \cdot \nabla_{x} N d x \\
= & \int_{\partial \Omega} \psi N d \sigma_{x}-\int_{\partial(\epsilon B)} \gamma \frac{\partial u_{\epsilon}}{\partial \nu_{x}} N d \sigma_{x} \\
& \quad+\int_{\epsilon B}(\gamma(x)-\gamma(0)) \nabla_{x} u_{\epsilon} \cdot \nabla_{x} N d x+\int_{\partial(\epsilon B)} \gamma(0) \frac{\partial u_{\epsilon}{ }^{-}}{\partial \nu_{x}} N d \sigma_{x}
\end{aligned}
$$

where $\nu$ denotes the outward unit normal to both $\Omega$ and $\epsilon B$. If we expand $\gamma(x)=\gamma(0)+x \cdot \nabla_{x} \gamma\left(\zeta_{x}\right), \zeta_{x} \in \epsilon B$, and make the change of variable $x=\epsilon y$ then the second to last term in (46) may be estimated by

$$
\begin{aligned}
\left|\int_{\epsilon B}(\gamma(x)-\gamma(0)) \nabla_{x} u_{\epsilon} \cdot \nabla_{x} N d x\right| & =\epsilon^{n+1}\left|\int_{B}\left(y \cdot \nabla_{x} \gamma\left(\zeta_{x}\right)\right) \nabla_{x} u_{\epsilon}(\epsilon y) \cdot \nabla_{x} N(\epsilon y) d y\right| \\
& \leq C \epsilon^{n+1}\left\|\nabla_{x} u_{\epsilon}(\epsilon y)\right\|_{L^{2}(B)}\left\|\nabla_{x} N(\epsilon y)\right\|_{L^{2}(B)} .
\end{aligned}
$$

From Lemma 1 we have

$$
\int_{\epsilon B}\left|\nabla_{x}\left(U-u_{\epsilon}\right)\right|^{2} d x \leq C \epsilon^{n}
$$

Changing variables we obtain

$$
\int_{B}\left|\nabla_{x}\left(U-u_{\epsilon}\right)(\epsilon y)\right|^{2} d y \leq C
$$


and therefore,

$$
\int_{B}\left|\nabla_{x} u_{\epsilon}(\epsilon y)\right|^{2} d x \leq C
$$

Since $z$ is bounded away from $\epsilon B$, in the sense that $\operatorname{dist}(z, \epsilon B) \geq d>0$, it now follows that

$$
\left|\int_{\epsilon B}(\gamma(x)-\gamma(0)) \nabla_{x} u_{\epsilon} \nabla_{x} N(x, z) d x\right| \leq C \epsilon^{n+1}|| \nabla_{x} N(\epsilon y, z) \|_{L^{2}(B)} \leq C \epsilon^{n+1} .
$$

Substituting this into (46) and using the boundary condition $\gamma(x) \frac{\partial u_{\epsilon}^{+}}{\partial \nu_{x}}=k \frac{\partial u_{\epsilon}^{-}}{\partial \nu_{x}}$ on $\partial(\epsilon B)$, we obtain

$$
\begin{aligned}
u_{\epsilon}(z) & =\int_{\partial \Omega} \psi N d \sigma_{x}-\int_{\partial(\epsilon B)} \gamma{\frac{\partial u_{\epsilon}}{\partial \nu_{x}}}^{+} N d \sigma_{x}+\int_{\partial(\epsilon B)} \gamma(0) \frac{\partial u_{\epsilon}^{-}}{\partial \nu_{x}} N d \sigma_{x}+O\left(\epsilon^{n+1}\right) \\
& =\int_{\partial \Omega} \psi N d \sigma_{x}+\int_{\partial(\epsilon B)}(\gamma(0)-k) \frac{\partial u_{\epsilon}{ }^{-}}{\partial \nu_{x}} N d \sigma_{x}+O\left(\epsilon^{n+1}\right) .
\end{aligned}
$$

Now let $r_{\epsilon}(x)=u_{\epsilon}(x)-U(x)-\epsilon v\left(\frac{x}{\epsilon}\right)$, where $v$ is the function defined by (13). Then

$$
\begin{aligned}
u_{\epsilon}(z)=\int_{\partial \Omega} \psi N d \sigma_{x} & +\int_{\partial(\epsilon B)}(\gamma(0)-k)\left(\frac{\partial U}{\partial \nu_{x}}+{\frac{\partial v^{-}}{\partial \nu_{y}}}^{-}(x / \epsilon)\right) N d \sigma_{x} \\
& +\int_{\partial(\epsilon B)}(\gamma(0)-k) \frac{\partial r_{\epsilon}}{\partial \nu_{x}} N d \sigma_{x}+O\left(\epsilon^{n+1}\right)
\end{aligned}
$$

Integrating the last term in (47) by parts, we obtain

$$
\begin{aligned}
\int_{\partial(\epsilon B)}(\gamma(0)-k) \frac{\partial r_{\epsilon}{ }^{-}}{\partial \nu_{x}} N d \sigma_{x}= & \int_{\epsilon B}(\gamma(0)-k) \nabla_{x} r_{\epsilon} \nabla_{x} N d x \\
& +\int_{\epsilon B}(\gamma(0)-k) \triangle_{x} r_{\epsilon} N d x
\end{aligned}
$$

Changing variables and using the fact that $\triangle_{x} r_{\epsilon}=-\triangle_{x} U$ in $\epsilon B$ we have

$$
\begin{aligned}
\int_{\partial(\epsilon B)}(\gamma(0)-k) \frac{\partial r_{\epsilon}}{\partial \nu_{x}} N d \sigma_{x}= & \epsilon^{n-1} \int_{B}(\gamma(0)-k) \nabla_{y} r_{\epsilon}(\epsilon y) \nabla_{x} N(\epsilon y) d y \\
& -\epsilon^{n} \int_{B}(\gamma(0)-k) \triangle_{x} U(\epsilon y) N(\epsilon y) d y \\
= & -\epsilon^{n} \int_{B}(\gamma(0)-k) \triangle_{x} U(\epsilon y) N(\epsilon y) d y+O\left(\epsilon^{n+\frac{1}{2}}\right)
\end{aligned}
$$

In the last inequality we have used the estimate $\left\|\nabla_{y} r_{\epsilon}(\epsilon y)\right\|_{L^{2}(B)} \leq C \epsilon^{3 / 2}$ (which follows immediately from Theorem 1) as well as the fact that $\nabla_{x} N(\epsilon y)=\nabla_{x} N(\epsilon y, z)$ is uniformly bounded on $B$ (since $z$ is bounded away from $\epsilon B$ ). Expanding the 
Neumann function, $N$, in a Taylor series about the origin, we see that the last term in equation (47) can be written

$$
\int_{\partial(\epsilon B)}(\gamma(0)-k) \frac{\partial r_{\epsilon}{ }^{-}}{\partial \nu_{x}} N d \sigma_{x}=-\epsilon^{n}(\gamma(0)-k) N(0, z) \int_{B} \triangle_{x} U(\epsilon y) d y+O\left(\epsilon^{n+\frac{1}{2}}\right)
$$

and that the next to last term in (47) can be written

$$
\begin{aligned}
\int_{\partial(\epsilon B)}(\gamma(0)-k) & \left(\frac{\partial U}{\partial \nu_{x}}+{\frac{\partial v^{-}}{\partial \nu_{y}}}^{-}(x / \epsilon)\right) N d \sigma_{x} \\
= & \epsilon^{n-1} \int_{\partial B}(\gamma(0)-k)\left(\frac{\partial U}{\partial \nu_{x}}(\epsilon y)+{\left.\frac{\partial v^{-}}{\partial \nu_{y}}\right) N(\epsilon y, z) d \sigma_{y}}_{=} \epsilon^{n-1}(\gamma(0)-k) N(0, z) \int_{\partial B}\left(\frac{\partial U}{\partial \nu_{x}}(\epsilon y)+{\frac{\partial v^{-}}{\partial \nu_{y}}}^{-}\right) d \sigma_{y}\right. \\
& +\epsilon^{n}(\gamma(0)-k) \nabla_{x} N(0, z) \cdot \int_{\partial B}\left(\frac{\partial U}{\partial \nu_{x}}(\epsilon y)+{\frac{\partial v^{-}}{\partial \nu_{y}}}^{-}\right) y d \sigma_{y}+O\left(\epsilon^{n+1}\right) .
\end{aligned}
$$

Now noting that $\int_{\partial B} \frac{\partial U}{\partial \nu_{x}}(\epsilon y) d \sigma_{y}=\epsilon \int_{B} \triangle_{x} U(\epsilon y) d y$ and $\int_{\partial B} \frac{\partial v^{-}}{\partial \nu_{y}} d \sigma_{y}=0$, we have, upon substitution into (49)

$$
\begin{aligned}
\int_{\partial(\epsilon B)}(\gamma(0)-k)( & \left.\frac{\partial U}{\partial \nu_{x}}+{\frac{\partial v}{\partial \nu_{y}}}^{-}(x / \epsilon)\right) N d \sigma_{x}=\epsilon^{n}(\gamma(0)-k) N(0, z) \int_{B} \triangle_{x} U(\epsilon y) d y \\
& +\epsilon^{n}(\gamma(0)-k) \nabla_{x} N(0, z) \cdot \int_{\partial B}\left(\frac{\partial U}{\partial \nu_{x}}(\epsilon y)+{\frac{\partial v^{-}}{\partial \nu_{y}}}^{-}\right) y d \sigma_{y}+O\left(\epsilon^{n+1}\right) .
\end{aligned}
$$

Inserting (48) and (50) into (47) and envoking the jump condition for the derivative of $v$, given in (13), we get

$$
\begin{aligned}
u_{\epsilon}(z)= & \int_{\partial \Omega} \psi N d \sigma_{x}+\epsilon^{n}(\gamma(0)-k) \nabla_{x} N(0, z) \cdot \int_{\partial B}\left(\frac{\partial U}{\partial \nu_{x}}(\epsilon y)+\frac{\partial v}{\partial \nu_{y}}\right) y d \sigma_{y}+O\left(\epsilon^{n+\frac{1}{2}}\right) \\
= & \int_{\partial \Omega} \psi N d \sigma_{x}+\epsilon^{n}(\gamma(0)-k) \nabla_{x} N(0, z) \cdot\left[\int_{\partial B} \nabla_{x} U(0) \cdot \nu_{y} y d \sigma_{y}\right. \\
& \left.\quad+\int_{\partial B} \frac{1}{k}\left((\gamma(0)-k) \nabla_{x} U(0) \cdot \nu_{y}+\gamma(0) \frac{\partial v^{+}}{\partial \nu_{y}}\right) y d \sigma_{y}\right]+O\left(\epsilon^{n+\frac{1}{2}}\right) \\
= & \int_{\partial \Omega} \psi N d \sigma_{x}+\epsilon^{n} \gamma(0) \frac{(\gamma(0)-k)}{k} \nabla_{x} N(0, z) \cdot \int_{\partial B}\left(\nabla_{x} U(0) \cdot \nu_{y}+{\frac{\partial v}{\partial \nu_{y}}}^{+}\right) y d \sigma_{y} \\
& +O\left(\epsilon^{n+\frac{1}{2}}\right) .
\end{aligned}
$$

Let $\phi_{j}$ denote the solution to

$$
\begin{gathered}
\Delta_{y} \phi_{j}=0 \quad \text { in } B, \quad \Delta_{y} \phi_{j}=0 \quad \text { in } \quad \Re^{n} \backslash \bar{B} \\
\phi_{j} \text { is continuous across } \partial B
\end{gathered}
$$




$$
\begin{aligned}
& \frac{\gamma(0)}{k}{\frac{\partial \phi_{j}}{\partial \nu_{y}}}^{+}-\frac{\partial{\phi_{j}}^{-}}{\partial \nu_{y}}=-\nu_{j} \text { on } \partial B \\
& \lim _{|y| \rightarrow \infty} \phi_{j}(y)=0 .
\end{aligned}
$$

The existence and uniqueness of $\phi_{j}$ is established in a manner completely similar to that of $v$ (cf. [5] or [11]). We note that $\phi_{j}$ and its first derivatives decay at $\infty$ in the same way as $v$ and its first derivatives do (cf. (14) and (15)). It follows immediately from the definitions of $v$ and $\phi_{j}$ that

$$
v(y)=\frac{\gamma(0)-k}{k} \Sigma_{j=1}^{n} \frac{\partial U}{\partial x_{j}}(0) \phi_{j}(y)
$$

and so (51) may be rewritten

$$
\begin{aligned}
u_{\epsilon}(z)=\epsilon^{n} \gamma(0) \frac{\gamma(0)-k}{k} & \nabla_{x} N(0, z) \cdot M \nabla_{x} U(0) \\
& +\int_{\partial \Omega} \psi(x) N(x, z) d \sigma_{x}+O\left(\epsilon^{n+\frac{1}{2}}\right),
\end{aligned}
$$

where the (rescaled) polarization tensor $M=m_{i j}$ is given by

$$
\begin{aligned}
m_{i j} & =\int_{\partial B}\left(y_{i} \nu_{j}+\frac{\gamma(0)-k}{k} y_{i}{\frac{\partial \phi_{j}}{\partial \nu}}^{+}\right) d \sigma_{y} \\
& =|B| \delta_{i j}+\left(\frac{\gamma(0)}{k}-1\right) \int_{\partial B} y_{i}{\frac{\partial \phi_{j}}{\partial \nu}}^{+} d \sigma_{y} .
\end{aligned}
$$

The term $O\left(\epsilon^{n+\frac{1}{2}}\right)$ is bounded by $C_{d} \epsilon^{n+\frac{1}{2}}$ uniformly in $z \in \Omega \cap\{\operatorname{dist}(z, \epsilon B) \geq d>$ $0\}$, and so is its derivatives (the latter fact easily follows from the first by elliptic apriori estimates). By continuity the formula (53) also holds for $z \in \partial \Omega$ (provided $\operatorname{dist}(\partial \Omega, \epsilon B) \geq d>0)$. We note that the (rescaled) polarization tensor, $M$, only depends on the ratio $r=\gamma(0) / k$, not on the individual conductivities $\gamma(0)$ and $k$. We shall occasionally use the notation $M(r)$ to make this dependence on $r=\gamma(0) / k$ more explicit. If the single inhomogeneity is given by $z_{1}+\epsilon B$ (instead of $B$ ) then the only effect is to change 0 to $z_{1}$. It is not essential that $B$ be strictly star-shaped with respect to the origin, it suffices that $B$ be strictly star-shaped with respect to any point. We simply write $B=p+\tilde{B}$, where $\tilde{B}=B-p$ is now star-shaped with respect to the origin. The inhomogeneity $z_{1}+\epsilon B$ may now be written as $z_{1}+\epsilon p+\epsilon \tilde{B}$; the order $\epsilon$ translation does not materially affect the previous argument and $\tilde{B}$ has the same polarization tensor as $B$, so the formula (53) stays unchanged. In case of more than one inhomogeneity, say $\omega_{\epsilon}=\cup_{i=1}^{m}\left(z_{i}+\epsilon B_{i}\right)$ (with $z_{i}+\epsilon B_{i}$ of conductivity $k_{i}$ ) the previous argument may very simply be changed to proceed inductively one inhomogeneity at a time. In summary we have therefore proven

Theorem 2. Suppose $n=2$ or 3 and suppose the domains $B_{i}, i=1, \ldots, m$, are strictly star-shaped. Also suppose the points $z_{i} \in \Omega, i=1 \ldots m$, are mutually 
distinct and satisfy (4). Then for any $z \in \bar{\Omega} \backslash\left\{z_{i}\right\}_{i=1}^{m}$

$$
\begin{aligned}
u_{\epsilon}(z)=\epsilon^{n} \Sigma_{i=1}^{m} & \gamma\left(z_{i}\right) \frac{\gamma\left(z_{i}\right)-k_{i}}{k_{i}} \nabla_{x} N\left(z_{i}, z\right) \cdot M_{i} \nabla_{x} U\left(z_{i}\right) \\
& +\int_{\partial \Omega} \psi(x) N(x, z) d \sigma_{x}+O\left(\epsilon^{n+\frac{1}{2}}\right)
\end{aligned}
$$

for $\epsilon$ sufficiently small. The term $O\left(\epsilon^{n+\frac{1}{2}}\right)$ and its derivatives are uniformly bounded by $C_{d} \epsilon^{n+\frac{1}{2}}$ on $\bar{\Omega} \cap\left\{\operatorname{dist}\left(z, \omega_{\epsilon}\right) \geq d>0\right\}$. The rescaled polarization tensor, $M_{i}$, corresponding to the $i^{\text {th }}$ inhomogeneity is calculated just as before, only with $B$ replaced by $B_{i}$ and $k$ by $k_{i}$.

\section{Remark 1}

An interesting special case is when the background conductivity, $\gamma$, is constant, the inhomogeneities have a common conductivity $k$, and all the domains $B_{i}$ have been obtained from the same B by a dilatation and a rotation. Let $\rho_{i}>0$ be the dilatation parameter and $Q_{i}$ the rotation corresponding to the $\mathrm{i}$-th inhomogeneity, i.e., $B_{i}=\rho_{i} Q_{i} B$. In this case it is not difficult to compute that $M_{i}=\rho_{i}^{n} Q_{i} M Q_{i}^{T}$, where $M$ is the (rescaled) polarization tensor correponding to $B$ and conductivity ratio $r=\gamma / k$. Thus the representation formula for $u_{\epsilon}$ becomes

$$
\begin{aligned}
u_{\epsilon}(z)=\Sigma_{i=1}^{m}\left(\epsilon \rho_{i}\right)^{n} \gamma \frac{\gamma-k}{k} & \nabla_{x} N\left(z_{i}, z\right) \cdot Q_{i} M Q_{i}^{T} \nabla_{x} U\left(z_{i}\right) \\
& +\int_{\partial \Omega} \psi(x) N(x, z) d \sigma_{x}+O\left(\epsilon^{n+\frac{1}{2}}\right) .
\end{aligned}
$$

\section{Remark 2}

As pointed out earlier representation formuli similar to (54) may be obtained using other fundamental solutions for the operator $-\nabla \cdot \gamma \nabla$ than the Neumann function, $N$. The only difference will be the presence of a second boundary integral. A particularly simple case is when the background conductivity $\gamma$ is constant. In that situation it is natural in place of $N(x, y)$ to use

$$
\Phi(x, y)= \begin{cases}-\frac{1}{2 \pi \gamma} \log |x-y|, & n=2 \\ \frac{1}{4 \pi \gamma}|x-y|^{-1}, & n=3\end{cases}
$$

The representation formula for $u_{\epsilon}$ now becomes

$$
\begin{aligned}
u_{\epsilon}(z)=\epsilon^{n} \sum_{i=1}^{m} \gamma \frac{\gamma-k_{i}}{k_{i}} \nabla_{x} \Phi\left(z_{i}, z\right) \cdot M_{i} \nabla_{x} U\left(z_{i}\right) & \\
& \quad+\int_{\partial \Omega} \psi(x) \Phi(x, z) d \sigma_{x}-\int_{\partial \Omega} u_{\epsilon}(x) \gamma \frac{\partial \Phi}{\partial \nu_{x}}(x, z) d \sigma_{x}+O\left(\epsilon^{n+\frac{1}{2}}\right)
\end{aligned}
$$


or by subtraction of $U(z)$ on both sides

$$
\begin{aligned}
u_{\epsilon}(z)-U(z)=\epsilon^{n} \sum_{i=1}^{m} & \gamma \frac{\gamma-k_{i}}{k_{i}} \nabla_{x} \Phi\left(z_{i}, z\right) \cdot M_{i} \nabla_{x} U\left(z_{i}\right) \\
& -\int_{\partial \Omega}\left(u_{\epsilon}(x)-U(x)\right) \gamma \frac{\partial \Phi}{\partial \nu_{x}}(x, z) d \sigma_{x}+O\left(\epsilon^{n+\frac{1}{2}}\right) .
\end{aligned}
$$

The rearranged formula

$$
\begin{aligned}
u_{\epsilon}(z) & -U(z)+\int_{\partial \Omega}\left(u_{\epsilon}(x)-U(x)\right) \gamma \frac{\partial \Phi}{\partial \nu_{x}}(x, z) d \sigma_{x} \\
= & \epsilon^{n} \sum_{i=1}^{m} \gamma \frac{\gamma-k_{i}}{k_{i}} \nabla_{x} \Phi\left(z_{i}, z\right) \cdot M_{i} \nabla_{x} U\left(z_{i}\right)+O\left(\epsilon^{n+\frac{1}{2}}\right),
\end{aligned}
$$

may be thought of as a representation formula for $L\left(u_{\epsilon}-U\right)$ where $L(V)$ is defined by $L(V)=V+\int_{\partial \Omega} V \gamma \frac{\partial \Phi}{\partial \nu_{x}} d \sigma_{x}$. For the inverse problem, when $u_{\epsilon}-U$ and thus $L\left(u_{\epsilon}-U\right)$ is known on the entire boundary, then the formula (55) becomes a very effective tool for the identification of the $z_{i}$ and properties of $\frac{\gamma-k_{i}}{k_{i}} M_{i}$ (as we shall see in the last section of this paper). It should be pointed out that the formula (55) is not well suited for the inverse problem when the data $u_{\epsilon}-U$ is only known on part of $\partial \Omega$, nor is it suited to prove the kind of continuous dependence results discussed in section 7 . For those purposes the version of the representation formula stated in Theorem 2 is much more useful.

\section{Properties of the polarization tensor}

In this section we analyse in some detail the properties of the $n \times n$ matrix $M$, introduced in the previous section. We have already referred to this matrix as the rescaled polarization tensor associated to $B$ and conductivity ratio $r=\gamma(0) / k$. The polarization tensor itself is defined to be $(r-1) M$. We recall that $M=\left\{m_{i j}\right\}$ is given by

$$
m_{i j}=|B| \delta_{i j}+(r-1) \int_{\partial B} y_{i}{\frac{\partial \phi_{j}}{\partial \nu}}^{+} d \sigma_{y} .
$$

We begin by showing

\section{Lemma 5 . The polarization tensor is symmetric.}

Proof. In order to verify the symmetry, we shall rewrite the integral appearing in the definition of $M$. Using the jump condition for $\frac{\partial \phi_{j}}{\partial \nu}$ on $\partial B$ and integrating by parts we obtain

$$
\begin{aligned}
\int_{\partial B} y_{i}{\frac{\partial \phi_{j}}{\partial \nu}}^{+} d \sigma_{y} & =\frac{1}{r} \int_{\partial B} y_{i}\left(\frac{\partial \phi_{j}^{-}}{\partial \nu}-\nu_{j}\right) d \sigma_{y} \\
& =\frac{1}{r}\left(\int_{\partial B} \phi_{j} \nu_{i} d \sigma_{y}-\int_{\partial B} y_{i} \nu_{j} d \sigma_{y}\right) \\
& =\frac{1}{r}\left(\int_{\partial B} \phi_{j} \nu_{i} d \sigma_{y}-|B| \delta_{i j}\right)
\end{aligned}
$$


Substitution of (57) into (56) and simplification yields

$$
m_{i j}=\frac{1}{r}|B| \delta_{i j}+\left(1-\frac{1}{r}\right) \int_{\partial B} \phi_{j} \nu_{i} d \sigma .
$$

To establish the symmetry of $M$ (or $(r-1) M$ ) it now suffices to show that $\int_{\partial B} \phi_{j} \nu_{i} d \sigma=\int_{\partial B} \phi_{i} \nu_{j} d \sigma, \quad 1 \leq i, j \leq n$. Using the jump condition for $\frac{\partial \phi_{j}}{\partial \nu}$ on $\partial B$ once more we get

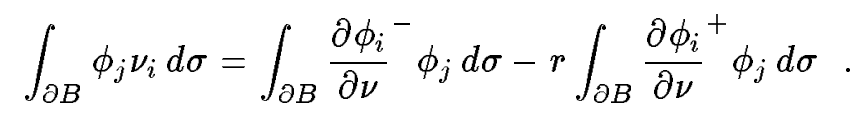

The first integral on the right hand side of (59) may immediately be integrated by parts to become $\int_{\partial B} \phi_{i} \frac{\partial \phi_{j}^{-}}{\partial \nu} d \sigma$. As previously observed $\phi_{j}$ has the following behavior (decay) at infinity

$$
\phi_{j}(y)=\left\{\begin{array}{ll}
O\left(|y|^{-1}\right) & n=2 \\
O\left(|y|^{2-n}\right) & n \geq 3
\end{array} \quad \nabla_{y} \phi_{j}(y)= \begin{cases}O\left(|y|^{-2}\right) & n=2 \\
O\left(|y|^{1-n}\right) & n \geq 3\end{cases}\right.
$$

This decay implies that the second integral on the right hand side of (59) may also be integrated by parts. Altogether we obtain

$$
\begin{aligned}
\int_{\partial B} \phi_{j} \nu_{i} d \sigma & =\int_{\partial B} \frac{\partial \phi_{j}{ }^{-}}{\partial \nu} \phi_{i} d \sigma-r \int_{\partial B} \frac{\partial \phi_{j}{ }^{+}}{\partial \nu} \phi_{i} d \sigma \\
& =\int_{\partial B} \phi_{i} \nu_{j} d \sigma \quad 1 \leq i, j \leq n
\end{aligned}
$$

and therefore, $M$ is symmetric.

We shall use that the polarization tensor is invertible whenever $\gamma(0) \neq k$ $(r \neq 1)$. This follows immediately from

Lemma 6. The rescaled polarization tensor, $M$, is positive definite. Thus, the polarization tensor $(r-1) M$ is positive definite for $r>1(\gamma(0)>k)$ and it is negative definite for $r<1(\gamma(0)<k)$.

Proof. Using (58)and (59) we compute

$$
\sum m_{i j} \zeta_{i} \zeta_{j}=\frac{1}{r}\left(|B||\zeta|^{2}+(r-1)\left[\int_{\partial B} \frac{\partial \chi^{-}}{\partial \nu} \chi d \sigma-r \int_{\partial B} \frac{\partial \chi^{+}}{\partial \nu} \chi d \sigma\right]\right),
$$

where $\chi=\sum \zeta_{i} \phi_{i}$, and $\nu$ is the outer normal to $B$. Integrating by parts we obtain

$$
\sum m_{i j} \zeta_{i} \zeta_{j}=\frac{1}{r}\left(|B||\zeta|^{2}+(r-1)\left[\int_{B}|\nabla \chi|^{2} d y+r \int_{\Re^{n} \backslash \bar{B}}|\nabla \chi|^{2} d y\right]\right) .
$$


It follows directly from this representation that if $r \geq 1(\gamma(0) \geq k)$ then $M$ is positive definite. Note that if $r=1(\gamma(0)=k)$ then $M$ is simply given by

$$
m_{i j}=|B| \delta_{i j}
$$

however, the polarization tensor $(r-1) M$ vanishes.

We now seek a different representation for $M$ that will allow us to verify its positive definiteness in the case $r<1(\gamma(0)<k)$. Rewriting (56) as a single integral and rearranging terms, we have

$$
\begin{aligned}
m_{i j} & =\int_{\partial B}\left(y_{i} \nu_{j}+y_{i}(r-1) \frac{\partial \phi_{j}{ }^{+}}{\partial \nu}\right) d \sigma_{y} \\
& =\int_{\partial B} y_{i}\left((r-1) \nabla \phi_{j}^{+}+\nabla y_{j}\right) \cdot \nu d \sigma_{y}
\end{aligned}
$$

Using the jump condition for $\frac{\partial \phi_{j}}{\partial \nu}$ on $\partial B$ and simplifying we get

$$
\begin{aligned}
m_{i j} & =\int_{\partial B} y_{i}\left(\left(1-\frac{1}{r}\right)\left(\nabla \phi_{j}^{-}-\nabla y_{j}\right)+\nabla y_{j}\right) \cdot \nu d \sigma_{y} \\
& =\frac{1}{r} \int_{\partial B} y_{i}\left((r-1) \nabla \phi_{j}^{-}+\nabla y_{j}\right) \cdot \nu d \sigma_{y}
\end{aligned}
$$

Now note that the above can be rewritten as

$$
\begin{aligned}
m_{i j}=\frac{1}{r}[ & \int_{\partial B}\left((r-1) \phi_{i}+y_{i}\right)\left((r-1) \nabla \phi_{j}^{-}+\nabla y_{j}\right) \cdot \nu d \sigma_{y} \\
& \left.+(1-r) \int_{\partial B} \phi_{i}\left((r-1) \nabla \phi_{j}^{-}+\nabla y_{j}\right) \cdot \nu d \sigma_{y}\right]
\end{aligned}
$$

Application of the divergence theorem to the first integral in (60) yields

$$
\begin{aligned}
& m_{i j}=\frac{1}{r}\left[\int_{B} \nabla\left((r-1) \phi_{i}+y_{i}\right) \cdot \nabla\left((r-1) \phi_{j}+y_{j}\right) d y\right. \\
&\left.\quad+(1-r) \int_{\partial B} \phi_{i}\left((r-1) \nabla \phi_{j}^{-}+\nabla y_{j}\right) \cdot \nu d \sigma_{y}\right] .
\end{aligned}
$$

The second integral on the right hand side may also be further simplied. Using the jump condition for $\frac{\partial \phi_{j}}{\partial \nu}$ on $\partial B$ we obtain

$$
\begin{aligned}
(1-r) \int_{\partial B} \phi_{i}((r & \left.-1) \nabla \phi_{j}^{-}+\nabla y_{j}\right) \cdot \nu d \sigma_{y} \\
& =(1-r) \int_{\partial B} \phi_{i}\left((r-1) \nabla \phi_{j}^{-}+\nabla \phi_{j}^{-}-r \nabla \phi_{j}^{+}\right) \cdot \nu d \sigma_{y} \\
& =(1-r) r \int_{\partial B} \phi_{i}\left(\nabla \phi_{j}^{-}-\nabla \phi_{j}^{+}\right) \cdot \nu d \sigma_{y}
\end{aligned}
$$


Expanding the right hand side of (62) as two separate integrals and integrating by parts (using the decay of $\phi_{j}$ ) we get

$$
\begin{aligned}
(1-r) \int_{\partial B} \phi_{i}((r & \left.-1) \nabla \phi_{j}^{-}+\nabla y_{j}\right) \cdot \nu d \sigma_{y} \\
& =(1-r) r\left(\int_{\partial B} \phi_{i} \frac{\partial \phi_{j}{ }^{-}}{\partial \nu} d \sigma_{y}-\int_{\partial B} \phi_{i}{\frac{\partial \phi_{j}}{\partial \nu}}^{+} d \sigma_{y}\right) \\
& =(1-r) r \int_{\Re^{n}} \nabla \phi_{i} \cdot \nabla \phi_{j} d y .
\end{aligned}
$$

Note that $\nu$ is the outward unit normal to $B$. Substituting back into (61) we finally have

$m_{i j}=\frac{1}{r} \int_{B} \nabla\left((r-1) \phi_{i}+y_{i}\right) \cdot \nabla\left((r-1) \phi_{j}+y_{j}\right) d y+(1-r) \int_{\Re^{n}} \nabla \phi_{i} \cdot \nabla \phi_{j} d y$,

or

$$
\sum m_{i j} \zeta_{i} \zeta_{j}=\frac{1}{r} \int_{B}|\nabla \tilde{\chi}|^{2} d y+(1-r) \int_{\Re^{n}}|\nabla \chi|^{2} d y
$$

where $\tilde{\chi}=\sum \zeta_{i}\left((r-1) \phi_{i}+y_{i}\right)$ and $\chi=\sum \zeta_{i} \phi_{i}$. It follows immediately from (63) that $M$ is also positive definite for $r<1(\gamma(0)<k)$.

\section{Remark 3}

When $B$ is the unit ball we can explicitly determine $M$. In this special case

$$
\phi_{j}=\frac{1}{(n-1) r+1} y_{j} \quad \text { in } B \quad, \quad \phi_{j}=\frac{1}{(n-1) r+1} \frac{y_{j}}{|y|^{n}} \quad \text { in } \Re^{n} \backslash B
$$

and a simple computation then gives

$$
m_{i j}=\frac{\omega_{n}}{(n-1) r+1} \delta_{i j}
$$

where $\omega_{n}$ denotes the area of the unit sphere in $\Re^{n}$. The polarization tensor itself has entries $\delta_{i j} \omega_{n}(r-1) /((n-1) r+1)$.

In [7] we derived an asymptotic expansion for the voltage potential $u_{\epsilon}$ in the special case of inhomogeneities that are either perfectly insulating or perfectly conducting. We shall conclude this section by showing that the approximations we obtained in these cases are entirely the same as those we would obtain by letting $k$ tend towards 0 or $\infty$ in the approximation obtained here. Polarization tensors corresponding to these two extreme cases have been considered many other places in the literature; we refer the reader to [16] and [9]. 


\subsection{The limit as $k \rightarrow 0$.}

Let $\phi_{j}^{(k)} \in C\left(\Re^{n}\right), j=1, \ldots, n$, denote the unique solutions to

$$
\begin{gathered}
\triangle \phi_{j}^{(k)}=0, \text { in } B, \quad \triangle \phi_{j}^{(k)}=0, \text { in } \Re^{n} \backslash \bar{B} \\
\phi_{j}^{(k)} \text { is continuous across } \partial B \\
\frac{\partial \phi_{j}^{(k)}}{\partial \nu}-\frac{k}{\gamma(0)} \frac{\partial \phi_{j}^{(k)}}{\partial \nu}=-\nu_{j}, \text { on } \partial B \\
\lim _{|y| \rightarrow \infty} \phi_{j}^{(k)}(y)=0 .
\end{gathered}
$$

There is a simple relation to the functions $\phi_{j}$ introduced earlier ( $\left.c f .(52)\right)$, namely $\phi_{j}^{(k)}=\frac{\gamma(0)}{k} \phi_{j}$.

Lemma 7. The sequence of functions $\left\{\left.\phi_{j}^{(k)}\right|_{\partial B}\right\}$ converges uniformly, as $k \rightarrow 0$, to $\left.\phi_{j}^{(0)}\right|_{\partial B}$, where $\phi_{j}^{(0)}$ is the solution to the following exterior Neumann problem,

$$
\begin{gathered}
\Delta \phi_{j}^{(0)}=0 \quad \text { in } \Re^{n} \backslash \bar{B}, \\
\frac{\partial \phi_{j}^{(0)}{ }^{+}}{\partial \nu}=-\nu_{j} \quad \text { on } \partial B, \lim _{|y| \rightarrow \infty} \phi_{j}^{(0)}(y)=0 .
\end{gathered}
$$

Proof. We first note that the exterior Neumann problem (65) is uniquely solvable. In $\Re^{2}$, it owes its solvability to the fact that $\int_{\partial B} \nu_{j} d s=0$. Let $X$ be the space of functions

$$
X=\left\{\Psi \in C(\partial B): \int_{\partial B} \Psi d \sigma=0\right\}
$$

Let $\Phi(x, y)$ denote the fundamental solution

$$
\Phi(x, y)= \begin{cases}-\frac{1}{2 \pi} \log |x-y|, & n=2 \\ \frac{1}{(n-2) \omega_{n}}|x-y|^{2-n}, & n \geq 3\end{cases}
$$

$\Phi(x, y)$ is as introduced before, only with $\gamma=1$. Writing $\phi_{j}^{(k)}$ as a single layer potential with density $\Psi_{j}^{(k)} \in X$ we have

$$
\phi_{j}^{(k)}(y)=\int_{\partial B} \Phi(x, y) \Psi_{j}^{(k)}(x) d \sigma_{x}
$$

From classical potential theory $(c f .[5],[11])$ it follows that $\phi_{j}^{(k)}$ is a solution to (64) iff

$$
\begin{aligned}
k \frac{\partial \phi_{j}^{(k)}}{\partial \nu_{y}}-\gamma(0) \frac{\partial \phi_{j}^{(k)}}{\partial \nu_{y}} & =(k-\gamma(0)) \int_{\partial B} \frac{\partial \Phi(x, y)}{\partial \nu_{y}} \Psi_{j}^{(k)}(x) d \sigma_{x}+\frac{k+\gamma(0)}{2} \Psi_{j}^{(k)}(y) \\
& =\gamma(0) \nu_{j}
\end{aligned}
$$


Multiplying equation (66) by $\frac{2}{k+\gamma(0)}$ we obtain

$$
\left(I-A^{(k)}\right) \Psi_{j}^{(k)}=f_{j}^{(k)}
$$

where $A^{(k)}$ is the compact operator $(X \rightarrow X)$ defined by

$$
A^{(k)} \Psi=-2\left(\frac{k-\gamma(0)}{k+\gamma(0)}\right) \int_{\partial B} \frac{\partial \Phi(x, y)}{\partial \nu_{y}} \Psi(x) d \sigma_{x}
$$

and

$$
f_{j}^{(k)}=\frac{2 \gamma(0)}{k+\gamma(0)} \nu_{j}
$$

To see that $A^{(k)}$ maps $X$ into $X$ we use the fact that $\int_{\partial B} \frac{\partial \Phi(x, y)}{\partial \nu_{y}} d \sigma_{y}=-1 / 2$ for $x \in \partial B$. Similarly writing $\phi_{j}^{(0)}(y)=\int_{\partial B} \Phi(x, y) \Psi_{j}^{(0)}(x) d \sigma_{x}$ as a single layer potential with density $\Psi_{j}^{(0)} \in X$ a necessary and sufficient condition that $\phi_{j}^{(0)}$ solves (65) is

$$
\left(I-A^{(0)}\right) \Psi_{j}^{(0)}=f_{j}^{(0)} .
$$

Here $A^{(0)}(X \rightarrow X)$ is the compact operator given by

$$
A^{(0)} \Psi=2 \int_{\partial B} \frac{\partial \Phi(x, y)}{\partial \nu_{y}} \Psi(x) d \sigma_{x}
$$

and

$$
f_{j}^{(0)}=2 \nu_{j}
$$

$j=1, \ldots, n$. To establish the lemma, it now suffices to show that $\Psi_{j}^{(k)}$ converges uniformly to $\Psi_{j}^{(0)}$. From the uniqueness associated with (65) it follows that (67) has at most one solution. By the Fredholm Alternative, $\left(I-A^{(0)}\right)^{-1}$ exists and is bounded. The sequence $A^{(k)}$ converges to $A^{(0)}$ in the operator norm as $k \rightarrow 0$, and consequently, the invertible operators $I-A^{(k)}$ have a uniform bound for their inverses

$$
\left\|\left(I-A^{(k)}\right)^{-1}\right\| \leq C \quad, \quad \forall k \leq 1
$$

A simple estimation now gives

$$
\begin{aligned}
\left\|\Psi_{j}^{(k)}-\Psi_{j}^{(0)}\right\| & \leq\left\|\left(I-A^{(k)}\right)^{-1}\right\|\left\|\left(I-A^{(k)}\right)\left(\Psi_{j}^{(k)}-\Psi_{j}^{(0)}\right)\right\| \\
& \leq C\left(\left\|\left(I-A^{(k)}\right) \Psi_{j}^{(k)}-\left(I-A^{(0)}\right) \Psi_{j}^{(0)}\right\|+\left\|\left(A^{(k)}-A^{(0)}\right) \Psi_{j}^{(0)}\right\|\right) \\
& \leq C\left(\left\|f_{j}^{(k)}-f_{j}^{(0)}\right\|+\left\|\left(A^{(k)}-A^{(0)}\right) \Psi_{j}^{(0)}\right\|\right),
\end{aligned}
$$

where all the norms pertain to the space $X$. The lemma now follows from the facts that $f_{j}^{(k)} \rightarrow f_{j}^{(0)}$ and $A^{(k)} \rightarrow A^{(0)}$ as $k \rightarrow 0$. 
Let $M^{(k)}=\left\{m_{i j}^{(k)}\right\}$ denote the polarization tensor $M^{(k)}=\left(\frac{\gamma(0)}{k}-1\right) M\left(\frac{\gamma(0)}{k}\right)$ (with $M\left(\frac{\gamma(0)}{k}\right)$ being the rescaled polarization tensor introduced earlier). From (58) we get

$$
\begin{aligned}
m_{i j}^{(k)} & =\left(1-\frac{1}{r}\right)|B| \delta_{i j}+\left(1-\frac{1}{r}\right)^{2} r \int_{\partial B} \phi_{j} \nu_{i} d \sigma \\
& =\left(1-\frac{1}{r}\right)|B| \delta_{i j}+\left(1-\frac{1}{r}\right)^{2} \int_{\partial B} \phi_{j}^{(k)} \nu_{i} d \sigma
\end{aligned}
$$

with $r=\frac{\gamma(0)}{k}$. Due to Lemma 7 it follows immediately that the tensor $M^{(k)}$, as $k \rightarrow 0(r \rightarrow \infty)$ converges to the tensor $M^{(0)}=\left\{m_{i j}^{(0)}\right\}$, given by

$$
m_{i j}^{(0)}=|B| \delta_{i j}+\int_{\partial B} \phi_{j}^{(0)} \nu_{i} d \sigma
$$

The latter is exactly the tensor defined in (5.7) of [7]. This means that the present asymptotic formula in the extreme case $k \rightarrow 0$ (and for simplicity, with one inhomogeneity "centered" at the origin)

$$
\begin{aligned}
u_{\epsilon}(z) & \approx \epsilon^{n} \frac{\gamma(0)(\gamma(0)-k)}{k} \nabla_{x} N(0, z) \cdot M\left(\frac{\gamma(0)}{k}\right) \nabla_{x} U(0)+U(z) \\
& =\epsilon^{n} \gamma(0) \nabla_{x} N(0, z) \cdot M^{(k)} \nabla_{x} U(0)+U(z)
\end{aligned}
$$

is entirely consistent with the formula derived in [7] (with $\rho=1$ ). The separate proof of the asymptotic validity of this formula (for " $k=0$ ") is still needed since we have not shown that the term $O\left(\epsilon^{n+\frac{1}{2}}\right)$ is uniform in $k$, as $k \rightarrow 0$.

\subsection{The limit as $k \rightarrow \infty$}

To study the limit of the polarization tensor as $k \rightarrow \infty$ it is convenient to perform a different rescaling of the functions $\phi_{j}$. In this section we let $\phi_{j}^{(k)}$ denote the solution to

$$
\begin{gathered}
\Delta \phi_{j}^{(k)}=0, \quad \text { in } B, \quad \Delta \phi_{j}^{(k)}=0, \quad \text { in } \Re^{n} \backslash \bar{B} \\
\phi_{j}^{(k)} \text { is continuous across } \partial B \\
\frac{\gamma(0)}{k} \frac{\partial \phi_{j}^{(k)^{+}}}{\partial \nu}-\frac{\partial \phi_{j}^{(k)}}{\partial \nu}=-\frac{k-\gamma(0)}{k} \nu_{j}, \quad \text { on } \partial B \\
\lim _{|y| \rightarrow \infty} \phi_{j}^{(k)}(y)=0 .
\end{gathered}
$$

With this new definition $\phi_{j}^{(k)}=\frac{k-\gamma(0)}{k} \phi_{j}$, where $\phi_{j}$ is the solution to (52). Once again, we represent $\phi_{j}^{(k)}$ in the form of a single layer potential

$$
\phi_{j}^{(k)}(y)=\int_{\partial B} \Phi(x, y) \Psi_{j}^{(k)}(x) d \sigma_{x}
$$


$\Psi_{j}^{(k)} \in X$. In this case

$$
\frac{\partial \phi_{j}^{(k)}}{\partial \nu_{y}}=\frac{1}{2} \Psi_{j}^{(k)}(y)+\int_{\partial B} \frac{\partial \Phi(x, y)}{\partial \nu_{y}} \Psi_{j}^{(k)}(x) d \sigma_{x}
$$

and

$$
\frac{\gamma(0)}{k} \frac{\partial \phi_{j}^{(k)}}{\partial \nu_{y}}=-\frac{\gamma(0)}{2 k} \Psi_{j}^{(k)}(y)+\frac{\gamma(0)}{k} \int_{\partial B} \frac{\partial \Phi(x, y)}{\partial \nu_{y}} \Psi_{j}^{(k)}(x) d \sigma_{x}
$$

Insertion of equation (69) and (70) into (68) yields

$$
\frac{k+\gamma(0)}{2 k} \Psi_{j}^{(k)}(y)+\left(1-\frac{\gamma(0)}{k}\right) \int_{\partial B} \frac{\partial \Phi(x, y)}{\partial \nu_{y}} \Psi_{j}^{(k)}(x) d \sigma_{x}=\frac{k-\gamma(0)}{k} \nu_{j}
$$

Multiplication by $2 k /(k+\gamma(0))$ therefore gives the following (necessary and sufficient) equation for $\Psi_{j}$

$$
\Psi_{j}^{(k)}(y)+2 \frac{(k-\gamma(0))}{(k+\gamma(0))} \int_{\partial B} \frac{\partial \Phi(x, y)}{\partial \nu_{y}} \Psi_{j}^{(k)}(x) d \sigma_{x}=2 \frac{k-\gamma(0)}{k+\gamma(0)} \nu_{j}
$$

Letting $k \rightarrow \infty$ we formally obtain

$$
\Psi_{j}^{(\infty)}+A^{(\infty)} \Psi_{j}^{(\infty)}=2 \nu_{j}, \quad \Psi_{j}^{(\infty)} \in X
$$

where

$$
A^{(\infty)} \Psi=2 \int_{\partial B} \frac{\partial \Phi(x, y)}{\partial \nu_{y}} \Psi(x) d \sigma_{x}
$$

Indeed by an argument similar to that in the previous section one can show that $\Psi_{j}^{(k)}$ converges uniformly to $\Psi_{j}^{(\infty)}$, and therefore, $\left.\phi_{j}^{(k)}\right|_{\partial B}$ converges uniformly to $\left.\phi_{j}^{(\infty)}\right|_{\partial B}$, where $\phi_{j}^{(\infty)}$ is the single layer potential with density function $\Psi_{j}^{(\infty)}$. It is also easy to prove that $\frac{\partial \phi_{j}^{(k)}+}{\partial \nu}$ converges uniformly to $\frac{\partial \phi_{j}^{(\infty)}+}{\partial \nu}$ on $\partial B$. From (71) it follows that $\left.\phi_{j}^{(\infty)}\right|_{B}$ solves the interior Neumann problem

$$
\begin{gathered}
\Delta \phi_{j}^{(\infty)}=0 \text { in } B, \\
\frac{\partial \phi_{j}^{(\infty)}-}{\partial \nu}=\nu_{j} \text { on } \partial B .
\end{gathered}
$$

In other words $\phi_{j}^{(\infty)}=y_{j}+c_{j}$ on $\mathrm{B}$, for some constant $c_{j}$. From (71) it follows that $\left.\phi_{j}^{(\infty)}\right|_{\Re^{n} \backslash \bar{B}}$ solves the exterior Neumann problem (for the Laplacian) with boundary data $\frac{\partial \phi_{j}^{(\infty)}+}{\partial \nu}=\nu_{j}-\Psi_{j}^{(\infty)}$, and it satisfies $\phi_{j}^{(\infty)} \rightarrow 0$ as $|y| \rightarrow \infty$ (for $n=2$ this relies on the fact that $\int_{\partial B} \Psi_{j}^{(\infty)} d \sigma=0$ ). Furthermore $\phi_{j}^{(\infty)}$ satisfies the exterior Dirichlet problem (for the Laplacian) with boundary data 
$\left.\phi_{j}^{(\infty)}\right|_{\partial B}=y_{j}+c_{j}$. From this latter observation it follows immediately that $\phi_{j}^{(\infty)}=-\Phi_{j}+c_{j} \phi$ where $\Phi_{j}$ is the solution to the exterior dirichlet problem

$$
\begin{gathered}
\Delta \Phi_{j}=0 \text { in } \Re^{n} \backslash \bar{B}, \\
\Phi_{j}=-y_{j} \text { on } \partial B, \\
\Phi_{j} \quad \text { is harmonic at } \infty,
\end{gathered}
$$

and $\phi$ is the solution to

$$
\begin{gathered}
\Delta \phi=0 \text { in } \Re^{n} \backslash \bar{B}, \\
\phi=1 \text { on } \partial B, \\
\phi \quad \text { is harmonic at } \infty .
\end{gathered}
$$

The requirement that a function, which satisfies the equation $\triangle u=0$ outside $\bar{B}$, be harmonic at $\infty$ is equivalent to the the requirement that its Kelvin Transform have a removable singularity at 0 . For $n=2$ the function $\phi$ is identically equal to the constant 1 , and so

$$
\phi_{j}^{(\infty)}=-\Phi_{j}+c_{j}
$$

The fact that $\lim _{|y| \rightarrow \infty} \phi_{j}^{(\infty)}(y)=0$, uniquely determines $c_{j}$. For $n \geq 3$ the requirement that $\phi$ be harmonic at $\infty$ is equivalent to the requirement that $\lim _{|y| \rightarrow \infty} \phi(y)=0$, and so the function $\phi$ is nontrivial.

As noted before we have $\frac{\partial \phi_{j}^{(\infty)}+}{\partial \nu}=\nu_{j}-\Psi_{j}^{(\infty)}$ on $\partial B, ;$ since $\Psi_{j}^{(\infty)} \in X$ it now follows that $\int_{\partial B} \frac{\partial \phi_{j}^{(\infty)}+}{\partial \nu} d \sigma=0$. Therefore

$$
0=\int_{\partial B} \frac{\partial \phi_{j}^{(\infty)}}{\partial \nu} d \sigma=-\int_{\partial B} \frac{\partial \Phi_{j}}{\partial \nu} d \sigma+c_{j} \int_{\partial B} \frac{\partial \phi}{\partial \nu} d \sigma
$$

For $n \geq 3$ we have that $\frac{\partial \phi}{\partial \nu}<0$ everywhere on $\partial B$ (by the maximum principle) and we may thus solve the above equation for $c_{j}$

$$
c_{j}=\int_{\partial B} \frac{\partial \Phi_{j}}{\partial \nu} d \sigma\left(\int_{\partial B} \frac{\partial \phi}{\partial \nu} d \sigma\right)^{-1}
$$

Let $M^{(k)}=\left\{m_{i j}^{(k)}\right\}$ denote the polarization tensor $M^{(k)}=\left(\frac{\gamma(0)}{k}-1\right) M\left(\frac{\gamma(0)}{k}\right)$ $\left(M\left(\frac{\gamma(0)}{k}\right)\right.$ being the rescaled polarization tensor introduced earlier). From (56) we get

$$
m_{i j}^{(k)}=(r-1)|B| \delta_{i j}+(r-1)^{2} \int_{\partial B} y_{i} \frac{\partial \phi_{j}^{(k)}}{\partial \nu} d \sigma_{y},
$$

with $r=\frac{\gamma(0)}{k}$. From the fact that $\frac{\partial \phi_{j}^{(k)}}{\partial \nu}$ converges uniformly to

$$
\frac{\partial \phi_{j}^{(\infty)}+}{\partial \nu}=-\frac{\partial \Phi_{j}}{\partial \nu}+c_{j} \frac{\partial \phi}{\partial \nu}
$$


we conclude that the tensor $M^{(k)}$, as $k \rightarrow \infty(r \rightarrow 0)$ converges to the tensor $M^{(\infty)}=\left\{m_{i j}^{(\infty)}\right\}$, given by

$$
\begin{aligned}
m_{i j}^{(\infty)} & =-|B| \delta_{i j}+\int_{\partial B} y_{i} \frac{\partial \phi_{j}^{(\infty)}}{\partial \nu} d \sigma_{y} \\
& =-|B| \delta_{i j}-\int_{\partial B} y_{i} \frac{\partial \Phi_{j}}{\partial \nu} d \sigma_{y}+c_{j} \int_{\partial B} y_{i} \frac{\partial \phi}{\partial \nu} d \sigma_{y} .
\end{aligned}
$$

For $n=2$ we substitute $\Phi_{i}=-y_{i}$ on $\partial B$ and use that $\frac{\partial \phi}{\partial \nu}=0$ on $\partial B$, to get

$$
m_{i j}^{(\infty)}=-|B| \delta_{i j}+\int_{\partial B} \Phi_{i} \frac{\partial \Phi_{j}}{\partial \nu} d \sigma_{y}
$$

for $n=3$ we substitute $\Phi_{i}$ for $-y_{i}$ on $\partial B$ and integrate the last (nontrivial) integral by parts, to obtain

$$
\begin{aligned}
m_{i j}^{(\infty)} & =-|B| \delta_{i j}+\int_{\partial B} \Phi_{i} \frac{\partial \Phi_{j}}{\partial \nu} d \sigma-c_{j} \int_{\partial B} \frac{\partial \Phi_{i}}{\partial \nu} d \sigma \\
& =-|B| \delta_{i j}+\int_{\partial B} \Phi_{i} \frac{\partial \Phi_{j}}{\partial \nu} d \sigma-\left(\int_{\partial B} \frac{\partial \phi}{\partial \nu} d \sigma\right)^{-1} \int_{\partial B} \frac{\partial \Phi_{i}}{\partial \nu} d \sigma \int_{\partial B} \frac{\partial \Phi_{j}}{\partial \nu} d \sigma .
\end{aligned}
$$

The latter two formulas are modulo a change of sign exactly those given for the polarization tensor in (3.8) of [7]. This means that the present asymptotic formula in the extreme case $k \rightarrow \infty$ (and for simplicity, with one inhomogeneity "centered" at the origin)

$$
\begin{aligned}
u_{\epsilon}(z) & \approx \epsilon^{n} \frac{\gamma(0)(\gamma(0)-k)}{k} \nabla_{x} N(0, z) \cdot M\left(\frac{\gamma(0)}{k}\right) \nabla_{x} U(0)+U(z) \\
& =-\epsilon^{n} \gamma(0) \nabla_{x} N(0, z) \cdot\left(-M^{(k)}\right) \nabla_{x} U(0)+U(z)
\end{aligned}
$$

is entirely consistent with the formula derived in [7] (with $\rho=1$ ). The separate proof of the asymptotic validity of this formula (for " $k=\infty$ ") is still needed since we have not shown that the term $O\left(\epsilon^{n+\frac{1}{2}}\right)$ is uniform in $k$, as $k \rightarrow \infty$.

\section{The continuous dependence of the inhomogeneities}

In a fashion completely similar to that in [7] the representation formula (54) may be used to prove asymptotic Lipschitz estimates for certain characteristics of the inhomogeneities in terms of the (rescaled) boundary data. As an example let $\omega_{\epsilon}=\cup_{i=1}^{m}\left(z_{i}+\epsilon \rho_{i} B\right)$ and $\omega_{\epsilon}^{\prime}=\cup_{i=1}^{m^{\prime}}\left(z_{i}^{\prime}+\epsilon \rho_{i}^{\prime} B\right)$ be two sets of inhomogeneities with $0<d_{0} \leq\left|z_{i}-z_{j}\right|, \forall i \neq j, 0<d_{0} \leq \operatorname{dist}\left(z_{i}, \partial \Omega\right), \forall i$, and $0<d_{0} \leq$ $\rho_{i} \leq D_{0}, \forall i$ (and similar restrictions on the $z_{i}^{\prime}$ and $\rho_{i}^{\prime}$ ). We suppose all the inhomogenieties have the same known conductivity $k \in] 0, \infty\left[\backslash\left[\gamma_{\min }, \gamma_{\max }\right]\right.$, where $\gamma_{\min }=\min _{x \in \bar{\Omega}} \gamma(x)$ and $\gamma_{\max }=\max _{x \in \bar{\Omega}} \gamma(x)$. Let $u_{\epsilon}$ and $u_{\epsilon}^{\prime}$ denote the voltage potentials corresponding to $\omega_{\epsilon}$ and $\omega_{\epsilon}^{\prime}$ for a fixed, nontrivial boundary current $\psi$. It is crucial that $\nabla U(x) \neq 0 \forall x \in \Omega$. 
Theorem 3. Suppose $n=2$ or 3 and suppose $B$ is strictly star-shaped. Let $\Gamma$ be an open, nonempty subset of $\partial \Omega$. There exist constants $0<\epsilon_{0}, \delta_{0}$ and $C$ such that if $0<\epsilon<\epsilon_{0}$ and $\epsilon^{-n}\left\|u_{\epsilon}-u_{\epsilon}^{\prime}\right\|_{L^{\infty}(\Gamma)}<\delta_{0}$ then

(i) $\quad m=m^{\prime}$, and, after appropriate reordering,

$$
\sum_{i=1}^{m}\left(\left|z_{i}-z_{i}^{\prime}\right|+\left|\rho_{i}-\rho_{i}^{\prime}\right|\right) \leq C\left(\epsilon^{-n}\left\|u_{\epsilon}-u_{\epsilon}^{\prime}\right\|_{L^{\infty}(\Gamma)}+\epsilon^{1 / 2}\right)
$$

The constants $\epsilon_{0}, \delta_{0}$ and $C$ depend on $\Gamma, \Omega, \gamma, \psi, d_{0}, D_{0}, B$, and $k$ but are otherwise independent of the two sets of inhomogeneities.

The factor $\epsilon^{-n}$ in front of $\left\|u_{\epsilon}-u_{\epsilon}^{\prime}\right\|_{L^{\infty}(\Gamma)}$ is best possible; it follows immediately from our analysis (cf. Theorem 2) that even if $\left|z_{i}-z_{i}^{\prime}\right|$ and $\left|\rho_{i}-\rho_{i}^{\prime}\right|$ are all of order 1 the difference in the boundary data is of order $\epsilon^{n}$. For fixed (small) $\epsilon$ the above theorem essentially asserts that the locations of the inhomogeneities and their relative sizes depend Lipschitz-continuously on the rescaled boundary deviation $\epsilon^{-n}|| u_{\epsilon}-u_{\epsilon}^{\prime} \|_{L^{\infty}(\Gamma)}$. We shall only here provide a sketch of the main ideas of the proof of Theorem 3; for complete details (of the proof of an almost identical result) we refer the reader to [7]. Let us also note that for $n=2$ and $\Gamma=\partial \Omega$ there is a close relation between Theorem 3 and the continuous dependence results found in [13].

Sketch of the main ideas of proof By combining the representation formula (54) with the fact that $M_{i}=\rho_{i}^{n} M\left(\gamma\left(z_{i}\right) / k\right)$, where $M(r)$ is the rescaled polarization tensor associated with $B$ and conductivity ratio $r$, we get

$$
\begin{aligned}
\epsilon^{-n}\left(u_{\epsilon}(z)\right. & \left.-u_{\epsilon}^{\prime}(z)\right)=\sum_{i=1}^{m} \rho_{i}^{n} \gamma\left(z_{i}\right) \frac{\gamma\left(z_{i}\right)-k}{k} \nabla_{x} N\left(z_{i}, z\right) \cdot M\left(\frac{\gamma\left(z_{i}\right)}{k}\right) \nabla_{x} U\left(z_{i}\right) \\
& -\sum_{i=1}^{m^{\prime}}\left(\rho_{i}^{\prime}\right)^{n} \gamma\left(z_{i}^{\prime}\right) \frac{\gamma\left(z_{i}^{\prime}\right)-k}{k} \nabla_{x} N\left(z_{i}^{\prime}, z\right) \cdot M\left(\frac{\gamma\left(z_{i}^{\prime}\right)}{k}\right) \nabla_{x} U\left(z_{i}^{\prime}\right)+O\left(\epsilon^{1 / 2}\right) .
\end{aligned}
$$

It is now not hard to see that if $\epsilon^{-n}\left\|u_{\epsilon}-u_{\epsilon}^{\prime}\right\|_{L^{\infty}(\Gamma)}$ and $\epsilon$ are both sufficiently small then $m$ and $m^{\prime}$ must necessarily be equal; for if not one may argue that there exists a function of the form

$$
F(z)=\sum_{i=1}^{m} \nabla_{x} N\left(z_{i}, z\right) \cdot \alpha_{i}-\sum_{i=1}^{m^{\prime}} \nabla_{x} N\left(z_{i}^{\prime}, z\right) \cdot \alpha_{i}^{\prime}
$$

(with $\alpha_{i} \neq 0$ and $\alpha_{i}^{\prime} \neq 0$ ) and $m \neq m^{\prime}$ such that

$$
F(z)=0 \quad z \in \Gamma \text {. }
$$

To see that $\alpha_{i}$ as well as $\alpha_{i}^{\prime}$ are not 0 we use the fact that $\nabla U$ never vanishes and that the tensor $M$ is invertible. Since $N(x, z)=N(z, x)$ we have $\nabla_{x} N\left(z_{i}, z\right)=$ $\nabla_{1} N\left(z_{i}, z\right)=\nabla_{2} N\left(z, z_{i}\right)$, where $\nabla_{1}$ and $\nabla_{2}$ indicate the gradient with respect 
to the first and second variable respectively. From the definition of $N(x, z)$ it follows that

$$
\gamma(z) \frac{\partial}{\partial \nu_{z}} \nabla_{2} N\left(z, z_{i}\right)=0 \quad z \in \partial \Omega
$$

and we thus obtain

$$
\gamma(z) \frac{\partial}{\partial \nu_{z}} F(z)=0 \quad z \in \partial \Omega
$$

The function $F$ is also easily seen to be harmonic in $\Omega \backslash\left(\left\{z_{i}\right\} \cup\left\{z_{i}^{\prime}\right\}\right)$. From the uniqueness of the solution to the Cauchy problem for the Laplacian we conclude that $F(z) \equiv 0$ in $\Omega$, but this contradicts the fact that $m \neq m^{\prime}$ (since the points in the set $\left\{z_{i}\right\}$ as well as in the set $\left\{z_{i}^{\prime}\right\}$ are mutually distinct and since all the vectors $\alpha_{i}$ and $\alpha_{i}^{\prime}$ are different from zero).

When it comes to "verifying" ( $i i)$ consider for simplicity the case when $\gamma(x)=$ $\gamma$ (a constant), and suppose also that $U(x)=x_{1}$ (corresponding to boundary current $\left.\psi=\gamma \nu_{1}\right)$. Then

$$
\begin{aligned}
& \epsilon^{-n}\left(u_{\epsilon}(z)-u_{\epsilon}^{\prime}(z)\right) \\
& \quad=\frac{\gamma(\gamma-k)}{k} \sum_{i=1}^{m}\left[\rho_{i}^{n} \nabla_{x} N\left(z_{i}, z\right)-\left(\rho_{i}^{\prime}\right)^{n} \nabla_{x} N\left(z_{i}^{\prime}, z\right)\right] \cdot \mu_{1}+O\left(\epsilon^{1 / 2}\right)
\end{aligned}
$$

$\mu_{1}$ being the first column of $M(\gamma / k)$. A simple calculation gives

$$
\begin{aligned}
\sum & {\left[\rho_{i}^{n} \nabla_{x} N\left(z_{i}, z\right)-\left(\rho_{i}^{\prime}\right)^{n} \nabla_{x} N\left(z_{i}^{\prime}, z\right)\right] \cdot \mu_{1} } \\
= & \sum\left[\left(\rho_{i}-\rho_{i}^{\prime}\right) n\left(\bar{\rho}_{i}\right)^{n-1} \nabla_{x} N\left(z_{i}, z\right) \cdot \mu_{1}+\left(\rho_{i}^{\prime}\right)^{n}\left(\nabla_{x} N\left(z_{i}, z\right)-\nabla_{x} N\left(z_{i}^{\prime}, z\right)\right)\right] \cdot \mu_{1} \\
= & \sum\left(\left|\rho_{i}-\rho_{i}^{\prime}\right|+\left|z_{i}-z_{i}^{\prime}\right|\right) \\
& \quad \times \sum\left[d \rho_{i} n\left(\bar{\rho}_{i}\right)^{n-1} \nabla_{x} N\left(z_{i}, z\right) \cdot \mu_{1}+\left(\rho_{i}^{\prime}\right)^{n}<d z_{i}, D_{x}^{2} N\left(\bar{z}_{i}, z\right) \mu_{1}>\right],
\end{aligned}
$$

with $\sum\left(\left|d \rho_{i}\right|+\left|d z_{i}\right|\right)=1$ for some $\bar{\rho}_{i}(z)$ and $\bar{z}_{i}(z)$ ("between" $\rho_{i}$ and $\rho_{i}^{\prime}$ and $z_{i}$ and $z_{i}^{\prime}$ respectively). By a fairly simple argument it follows from (73) that we either (with appropriate reordering) have the desired continuous dependence estimate

$$
\sum_{i=1}^{m}\left(\left|\rho_{i}-\rho_{i}^{\prime}\right|+\left|z_{i}-z_{i}^{\prime}\right|\right) \leq C\left(\epsilon^{-n}|| u_{\epsilon}-u_{\epsilon}^{\prime} \|_{L^{\infty}(\Gamma)}+\epsilon^{1 / 2}\right)
$$

or there exist (infinitessimal) perturbations $d \rho_{i}$ and $d z_{i}$ (with $\sum_{i=1}^{m}\left(\left|d \rho_{i}\right|+\right.$ $\left.\left.\left|d z_{i}\right|\right)=1\right)$, points $z_{i}\left(=z_{i}^{\prime}=\bar{z}_{i}\right)$ and dilatation parameters $\rho_{i}\left(=\rho_{i}^{\prime}=\bar{\rho}_{i}\right)$ so that

$$
G(z)=\sum_{i=1}^{m}\left[d \rho_{i} n\left(\rho_{i}\right)^{n-1} \nabla_{x} N\left(z_{i}, z\right) \cdot \mu_{1}+\left(\rho_{i}\right)^{n}<d z_{i}, D_{x}^{2} N\left(z_{i}, z\right) \mu_{1}>\right]=0
$$

for $z \in \Gamma$. Just as was the case with $F$, the function $G$ has a vanishing normal derivative on $\partial \Omega$ and it is harmonic in $\Omega$ except at the points $\left\{z_{i}\right\}$ and $\left\{z_{i}^{\prime}\right\}$.If $G(z)=0, z \in \Gamma$, it would now follow from the unique continuation property of 
harmonic functions that $G(z) \equiv 0$ in $\Omega$. Since the points $z_{i}$ are mutually different and since the dilatation parameters $\rho_{i}$ and the vector $\mu_{1}$ are nonzero, the function $G$ can only identically vanish if $d \rho_{i}=d z_{i}=0, i=1 \ldots m$. This, however, would be a contradiction to the fact that $\sum\left(\left|d \rho_{i}\right|+\left|d z_{i}\right|\right)=1$. We therefore conclude that the desired continuous dependence estimate holds.

For $n=2$ we could prove a similar continuous dependence estimate for the $Q_{i}$ (the angles of rotations) and the "centers" $z_{i}$, provided $M(r)$ is not isotropic. It is, however, not possible to prove such an estimate for the dilatation parameters, the angles of rotations and the "centers" simultaneously (see [7]). To conclude this section consider the case of two sets of inhomogeneities $\omega_{\epsilon}=\cup_{i=1}^{m}\left(z_{i}+\epsilon \rho_{i} B\right)$ and $\omega_{\epsilon}^{\prime}=\cup_{i=1}^{m}\left(z_{i}+\epsilon \rho_{i}^{\prime} B\right)$ with $B=$ the unit ball (centered at the origin).Suppose the inhomogeneity $z_{i}+\epsilon \rho_{i} B$ has conductivity $k_{i}$ and the inhomogeneity $z_{i}+\epsilon \rho_{i}^{\prime} B$ has conductivity $k_{i}^{\prime}$. Notice that the number of inhomogeneities and their centers are the same for $\omega_{\epsilon}$ and $\omega_{\epsilon}^{\prime}$. Suppose $\gamma(x)=\gamma$ (a constant) and $U(x)=x_{1}$. In this case

$$
\begin{aligned}
u_{\epsilon}(z) & -u_{\epsilon}^{\prime}(z) \\
= & \epsilon^{n} \omega_{n} \gamma \sum_{i=1}^{m} \nabla_{x} N\left(z_{i}, z\right) \cdot e_{1}\left[\frac{\left(\gamma-k_{i}\right) \rho_{i}^{n}}{(n-1) \gamma+k_{i}}-\frac{\left(\gamma-k_{i}^{\prime}\right)\left(\rho_{i}^{\prime}\right)^{n}}{(n-1) \gamma+k_{i}^{\prime}}\right]+O\left(\epsilon^{n+\frac{1}{2}}\right) .
\end{aligned}
$$

It is clear from this expression that given a set of dilatation parameters $\rho_{i}$ and conductivities $k_{i}$ we can chose any other set of dilatation parameters $\rho_{i}^{\prime}>\rho_{i}$ and then determine conductivities $k_{i}^{\prime}$ so that $u_{\epsilon}-u_{\epsilon}^{\prime}$ is of order $\epsilon^{n+\frac{1}{2}}$ on $\partial \Omega$. Similarly given a set of dilatation parameters $\rho_{i}$ and conductivities $k_{i}$ we can chose any other set of conductivities $k_{i}^{\prime}$, with $k_{i}^{\prime}$ being on the same side of $\gamma$ as $k_{i}$, and then we can determine dilatation parameters $\rho_{i}^{\prime}$ so that $u_{\epsilon}-u_{\epsilon}^{\prime}$ is of order $\epsilon^{n+\frac{1}{2}}$ on $\partial \Omega$. In either case $\epsilon^{-n}|| u_{\epsilon}-u_{\epsilon}^{\prime} \|_{L^{\infty}(\Gamma)}+\epsilon^{1 / 2}$ is of order $\epsilon^{1 / 2}$, but $\sum\left(\left|\rho_{i}-\rho_{i}^{\prime}\right|+\left|k_{i}-k_{i}^{\prime}\right|\right)$ is of order 1 . Therefore there is no Lipschitz estimate like that in Theorem 3 , involving the $\rho^{\prime} s$ and the $k^{\prime} s$. Equivalently, in terms of the inverse problem, it is not possible to determine the $\epsilon \rho_{i}^{\prime} s$ and the $k_{i}^{\prime} s$ simultaneously from knowledge of the lowest order term in $u_{\epsilon}-U$.

\section{Computational results}

In this section we describe some computational experiments concerning the formula (55). These experiments quite clearly demonstrate the viability of this formula as an effective tool for the identification of well separated, internal inhomogeneities inside an otherwise uniform conductor.

The constant background conductivity $\gamma(x)=\gamma$ is in all our actual computations chosen to be 1 . We always use the background voltage potential $U(x)=x_{1}$, corresponding to the boundary current $\psi=\gamma \nu_{1}\left(=\nu_{1}\right)$. To apply the formula (55) we need to calculate the tensors $M_{i}$. This is in each case done by calculating 
approximations to the functions $\phi_{j}$ (on $\partial B$ ) and then employing the formula (58) to calculate the individual entries of $M$. We also need the boundary data $\left(\left.u_{\epsilon}\right|_{\partial \Omega}\right)$; in practice this data would be measured, but here we generate it numerically (so called synthetic data). Approximations to the functions $\left.\phi_{j}\right|_{\partial B}$ and $\left.u_{\epsilon}\right|_{\partial \Omega}$ are calculated by discretizations of integral equation formulations. Since the calculation of an approximation to $\left.u_{\epsilon}\right|_{\partial \Omega}$ is very similar to the calculation of approximations to the $\left.\phi_{j}\right|_{\partial B}$, we describe in some detail how the latter approximations are arrived at, and then we very briefly discuss the main differences concerning $u_{\epsilon}$. In all our computational work we restrict attention to the two-dimensional case.

Recall, that $\phi_{j}$ is the solution to the boundary value problem (52). We note that, as usual, a superscript of + and - will signify the values of the particular function approaching $\partial B$ from the exterior and interior of $B$ respectively. Let $\Phi$ denote the fundamental solution

$$
\Phi(x, y)=-\frac{1}{2 \pi} \log |x-y|,
$$

( $\Phi$ is as before, only with $\gamma=1$ ). Let $x \in \Re^{2} \backslash \bar{B}$ and let $S_{R}$ be a disk of radius $R$, with $R$ sufficiently large that $\bar{B} \subset S_{R}$ and $x \in S_{R}$. Applying Greens formula for the domain $S_{R} \backslash \bar{B}$ we obtain

$$
\begin{aligned}
\phi_{j}(x)= & \int_{\partial S_{R}} \frac{\partial \phi_{j}}{\partial \nu_{y}}(y) \Phi(x, y) d \sigma_{y}-\int_{\partial S_{R}} \phi_{j}(y) \frac{\partial \Phi(x, y)}{\partial \nu_{y}} d \sigma_{y} \\
& -\int_{\partial B}{\frac{\partial \phi_{j}}{\partial \nu_{y}}}^{+}(y) \Phi(x, y) d \sigma_{y}+\int_{\partial B} \phi_{j}(y) \frac{\partial \Phi(x, y)}{\partial \nu_{y}} d \sigma_{y} \quad x \in S_{R} \backslash \bar{B} .
\end{aligned}
$$

Here the unit normal, $\nu$, is directed towards the exterior of $S_{R}$ along $\partial S_{R}$, and towards the exterior of B along $\partial B$. Recall that for $|y| \rightarrow \infty$ we have $\phi_{j}(y)=$ $O\left(|y|^{-1}\right)$ and $\nabla \phi_{j}(y)=O\left(|y|^{-2}\right)$. Thus

$$
\lim _{R \rightarrow \infty} \int_{\partial S_{R}} \frac{\partial \phi_{j}}{\partial \nu_{y}}(y) \Phi(x, y) d \sigma_{y}=0
$$

and

$$
\lim _{R \rightarrow \infty} \int_{\partial S_{R}} \phi_{j}(y) \frac{\partial \Phi(x, y)}{\partial \nu_{y}} d \sigma_{y}=0
$$

As $R \rightarrow \infty$ in (74) we now get

$$
\phi_{j}(x)=\int_{\partial B} \phi_{j}(y) \frac{\partial \Phi(x, y)}{\partial \nu_{y}} d \sigma_{y}-\int_{\partial B}{\frac{\partial \phi_{j}}{\partial \nu_{y}}}^{+}(y) \Phi(x, y) d \sigma_{y} \quad x \in \Re^{n} \backslash \bar{B} .
$$

Using the boundary condition $-\frac{\gamma}{k} \frac{\partial \phi_{j}}{\partial \nu_{y}}{ }^{+}+{\frac{\partial \phi_{j}}{\partial \nu_{y}}}^{-}=\nu_{j}$ on $\partial B$ and the integral identity $\int_{\partial B} \frac{\partial \phi_{j}}{\partial \nu_{y}}(y) \Phi(x, y) d \sigma_{y}=\int_{\partial B} \phi_{j}(y) \frac{\partial \Phi}{\partial \nu_{y}}(x, y) d \sigma_{y}, x \in \Re^{2} \backslash \bar{B}$, we calculate

$\phi_{j}(x)=\int_{\partial B} \phi_{j}(y) \frac{\partial \Phi(x, y)}{\partial \nu_{y}} d \sigma_{y}-\frac{k}{\gamma} \int_{\partial B} \frac{\partial{\phi_{j}}^{-}}{\partial \nu_{y}}(y) \Phi(x, y) d \sigma_{y}+\frac{k}{\gamma} \int_{\partial B} \nu_{j} \Phi(x, y) d \sigma_{y}$ 


$$
\begin{aligned}
& =\int_{\partial B} \phi_{j}(y) \frac{\partial \Phi(x, y)}{\partial \nu_{y}} d \sigma_{y}-\frac{k}{\gamma} \int_{\partial B} \phi_{j}(y) \frac{\partial \Phi(x, y)}{\partial \nu_{y}} d \sigma_{y}+\frac{k}{\gamma} \int_{\partial B} \nu_{j} \Phi(x, y) d \sigma_{y} \\
& =\frac{\gamma-k}{\gamma} \int_{\partial B} \phi_{j}(y) \frac{\partial \Phi(x, y)}{\partial \nu_{y}} d \sigma_{y}+\frac{k}{\gamma} \int_{\partial B} \nu_{j} \Phi(x, y) d \sigma_{y} .
\end{aligned}
$$

In the limit as $x \rightarrow \partial B$, this yields

$$
\phi_{j}(x)=\frac{\gamma-k}{\gamma} \int_{\partial B} \phi_{j}(y) \frac{\partial \Phi(x, y)}{\partial \nu_{y}} d \sigma_{y}+\frac{\gamma-k}{2 \gamma} \phi_{j}(x)+\frac{k}{\gamma} \int_{\partial B} \nu_{j} \Phi(x, y) d \sigma_{y}
$$

where the second term in the right hand side reflects the jump associated with the double layer potential. Rearranging terms and inserting the expression for $\Phi(x, y)$ we finally arrive at the integral equation

$$
\phi_{j}(x)+\frac{(k-\gamma)}{\pi(k+\gamma)} \int_{\partial B} \phi_{j}(y) \frac{(x-y) \cdot \nu}{|x-y|^{2}} d \sigma_{y}=-\frac{k}{\pi(k+\gamma)} \int_{\partial B} \nu_{j} \log |x-y| d \sigma_{y},
$$

for $x \in \partial B$. Equivalently, $\phi_{j}$ is the solution to

$$
\phi_{j}+A \phi_{j}=f_{j}
$$

with

$$
\left(A \phi_{j}\right)(x)=\frac{k-\gamma}{\pi(k+\gamma)} \int_{\partial B} \phi_{j}(y) \frac{(x-y) \cdot \nu}{|x-y|^{2}} d \sigma_{y}
$$

and

$$
f_{j}(x)=-\frac{k}{\pi(k+\gamma)} \int_{\partial B} \nu_{j} \log |x-y| d \sigma_{y} .
$$

We use a collocation method based on trigonometric interpolation for approximating the solution to (75). That is, we seek an approximate solution for $\phi_{j}$ on $\partial B$ from the finite dimensional subspace $T_{p}=\operatorname{span}\left\{\xi_{l}\right\}_{l=1}^{p}$, generated by the first $p$ functions of the family $\{1, \cos t, \sin t, \cos 2 t, \sin 2 t, \ldots\}$. We require that the system of equations be satisfied only at a finite number of collocation points, $\left\{x_{1}, x_{2}, \ldots, x_{p}\right\}$, along $\partial B$. To be more precise, the approximation to $\phi_{j}$ is expressed as a linear combination $\phi_{j}^{(p)}=\sum_{l=1}^{p} c_{j}^{(l)} \xi_{l}$, where the coefficients $c_{j}^{(l)}$ are determined so that $\phi_{j}^{(p)}$ satisfies

$$
\phi_{j}^{(p)}\left(x_{i}\right)+A \phi_{j}^{(p)}\left(x_{i}\right)=f_{j}\left(x_{i}\right) \quad i=1, \ldots, p
$$

The collocation points $x_{1}, \ldots, x_{p}$ are chosen so that the subspace $T_{p}$ is unisolvent with respect to these points. Inserting $\phi_{j}^{(p)}=\sum_{l=1}^{p} c_{j}^{(l)} \xi_{l}$ into equation (76) we get

$$
\sum_{l=1}^{p} c_{j}^{(l)} \xi_{l}\left(x_{i}\right)+\sum_{l=1}^{p}\left(A \xi_{l}\right)\left(x_{i}\right) c_{j}^{(l)}=f_{j}\left(x_{i}\right) \quad i=1, \ldots, p
$$

or equivalently

$$
S \hat{c}_{j}=\hat{f}_{j}
$$


where $\hat{c}_{j}$ is the coefficient vector $\hat{c}_{j}=\left(c_{j}^{(1)}, \ldots, c_{j}^{(p)}\right)^{T}$, and the entries in the matrix, $S=\left\{s_{i l}\right\}$, and the right hand side, $\hat{f}_{j}=\left\{\hat{f}_{j}^{(i)}\right\}$, are given by

$$
\begin{gathered}
s_{i l}=\xi_{l}\left(x_{i}\right)+\left(A \xi_{l}\right)\left(x_{i}\right), \\
\hat{f}_{j}^{(i)}=f_{j}\left(x_{i}\right) .
\end{gathered}
$$

The integral equation for $u_{\epsilon}$ is, as mentioned earlier, derived in a totally similar fashion. There will now be equations corresponding to the boundaries of all the inhomogeneities as well as to the outer boundary. We again use a trigonometric collocation method to find an approximate solution. We take the same number of collocation points for each inhomogeneity, this number being somewhat smaller than the number of collocation points on the outer boundary. Typically we take $25-50$ points for each inhomogeneity and 50-100 points on the outer boundary.

Having calculated the rescaled polarization tensors and the synthetic data we use the formula (55) to identify the inhomogeneities. In the two dimensional case, with the expression $-\frac{1}{2 \pi \gamma} \log |x-y|$ inserted in place of $\Phi(x, y)$, this formula reads

$$
\begin{aligned}
u_{\epsilon}(z) & -U(z)+\frac{1}{2 \pi} \int_{\partial \Omega}\left(u_{\epsilon}(x)-U(x)\right) \frac{(z-x) \cdot \nu_{x}}{|x-z|^{2}} d \sigma_{x} \\
= & \epsilon^{2} \frac{1}{2 \pi} \sum_{i=1}^{m} \frac{\gamma-k_{i}}{k_{i}} \frac{\left(z-z_{i}\right) \cdot \nu_{x}}{\left|z_{i}-z\right|^{2}} \cdot M_{i} \nabla_{x} U\left(z_{i}\right)+O\left(\epsilon^{2+\frac{1}{2}}\right) .
\end{aligned}
$$

The left hand side $L\left(u_{\epsilon}-U\right)(z)=u_{\epsilon}(z)-U(z)+\frac{1}{2 \pi} \int_{\partial \Omega}\left(u_{\epsilon}(x)-U(x)\right) \frac{(z-x) \cdot \nu_{x}}{|x-z|^{2}} d \sigma_{x}$ is entirely known on $\partial \Omega$ once $u_{\epsilon}-U$ is known on $\partial \Omega$. The sum on the right hand side $\Sigma(z)=\epsilon^{2} \frac{1}{2 \pi} \sum_{i=1}^{m} \frac{\gamma-k_{i}}{k_{i}} \frac{\left(z-z_{i}\right) \cdot \nu_{x}}{\left|z_{i}-z\right|^{2}} \cdot M_{i} \nabla_{x} U\left(z_{i}\right)$ is a fairly explicit function of the location and certain other features of the inhomogeneities. Our computational identification algorithm consists in minimizing a discreet $L^{2}$ norm of the residual $L\left(u_{\epsilon}-U\right)-\Sigma$ on $\partial \Omega$. We choose the boundary expression $L\left(u_{\epsilon}-U\right)-\Sigma=$ $L\left(u_{\epsilon}-U-L^{-1} \Sigma\right)$ from $(55)$ over the essentially equivalent boundary expression $u_{\epsilon}-U-\tilde{\Sigma}$ from (54) because of its explicitness and simplicity. It is clear that both expressions are based on finding the second term in an asymptotic expansion of $u_{\epsilon}$ in powers of $\epsilon^{n}$. In the calculations presented here we restrict our attention to inhomogeneities, $z_{i}+\epsilon \rho_{i} Q_{i} B$, which are dilatations, rotations and translations of one common domain, and the conductivities of which are all the same. In this case $M_{i}=\rho_{i}^{2} Q_{i} M Q_{i}^{T}$ where $\rho_{i}>0$ are dilatation parameters, $Q_{i}$ are rotations and $M$ is the rescaled polarization tensor corresponding to the common domain, $B$, and the common conductivity ratio $\gamma / k$. We take $U$ to be the special solution $U(x)=x_{1}$, corresponding to the boundary current $\psi=\gamma \nu_{1}\left(=\nu_{1}\right)$. The domain $\Omega$ is taken to be the disk of radius 10 , centered at the origin, and we shall here only consider inhomogeneities that are shaped as disks or ellipsoids (even though we have performed experiments with other shapes). We select $J$ equidistant points, 
$y_{1}, \ldots, y_{J}$, on $\partial \Omega$ (typically $J=50$ ) and we seek the unknown parameters of the inhomogeneities as the solution to the nonlinear least squares problem

$$
\begin{aligned}
\min \sum_{j=1}^{J}[ & u_{\epsilon}\left(y_{j}\right)-U\left(y_{j}\right)+\frac{1}{2 \pi} \int_{\partial \Omega}\left(u_{\epsilon}(x)-U(x)\right) \frac{\left(y_{j}-x\right) \cdot \nu_{x}}{\left|x-y_{j}\right|^{2}} d \sigma_{x} \\
& \left.-\frac{1}{2 \pi} \sum_{i=1}^{m}\left(\epsilon \rho_{i}\right)^{2} \frac{(\gamma-k)}{k} \frac{y_{j}-z_{i}}{\left|z_{i}-y_{j}\right|^{2}} \cdot Q_{i} M_{i} Q_{i}^{T} e_{1}\right]^{2}
\end{aligned}
$$

To calculate the inside, $L\left(u_{\epsilon}-U\right)-\Sigma$, of the least squares functional, we use the equivalent formula

$$
\begin{array}{r}
L\left(u_{\epsilon}-U\right)(z)-\Sigma(z)=u_{\epsilon}(z)-\frac{1}{2 \pi}\left(\sum_{i=1}^{m}\left(\epsilon \rho_{i}\right)^{2} \frac{(\gamma-k)}{k} \frac{z-z_{i}}{\left|z_{i}-z\right|^{2}} \cdot Q_{i} M_{i} Q_{i}^{T} e_{1}\right. \\
\left.-\int_{\partial \Omega} \nu_{1} \log |x-z| d \sigma_{x}-\int_{\partial \Omega} u_{\epsilon} \frac{(z-x) \cdot \nu}{|x-z|^{2}} d \sigma_{x}\right) .
\end{array}
$$

We minimize over $\left\{z_{i}, \epsilon \rho_{i}\right\}$ when the orientations are known or when they are irrelevant, as is the case when the inhomogeneities are all disks, and we minimize over $\left\{z_{i}, Q_{i}\right\}$ when the dilatation parameters are known. When all the parameters are unknown one can attempt to minimize over $\left\{z_{i}, \epsilon \rho_{i}, Q_{i}\right\}$, however, as pointed out earlier, there may be considerable non-uniqueness of the minimizer in that case. The approximate formula $L\left(u_{\epsilon}-U\right) \approx \Sigma$ on which our identification algorithm is based has been verified to hold asymptotically as $\epsilon \rightarrow 0$. Before we proceed to describe any of our identification experiments we shall try to assess the practical validity of this formula.

We consider a single inhomogeneity of the form $z_{1}+\epsilon \rho_{1} B$, having conductivity $k=10$, and compare the graph of the function

$$
u_{\epsilon}(z)-U(z), \quad z \in \partial \Omega
$$

to the graph of

$$
\begin{aligned}
& L\left(u_{\epsilon}-U\right)(z)-\Sigma(z) \\
& =u_{\epsilon}(z)-U(z)+\frac{1}{2 \pi} \int_{\partial \Omega}\left(u_{\epsilon}(x)-U(x)\right) \frac{(z-x) \cdot \nu_{x}}{|x-z|^{2}} d \sigma_{x} \\
& \quad-\left(\epsilon \rho_{1}\right)^{2} \frac{1}{2 \pi} \frac{\gamma-k}{k} \frac{\left(z-z_{1}\right) \cdot \nu_{x}}{\left|z_{1}-z\right|^{2}} \cdot M \nabla_{x} U\left(z_{1}\right), \quad z \in \partial \Omega .
\end{aligned}
$$

The reference conductivity is, as always, taken to be $\gamma=1$. Figure 1(a) and (b) displays the graph of these two functions for a circular inclusion with center $z_{1}=(0,6)$ and radius $\epsilon \rho_{1}=1.00$ (in this case $B$ is the unit disk). In figure 2 (a) and (b) we graph the results obtained for an elliptical shaped inhomogeneity having eccentricity ratio $1: 2$, center of mass $z_{1}=(0,6)$, and scaling factor $\epsilon \rho_{1}=$ 0.50 . In this case $B$ is an ellipse with major axis 4 and minor axis 2 ; the major 


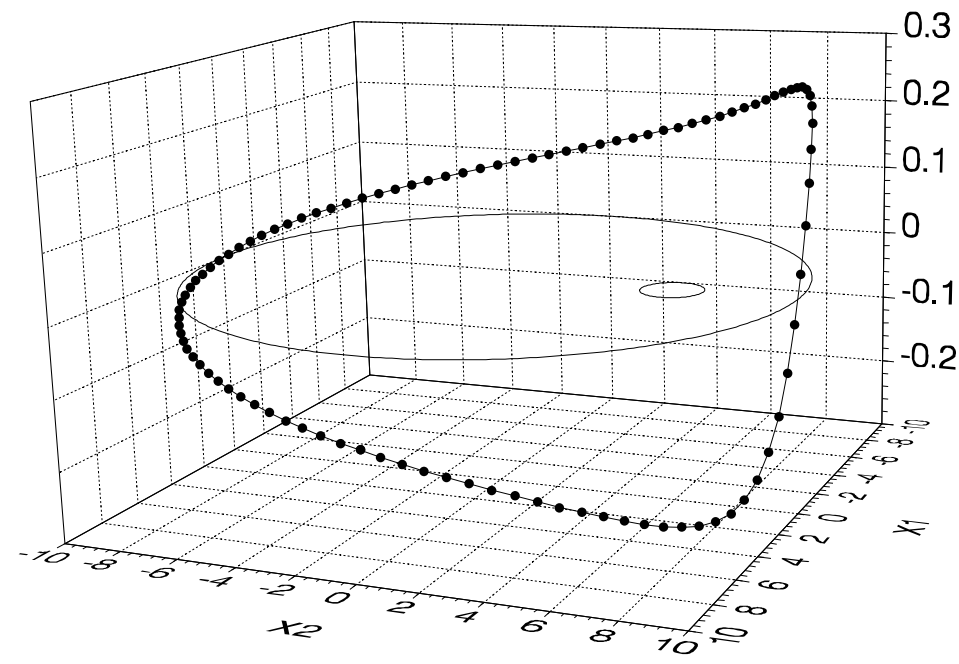

(a) $u_{\epsilon}-U$

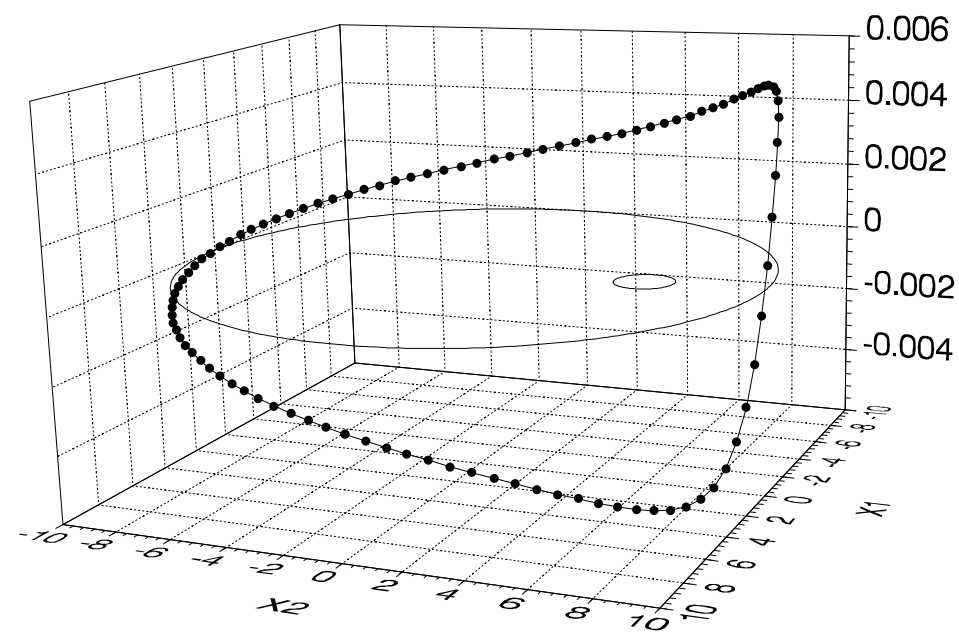

(b) $L\left(u_{\epsilon}-U\right)-\Sigma$

Figure 1: Remainder terms for a circular inhomogeneity 


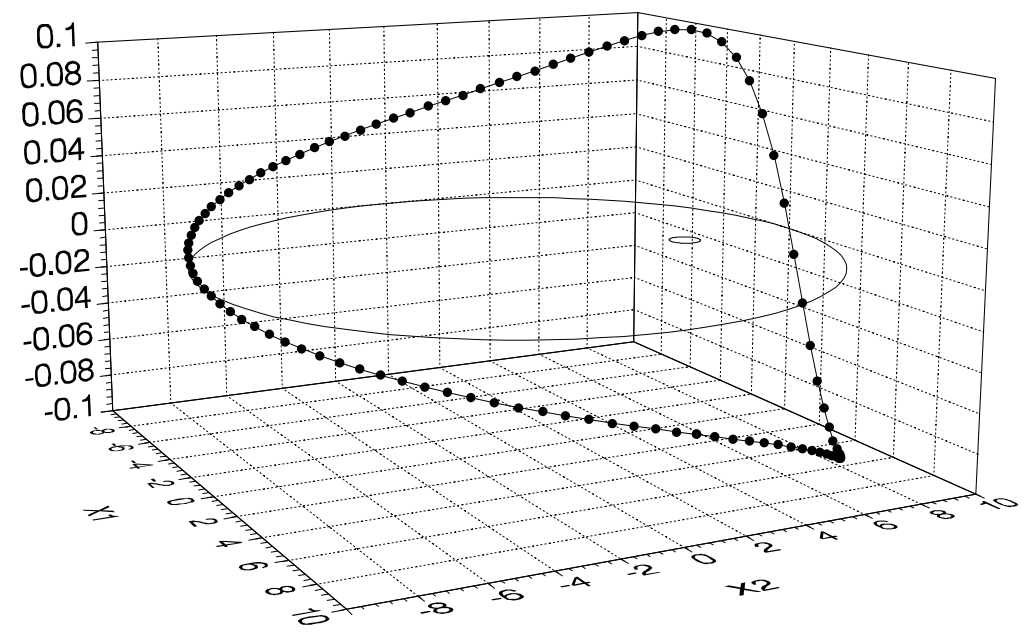

(a) $u_{\epsilon}-U$

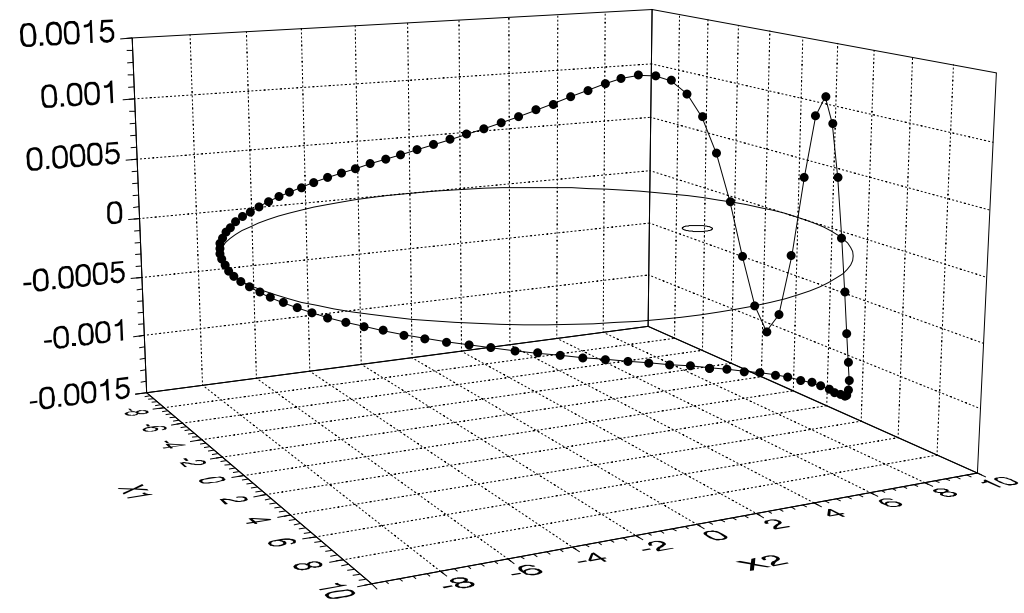

(b) $L\left(u_{\epsilon}-U\right)-\Sigma$

Figure 2: Remainder terms for an elliptical inhomogeneity 
axis is parallel to the $x_{2}$ axis. The graphs obtained in figures 1 and 2 were based on evaluating (79) and (80) at 100 uniformly spaced points along $\partial \Omega$. As anticipated, the remainder $L\left(u_{\epsilon}-U\right)-\Sigma$ is much smaller than the remainder $u_{\epsilon}-U$. In both cases the largest values are on that part of the boundary which is nearest to the inhomogeneity. The two graphs are approximately of the same shape for the disk, whereas their shapes differ for the ellipse.

Our theoretical result estimate the term $L\left(u_{\epsilon}-U\right)-\Sigma$ to be of the order $\epsilon^{n+\frac{1}{2}}=\epsilon^{2.5}$, however, this is far too conservative; one should expect it to be of order $\epsilon^{2 n}=\epsilon^{4}$, since it largely represents the "quadratic" term when $L\left(u_{\epsilon}-U\right)$ is expanded in a power series in the volume of the inhomogeneity. To demonstrate this point, we have calculated a discrete $L^{2}$ norm of the term $L\left(u_{\epsilon}-U\right)-\Sigma$ correponding to four different cases of a circular inhomogeneity centered at $(0,6)$ with conductivity $k=10$. For the $l^{2}$ norm we used 25 evenly spaced points along $\partial \Omega$. The four inhomogeneities have the form $(0,6)+\epsilon \rho_{1} B$ with $B$ being the unit disk and the scaling factor $\epsilon \rho_{1}$ taking the values $\epsilon \rho_{1}=.5,1,2$, and 3 . The graph of the $l^{2}$ norm, $E$, as a function of the parameter $\epsilon \rho_{1}$ is displayed on a log-log plot in figure 3(a). Geometric regression analysis shows the correlation coefficient for the data to be 0.919 and produces the "best fit"

$$
E \approx 0.0141\left(\epsilon \rho_{1}\right)^{3.95}
$$

We have repeated this experiment for four different inhomogeneities of the form $(0,6)+\epsilon \rho_{1} B$, where $B$ is the elliptical domain centered at the origin with major axis 4 (parallel to the $x_{2}$-axis) and minor axis 2 . The $l^{2}$ norm of the remainder, based on 25 evenly spaced points along $\partial \Omega$, was calculated for the values $\epsilon \rho_{1}=$ $0.25,0.50,1.00$, and 1.50. The corresponding graph is shown in figure $3(\mathrm{~b})$. The correlation coefficient for this data is calculated to be 0.914 and geometric regression analysis produces the "best fit"

$$
E \approx 0.0435\left(\epsilon \rho_{1}\right)^{4.01}
$$

We next study the remainder terms for a conductor containing a single inhomogeneity, as we vary the conductivity, $k$, of the inhomogeneity. Specifically, we examine the two norms

$$
\text { (a) }\left\|u_{\epsilon}-U\right\|_{L^{\infty}(\partial \Omega)} \text { and (b) }\left\|L\left(u_{\epsilon}-U\right)-\Sigma\right\|_{L^{\infty}(\partial \Omega)},
$$

for conductivities satisfying $.00001<k<100000$. The inhomogeneity is a unit disk centered at $(0,6)$. The results are displayed in figure 4 . As expected, both norms approach 0 as the conductivity on the inhomogeneity approaches the reference conductivity, $\gamma=1$. Figure 5 provides a comparison of the two remainders. Here we have plotted $\left\|L\left(u_{\epsilon}-U\right)-\Sigma\right\|_{L^{\infty}(\partial \Omega)}$ against $\left\|u_{\epsilon}-U\right\|_{L^{\infty}(\partial \Omega)}$ on a loglog scale. In plots 5 (a) and (b) we display the results for conductivity values $k<1$ and $k>1$ respectively. Geometric regression analysis shows the correlation 


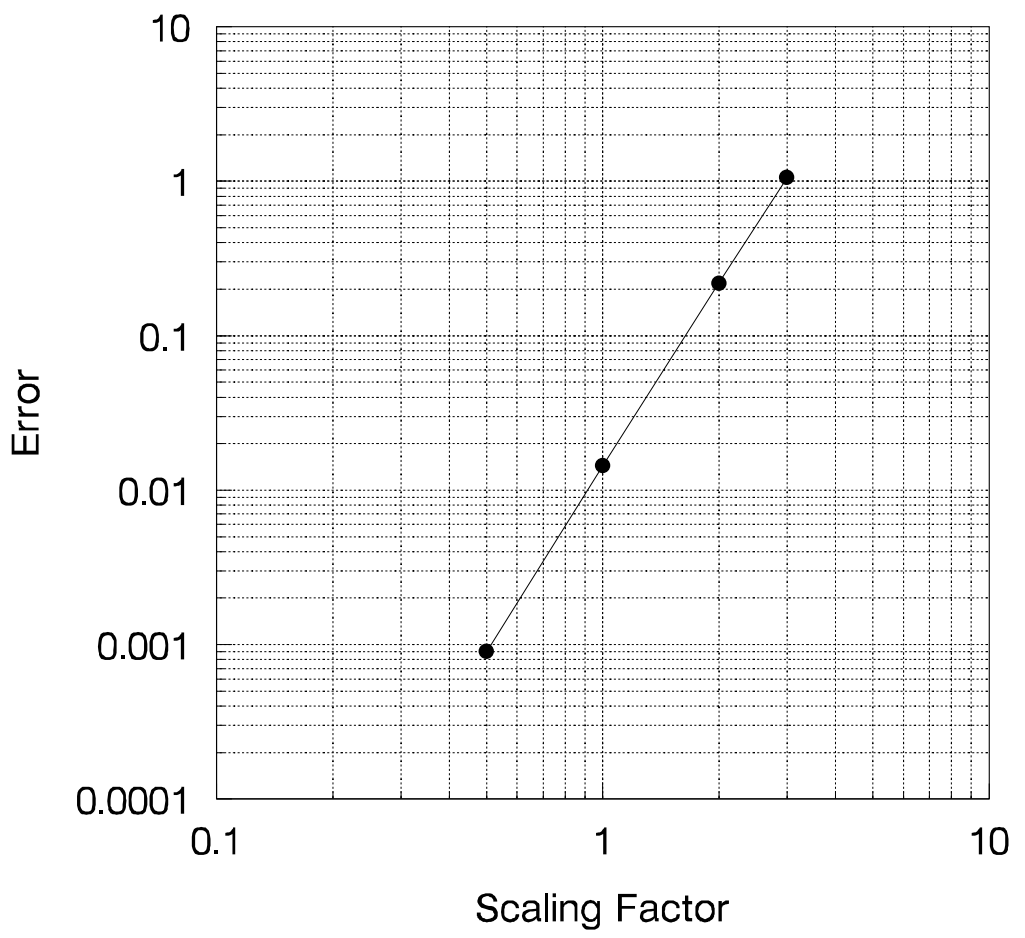

(a) Plot for circular inhomogeneities

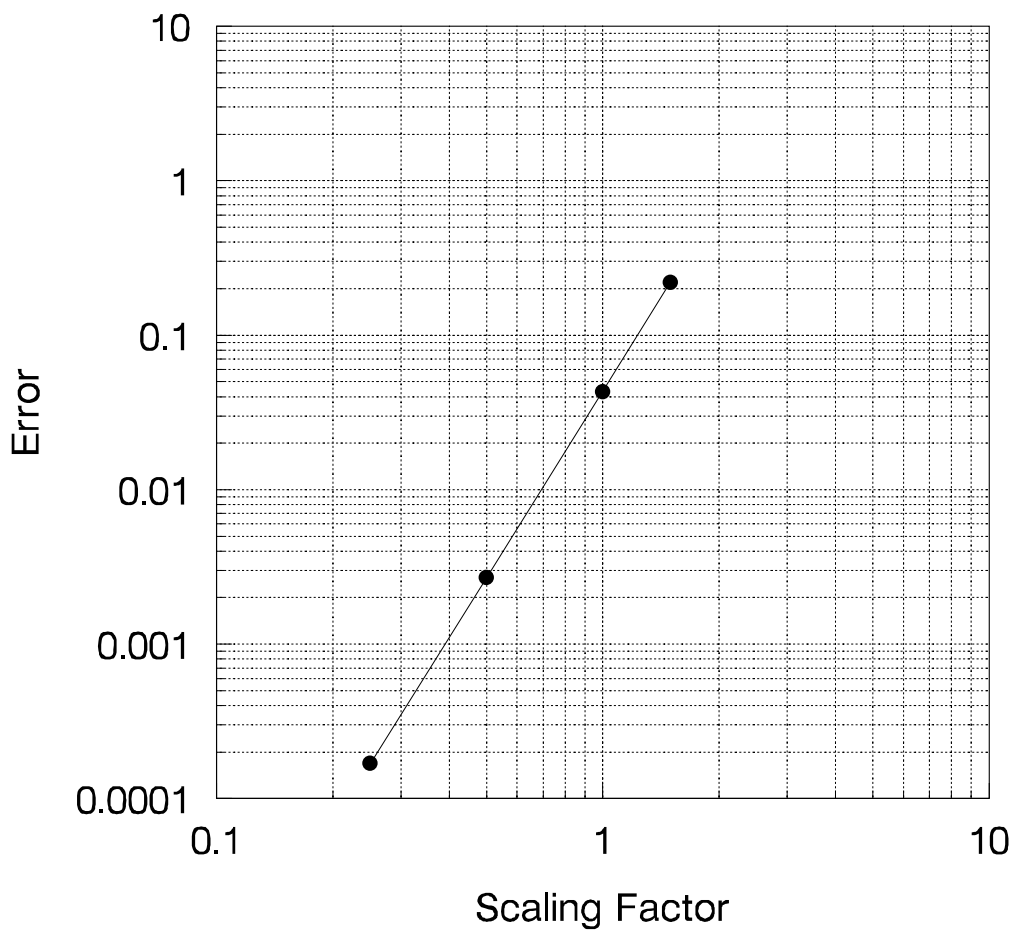

(b) Plot for elliptical inhomogeneities

Figure 3: Log-log plots of the remainder (error) vs the scaling factor, $\epsilon \rho_{1}$. 


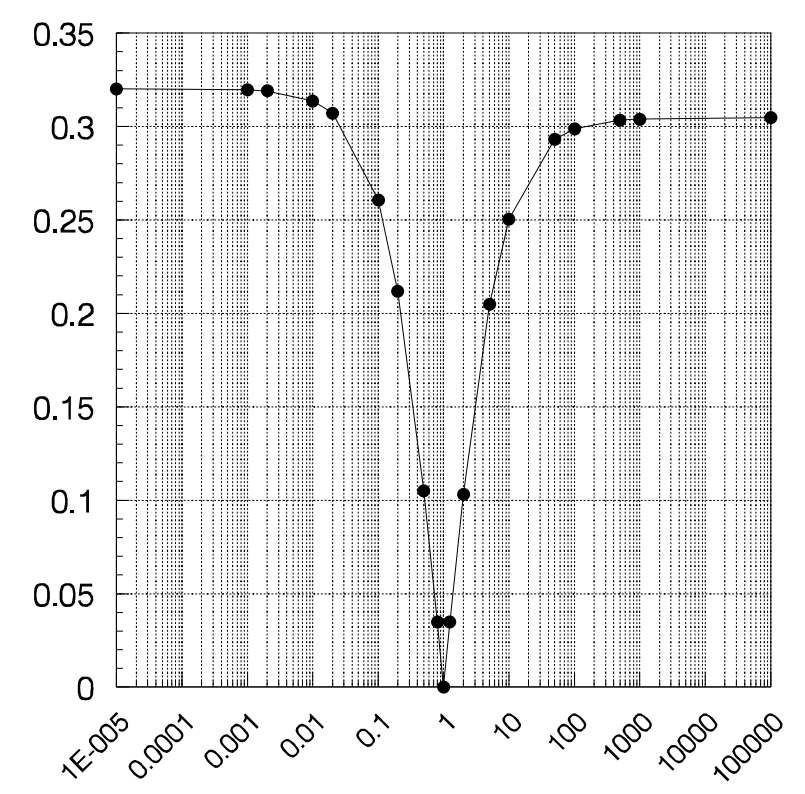

(a) $k$ vs. $\left\|u_{\epsilon}-U\right\|_{\infty}$.

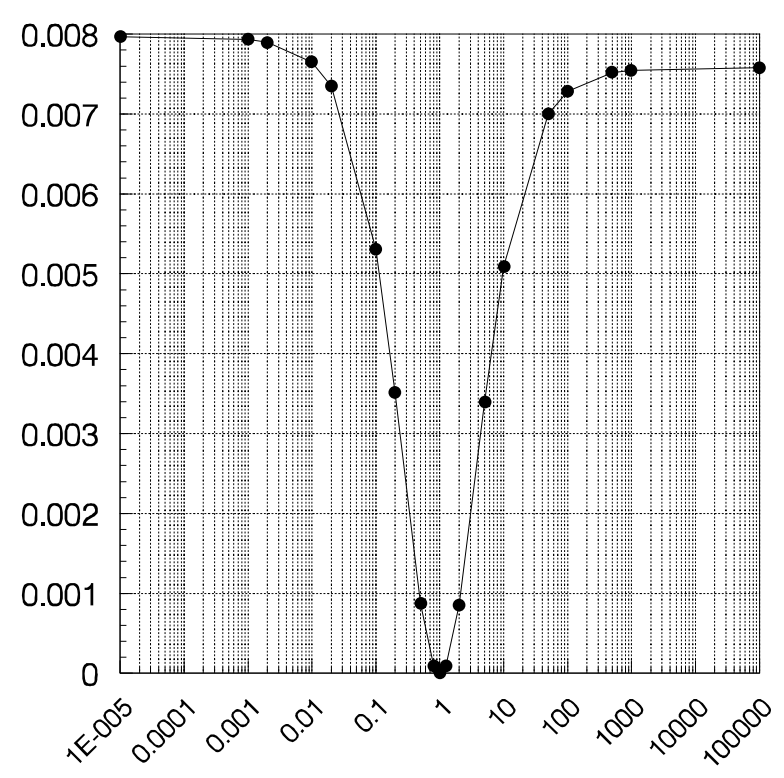

(b) $k$ vs. $\left\|L\left(u_{\epsilon}-U\right)-\Sigma\right\|_{\infty}$

Figure 4: The remainder terms as a function of the conductivity, k.

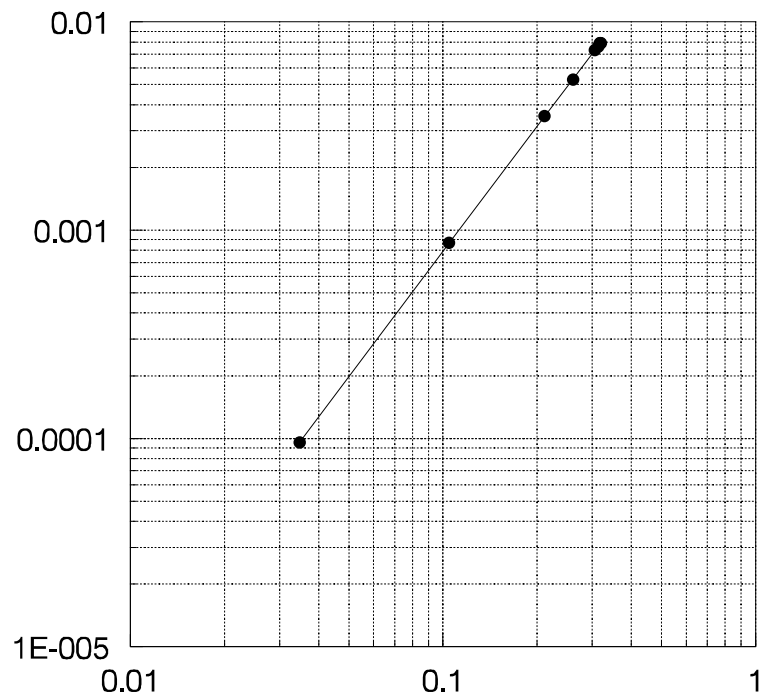

(a) $k<1$

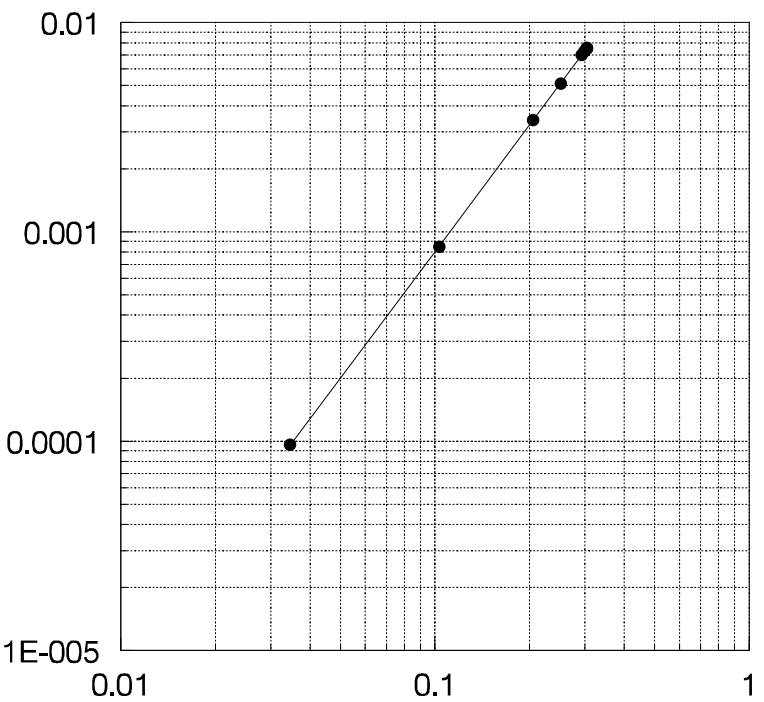

(b) $k>1$

Figure 5: Graph of $\left\|L\left(u_{\epsilon}-U\right)-\Sigma\right\|_{\infty}$ vs. $\left\|u_{\epsilon}-U\right\|_{\infty}$. 


\begin{tabular}{|c|r|c|c|}
\hline$z_{1} ; z_{2}$ & \multicolumn{1}{|c|}{$k$} & $u_{\epsilon}-U$ & $L\left(u_{\epsilon}-U\right)-\Sigma$ \\
\hline$(0,6) ;(0,-6)$ & 10 & 0.281 & $8.49 \mathrm{E}-3$ \\
\hline$(0,6) ;(0,3.9)$ & 10 & 0.360 & $7.14 \mathrm{E}-3$ \\
\hline$(0,6) ;(0,3.9)$ & 1000 & 0.425 & .100 \\
\hline$(6,0) ;(3.9,0)$ & 10 & 0.823 & .150 \\
\hline$(6,0) ;(3.9,0)$ & 1000 & 1.075 & .255 \\
\hline
\end{tabular}

Table 1: $L^{\infty}(\partial \Omega)$ norms of remainder terms

coefficient for $k<1$ to be 0.984 , and produces the "best fit", $t=0.0768 s^{1.99}$, where $t$ represents $\left\|L\left(u_{\epsilon}-U\right)-\Sigma\right\|_{L^{\infty}(\partial \Omega)}$ and $s$ represents $\left\|u_{\epsilon}-U\right\|_{L^{\infty}(\partial \Omega)}$. The correlation coefficient for $k>1$ is 0.984 , and the corresponding "best fit" is $t=0.0826 s^{2.01}$. These results clearly indicate that the remainder $L\left(u_{\epsilon}-U\right)-\Sigma$ is of the order of the square of the remainder $u_{\epsilon}-U$ itself, uniformly in $k$. This helps to explain why the polarization tensor term in (54) (or (55) has the "correct" limit as $k \rightarrow 0$ and $k \rightarrow \infty$.

In our last example to assess the accuracy of our representation formula we study the remainder terms $u_{\epsilon}-U$ and $L\left(u_{\epsilon}-U\right)-\Sigma$ as we vary the location (closeness) and common conductivity, $k$, of two circular inhomogeneities. The results are displayed in Table 1 . In the first case, the (unit) disks, located at $(0,6)$ and $(0,-6)$, are relatively far apart and the corresponding remainder $L\left(u_{\epsilon}-U\right)-\Sigma$ is quite small. In the remaining four cases the disks are only a distance 0.1 apart. When the two disks are centered on the $x_{2}$-axis and $k$ is moderately to very large they have less impact on the electric field (which largely flows parallel to the $x_{1}$-axis), than when they are centered along the $x_{1}$-axis. This explains why the remainders are smaller in cases 2 and 3 when compaired with cases 4 and 5 . When $k$ is 10 the remainder $L\left(u_{\epsilon}-U\right)-\Sigma$ is smaller than when $k$ is 1000 (for the same geometric configuration). In the second case when $k$ is 10 and the disks are close, but centered on the $x_{2}$-axis the remainder $L\left(u_{\epsilon}-U\right)-\Sigma$ is small enough (compared to $u_{\epsilon}-U$ ) that our approach would very easily locate these two disks, however, as a general rule we must conclude that our approach will be somewhat inaccurate when it comes to locating inhomogeneities that are closely spaced.

\subsection{Identification experiments}

As mentioned earlier our identification approach consists in solving the least squares problem (78). For the actual minimization we employ Moré's routine, a modified Levenberg-Marquardt algorithm. To be quite specific, we call the general least-squares solver LMDIF1 which is found in MINPACK. Note that we solve the unconstrained problem. That is, in solving (78) we do not place any restrictions on $z_{i}$ or $\epsilon \rho_{i}$. Although we could have imposed conditions requiring that for each $i, z_{i} \in \Omega$, and $\epsilon \rho_{i}$ be sufficiently small so that the inhomogeneity centered at $z_{i}$ remains strictly inside $\Omega$, these constraints were unnecessary. When the true number of inhomogeneities is known, these restrictions have always been 
automatically satisfied in the cases we have run. Moreover, when the number of inhomogeneities is a priori unknown, then the unconstrained problem can in our experience be used to determine the number of inhomogeneities as follows. If the number of inhomogeneities in the initial configuration is less than the actual number present (in the data), then the final $l^{2}$ residual produced remains relatively large. Upon increasing the number of initial inhomogeneities, this residual decreases until the number of inhomogeneities corresponds to the actual number present. If the initial number of inhomogeneities is further increased, the additional inhomogeneities were eventually placed outside the domain $\Omega$ or shrunk to zero. This provides an excellent indicator that one has exceeded the actual number present.

When it comes to the requirement that the inhomogeneities stay disjoint and well separated our intermediate iterates are frequently in violation. Admitting overlapping inhomogeneities has in practice proven extremely useful in improving the convergence of our procedure and in providing a simple (and quite reasonable) initial estimate for the location and the size. The initial inhomogeneities are all placed at the center of the domain. A "rough" estimate for the "size" of the inhomogeneities is obtained by considering (78) with a prescribed number of inhomogeneities (all "centered" at the origin and of identical "size") and then solving for $\epsilon \rho_{i}=\epsilon \rho$.

If the common scale $(\epsilon)$ of the inhomogeneities is extremely small, then our discretization errors may well dominate the data $u_{\epsilon}-\left.U\right|_{\partial \Omega}$, and therefore, one can not expect to locate such inhomogeneities with good accuracy. Consequently, in all our computational work we have considered only those values for $\epsilon \rho_{i}$ satisfying $\epsilon \rho_{i}>0.10, \quad i=1, \ldots m$.

Our first experiment involves determining the location, size, and angle of rotation of an elliptical inhomogeneity which is parametrically defined by

$$
\begin{aligned}
& x_{1}=0+0.25\left(\cos (t) \cos \left(\frac{\pi}{4}\right)-2 \sin (t) \sin \left(\frac{\pi}{4}\right)\right) \\
& x_{2}=6+0.25\left(\cos (t) \sin \left(\frac{\pi}{4}\right)+2 \sin (t) \cos \left(\frac{\pi}{4}\right)\right),
\end{aligned}
$$

and has conductivity $k=10$. Figure 6 depicts the convergence of the iterates obtained from the least squares routine. The initial guess and all the iterations have been graphed. After three iterations the location, size, and rotation of the inhomogeneity were acurately determined to three significant figures. The final result corresponds to the solid line ellipse.

We have (understandably) not been very successful in computationally determining the location, size, and angle of rotation for multiple inhomogeneities. However, if the sizes of the inhomogeneities are known then we have been able to determine their locations and their angles of rotation. Our next experiment addresses such a problem. That is, we determine the locations and angles of rotation of the three ellipses which are parametrically given by 


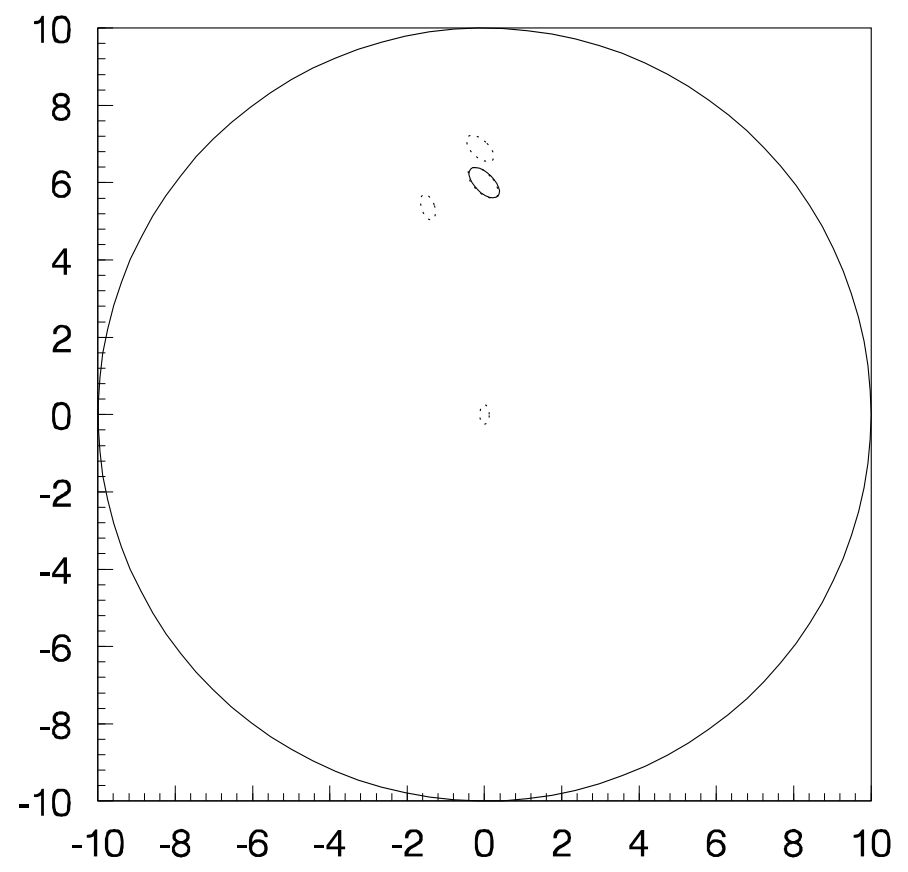

Figure 6: Graph of the iterations for an ellipse.

(a) $\quad x_{1}=7+0.25 \cos (t)$

(b) $\quad x_{1}=-1+0.50 \cos (t)$

$x_{2}=0+0.50 \sin (t)$

$x_{2}=-6+0.25 \sin (t)$,

(c) $\quad x_{1}=-2+0.25\left(\cos (t) \cos \left(\frac{\pi}{4}\right)-2 \sin (t) \sin \left(\frac{\pi}{4}\right)\right.$

$x_{2}=4+0.25\left(\cos (t) \sin \left(\frac{\pi}{4}\right)+2 \sin (t) \cos \left(\frac{\pi}{4}\right)\right.$.

For all three ellipses we utilize the same rescaled polarization tensor which is computed for the ellipse parametrically defined by $x_{1}=\cos (t), x_{2}=2 \sin (t)$. The program is able to accurately determine the locations, $z_{i}$, and the angles of rotation, $\theta_{i}$, for all the inhomogeneities by the fifteenth iteration. Each of the inhomogeneities has a (fixed) scaling factor of $\epsilon \rho=0.25$. For our initial data we center all the inhomogeneities at the origin with a zero rotation angle as shown in figure $7(\mathrm{a})$. The inhomogeneities, determined by the fifth, tenth, and fifteenth iterations of our program, are displayed in figure $7(\mathrm{~b})-(\mathrm{d})$ respectively. After the fifth iteration, two of the inhomogeneities were estimated to have approximately the same location and rotation. As a result, their images nearly coincide and only two inhomogeneities are visible in figure 7(b). Our computations yield the following locations and angles of rotation 


\begin{tabular}{|c|r|c|c|}
\hline Iteration & $z-$ Error & $\epsilon \rho-$ Error & Residual Norm \\
\hline 0 & 12.767145 & 0.385066 & $4.28148 \mathrm{E}-3$ \\
\hline 5 & 11.786228 & 0.346988 & $2.38138 \mathrm{E}-3$ \\
\hline 10 & 7.608637 & 0.308908 & $1.92556 \mathrm{E}-4$ \\
\hline 15 & 5.232940 & 0.180206 & $1.12363 \mathrm{E}-4$ \\
\hline 20 & 1.517874 & 0.124015 & $4.46768 \mathrm{E}-5$ \\
\hline 25 & 0.055479 & 0.005526 & $1.45346 \mathrm{E}-5$ \\
\hline
\end{tabular}

Table 2: Error analysis for iterations $0,5,10,15,20$ and 25

$$
\begin{aligned}
& \text { (a) } z_{1}=(6.982,-0.005), \quad \theta_{1}=3.134 \\
& \text { (b) } z_{2}=(-0.996,-5.976), \quad \theta_{2}=1.568 \\
& \text { (c) } z_{3}=(-2.026,4.024), \quad \theta_{3}=0.761
\end{aligned}
$$

In our third experiment we successfully locate and determine the sizes of five circular inhomogeneities. Each of the inhomogeneities has conductivity $k=15$. Figure 8 shows the initial estimate for the location and size of the inhomogeneities, as well as their estimated locations and sizes after $I=5,10,15,20$, and 25 iterations of the least squares algorithm. Table 2 provides an error analysis for the iterations. More specifically, column 2 represents the $l^{2}$ error in the location of the inhomogeneities after the I-th iteration. Column 3 represents the relative $l^{2}$ error in the size of the inhomogeneities after the I-th iteration, and column 4 represents the relative $l^{2}$ norm of the residual vector, that is,

$$
\text { Residual Norm }=\frac{\sqrt{\sum_{j=1}^{J}\left(L\left(u_{\epsilon}-U\right)\left(y_{j}\right)-\Sigma_{I}\left(y_{j}\right)\right)^{2}}}{\sqrt{\sum_{j=1}^{J}\left(u_{\epsilon}\left(y_{j}\right)\right)^{2}}} .
$$

Here $u_{\epsilon}$ is the (discrete) solution to the conductivity problem coresponding to the the "true" location of the inhomogeneities and $\Sigma_{I}$ is the sum from (78) with $z_{i}$ and $\epsilon \rho_{i}$ replaced by the approximate locations and scaling factors obtained after the $I$ th iteration.

For this example we have also calculated the relative $l^{2}$ norm of the boundary deviation $u_{\epsilon}-u_{\epsilon}^{F}$, where $u_{\epsilon}^{F}$ now is the (discrete) solution to the conductivity problem with inhomogeneities the locations and sizes of which are given by the final output from the minimization algorithm. This calculation yields

$$
\frac{\sqrt{\sum_{j=1}^{J}\left(u_{\epsilon}\left(y_{j}\right)-u_{\epsilon}^{F}\left(y_{j}\right)\right)^{2}}}{\sqrt{\sum_{j=1}^{J}\left(u_{\epsilon}\left(y_{j}\right)\right)^{2}}}=7.75533 \mathrm{E}-5 .
$$

As our last experiment we try to locate two circular inhomogeneities of a fixed radius $\epsilon \rho=.25$ as they approach one another. We consider five different 


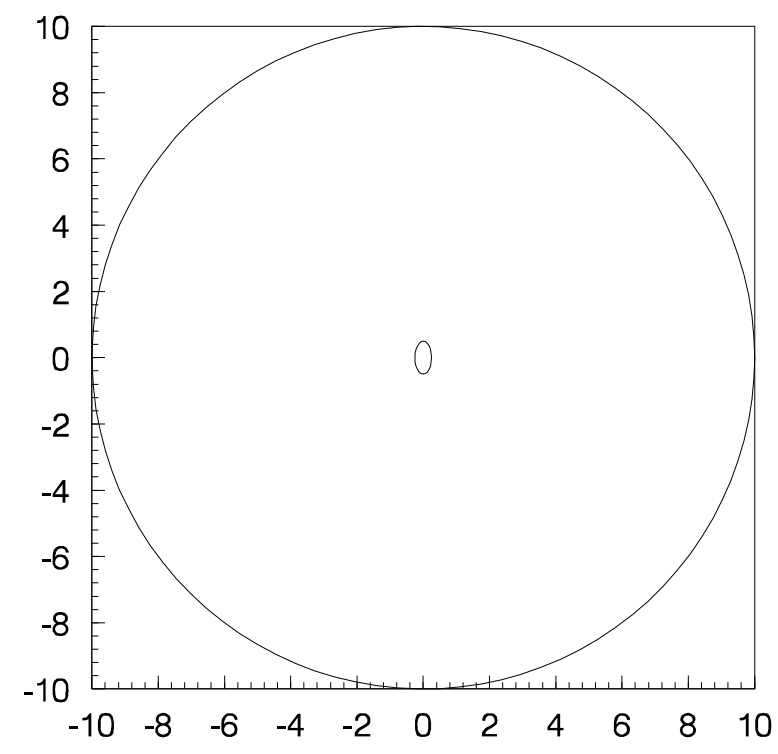

(a) Initial location and size.

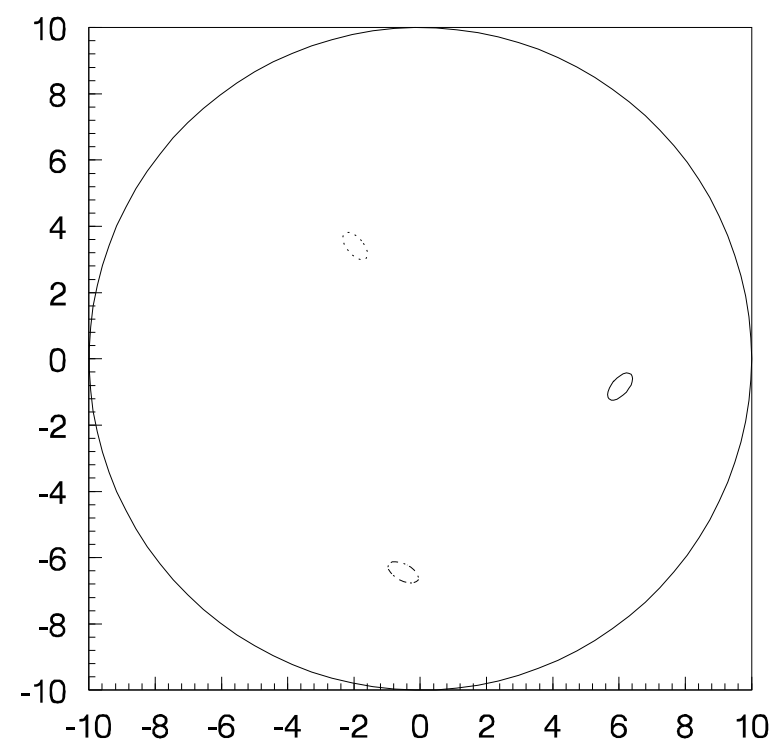

(c) Location and size after 10 iterations.

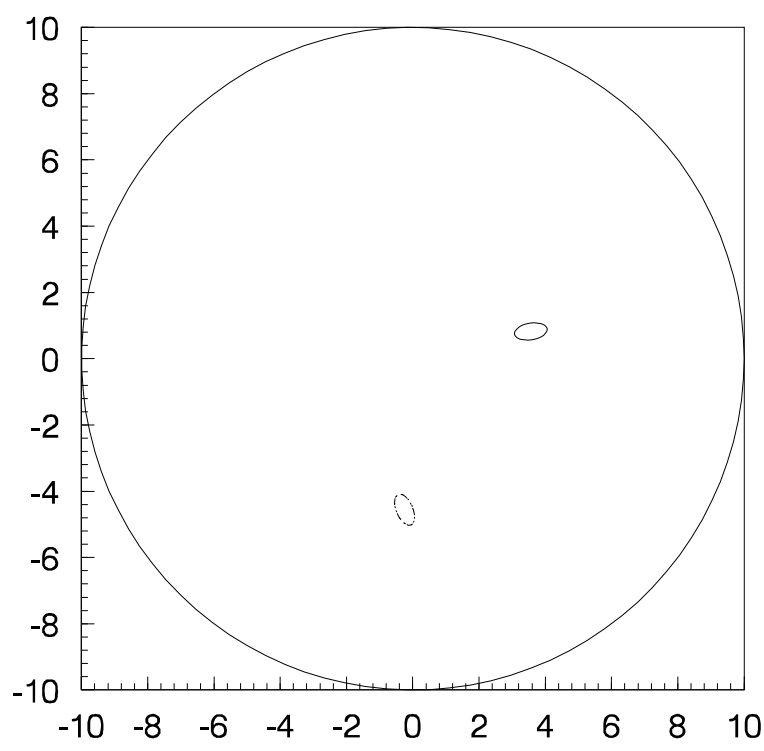

(b) Location and size after 5 iterations.

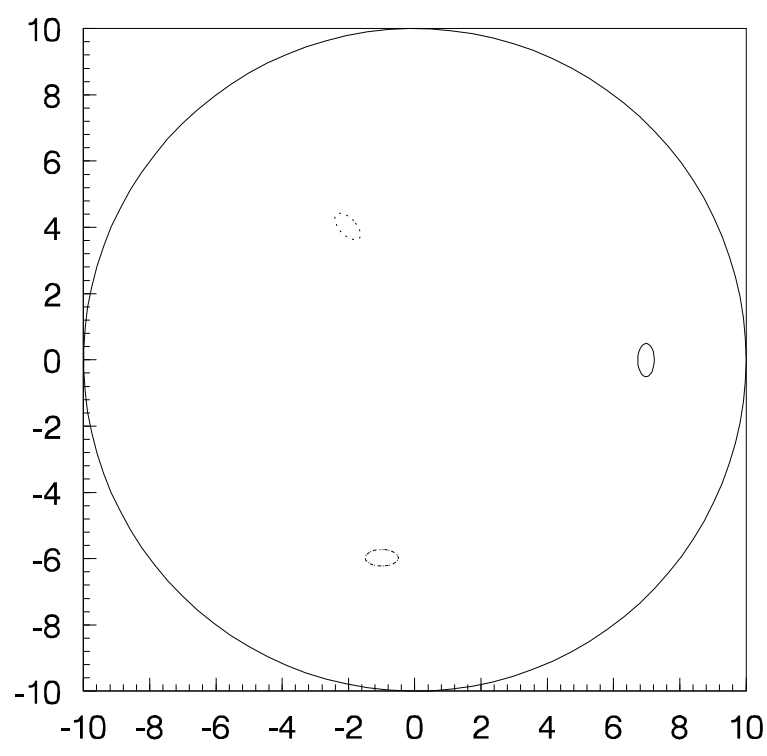

(d) Location and size after 15 iterations.

Figure 7: Convergence of iterates for 3 ellipses 


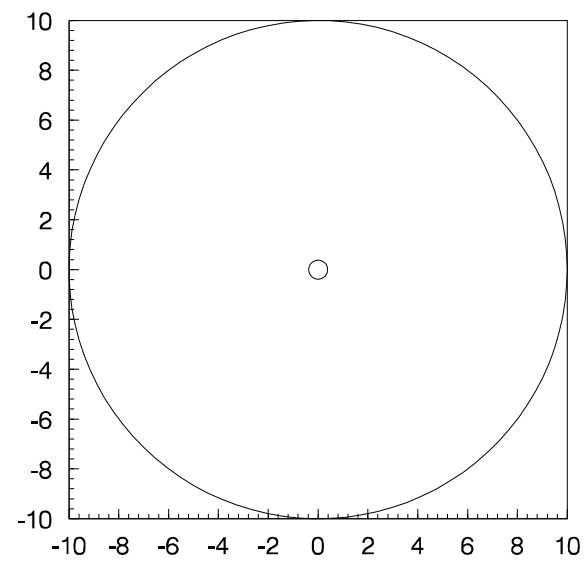

(a) Initial Guess: All inhomogeneities start at $(0,0)$ with an estimated radius of 0.38 .

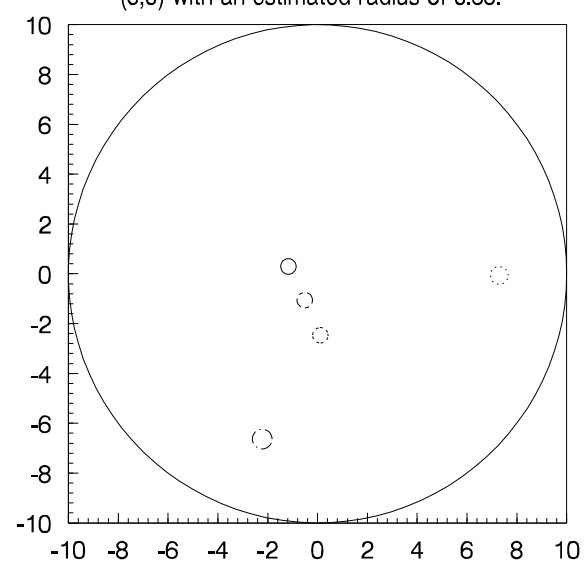

(c) Location and size of the inhomogeneities after 10 iterations.

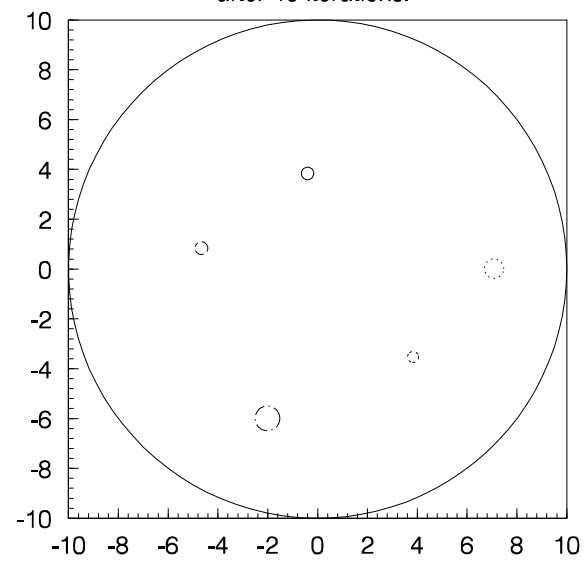

(e) Location and size of the inhomogeneities after 20 iterations.

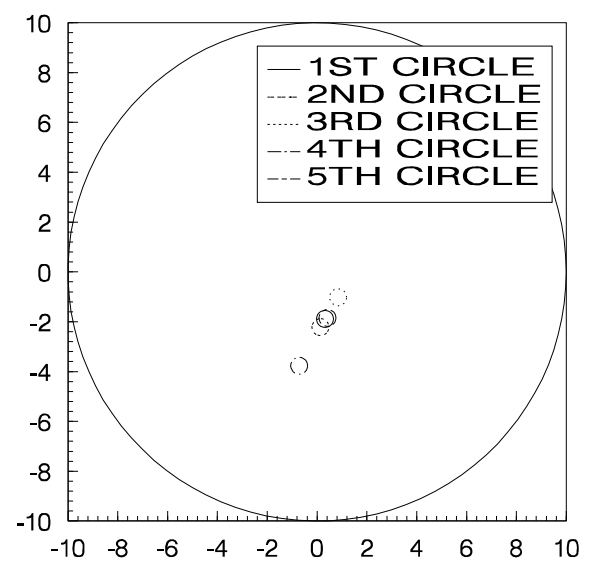

(b) Location and size of the inhomogeneities after 5 iterations.

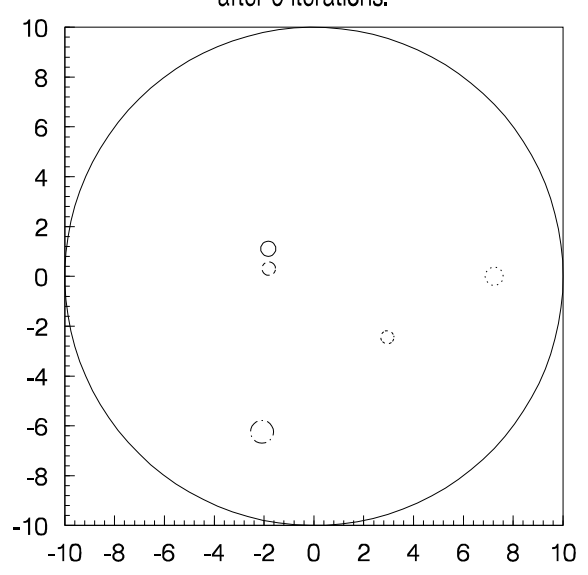

(d) Location and size of the inhomogeneities

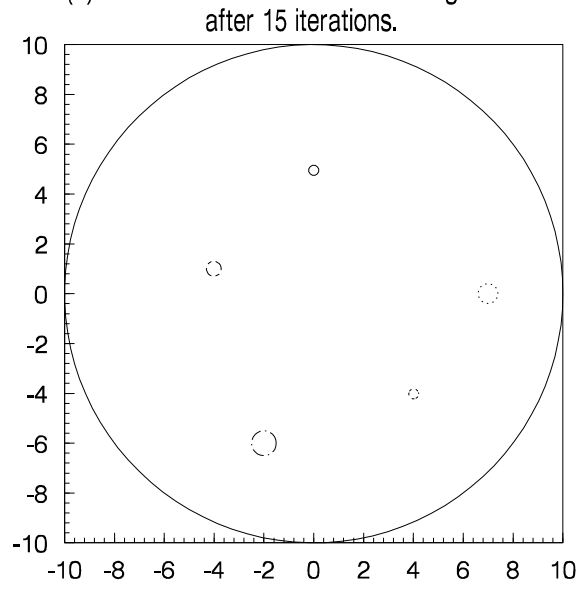

(f) Location and size of the inhomogeneities after 25 iterations.

Figure 8: Convergence of the iterates for 5 circular inhomogeneities. 
scenarios: the two circles are centered at $z_{1}=(-s, 0)$ and $z_{2}=(s, 0)$ with $s=$ $2,1, .75, .5$ and .3 corresponding to a distance between the two inhomogeneities of $3.5,1.5,1, .5$ and .1 respectively. For each scenario we run our minimization three times corresponding to three different initial guesses: (i) $z_{1}^{(\text {init })}=(0,0), z_{2}^{(\text {init })}=$ $(0,5)$, (ii) $z_{1}^{(\text {init })}=(0,-5), \quad z_{2}^{(\text {init })}=(0,5)$, and (iii) $z_{1}^{(\text {init })}=(-5,0), \quad z_{2}^{(\text {init })}=$ $(5,0)$. In all cases we start with initial scaling factors between 0.14 and 0.16 . In the first scenario $(s=2)$ our algorithm always succesfully determined the locations and sizes of the inhomogeneities. In the second scenario $(s=1)$ two of the runs were very succesful - the third got stuck near a local minimum but with a residual sufficiently large that it would indicate a problem (and thus warrant another run). In the third and fourth scenario the ability to identifty the two inhomogeneities accurately depended even more on the initial guess and quite troubling, the residual for a somewhat "inaccurate" identification was not always significantly larger than for a more "accurate" identification. Among other things this signals that the approximate formula on which our algorithm is based is starting to become "invalid". In the final scenario none of our initial guesses produced a very accurate identification of the inhomogeneities. In the best case the estimated locations and sizes of the inhomogeneities were $z_{1}=$ $(-0.280,0), \quad \epsilon \rho_{1}=0.274$ and $z_{2}=(0.292,0), \quad \epsilon \rho_{2}=0.269$.

We have tested our approach in a variety of other cases including those with multiple inhomogeneities having different shapes and different conductivities. Our experience in these cases is very similar to that described above. But we should note that for such problems the computational cost increases somewhat, since multiple polarization tensors are required.

\section{Acknowledgments}

This research was partially supported by NSF grant DMS-9704575 and AFOSR URI-RIP grant 93-NA206.

\section{References}

[1] Alessandrini, G., Isakov, V., and Powell, J., Local uniqueness in the inverse conductivity problem with one measurement, Trans. Amer. Math. Soc., 347 (1995), pp. 3031-3041.

[2] Barber, D., and Brown, B., Progress in electrical impedance tomography, pp. 151-164 in "Inverse Problems in Partial Differential Equations", D. Colton et. al. Eds., SIAM Publications, Philadelphia 1990.

[3] Cedio-Fengya, D.J., Ph.D. Thesis, Rutgers University, May 1997. 
[4] Cheney, M., Isaacson, D., Newell, J., Simske, S., and Goble, J., NOSER: An algorithm for solving the inverse conductivity problem, Int. J. Imaging Syst. and Technol., 22 (1990), pp. 66-75.

[5] Folland, G.B., Introduction to Partial Differential Equations, Princeton University Press, Princeton 1976.

[6] Friedman, A., and Isakov, V., On the uniqueness in the inverse conductivity problem with one measurement, Indiana Univ. Math. J., 38 (1989), pp 563579 .

[7] Friedman, A., and Vogelius, M., Identification of small inhomogeneities of extreme conductivity by boundary measurements: a theorem on continuous dependence, Arch. Rat. Mech. Anal., 105 (1989), pp. 299-326.

[8] Kang, H., and Seo, J.K., Layer potential theory for the inverse conductivity problem, Inverse Problems, 12 (1996), pp. 267-278.

[9] Kleinman, R.E., and Senior, T.B.A., Rayleigh scattering. Chap. 1 in, "Low and High Frequency Asymptotics", V.K. Varadan and V.V. Varadan, Eds., Elsevier Science Publishers, 1986.

[10] Kohn, R., and Vogelius, M., Determining conductivity by boundary measurements, Comm. Pure Appl. Math., 37 (1984), pp. 289-298, II. Interior results, Comm. Pure Appl. Math., 38 (1985), pp. 643-667.

[11] Kress, R., Linear Integral Equations, Springer-Verlag, Berlin 1989.

[12] Liepa, V., Santosa, F., and Vogelius, M., Crack determination from boundary measurements - reconstruction using experimental data, J. Nondestructive Evaluation, 12 (1993), pp. 163-174.

[13] Miller, K., Stabilized numerical analytic prolongation with poles, SIAM J. Appl. Math., 18 (1970), pp. 346-363.

[14] Nachman, A., Global uniqueness for a two-dimensional inverse boundary value problem. Annals of Math., 143 (1996), pp. 71-96.

[15] Nishimura, N., and Kobayashi, S., A boundary integral equation method for an inverse problem related to crack detection, Int. J. Num. Meth. Eng., 32 (1991), pp. 1371-1387.

[16] Schiffer, M., and Szëgo, G., Virtual mass and polarization, Trans. Amer. Math. Soc., 67 (1949), pp. 130-205.

[17] Sylvester, J., and Uhlmann, G., A global uniqueness theorem for an inverse boundary value problem, Annals of Math., 125 (1987), pp. 153-169. 